\title{
Guess-Work and Reasonings on Centennial Evolution of Surface Air Temperature in Russia. Part V: Stability Margin Towards Emergency
}

\author{
Yury Kolokolov* and Anna Monovskaya \\ Orel State University, Komsomolskaya 95, \\ Orel 302026, Russia \\ *2kolo@mail.ru
}

Received November 2, 2018

\begin{abstract}
The paper presents a discussion on an opinion about the stability margin towards an emergency in local climate dynamics from the bifurcation analysis viewpoint. With this purpose we propose to attract the practice-oriented bifurcation analysis, where the conflict-of-units between notions used to understand natural evolution processes and notions used to describe desirable artificial regimes is resolved by integrating analytics on the basis of modified bifurcation diagrams. The discussion focuses on the phenomenon of interannual temperature variability, where local annual maximums and minimums are analyzed with daily details in both time and temperature coordinates. This phenomenon is considered via the probable, periodical and regulator conceptions. Advantages of the regulator conception are verified by results of processing the data of temperature meteorological observations on daily means over the last 135 years. This conception is based on the HDS-hypothesis, in accordance to which local climate dynamics is determined by the natural competition between the amplitude quantization (restricted by the temperature Hysteresis) and time quantization (caused by the Double Synchronization). Thus an alternation between three elementary processes with the same period (year) and different patterns of annual warming-cooling cycles is supposed as a typical behavior for local climate systems, and the idea on high-dynamic local climate ensembles is developed instead of the conventional opinion on quasi-static local climate norms. Mechanisms of temperature changes due to abrupt shifts (so-called change-points) of the $H D S$-regulator parameters are distinguished from mechanisms of temperature changes due to bifurcations. The notion of a stability margin is used as a distance to an emergency and is visualized in the parametrical space. So, in spite of the mechanisms of temperature changes with/without bifurcations are different, their conflict-free sewing becomes conceptually possible in the context of the stability margin towards emergencies determined relatively bifurcation boundaries in the parametrical space. Since the discussed dynamics is not supposed to exist in terms of the traditional estimations concerning the observed local climate changes, then we believe that the paper would be interesting for scientists in the field of bifurcation analysis as well as for scientists and specialists, activity areas of which relate to the contemporary challenges connected with climate changes.
\end{abstract}

Keywords: Modified bifurcation diagrams; practice-oriented bifurcation analysis; conflict-ofunits; $H D S$-hypothesis; $H D S$-regulator; interannual variability; seasonality; stability margin; emergency forecasting; local climate change; risk assessment.

\footnotetext{
*Author for correspondence

This is an Open Access article published by World Scientific Publishing Company. It is distributed under the terms of the Creative Commons Attribution 4.0 (CC-BY) License. Further distribution of this work is permitted, provided the original work is properly cited.
} 


\section{Introduction}

At present, communication facilities and information technologies together with mass media provide visual illustrations to the realized weatherand-climate events which seem to be undesirable and dangerous to human life and activities. Global warming represents the incontestable fact; and the changes observed from the 1950th year are unexampled facts, including the increase in the number of episodes of extreme and abnormal weather events. At the same time, conclusions on changes in regional and global temperature trends in the future are quite varied. for example [Stocker et al., 2013: Kirtman et al.. 2013: Alexander \& Perkins, 2013; Westervelt et al., 2015; Tol, 2016; Hawkins et al., 2016; Kattsov, 2017; Camus et al., 2017; Klimenko et al., 2018; Sippel et al., 2018; Yin et al.. 2018: WMO-Bulletin 66. 2017: WMONo.1212, 2018; [IPCC Press release, 2018]. Objective scientific estimations on the rate of climate and ecological changes are very challenging; and results of such estimations can be behind the coming events, for example Grimm et al., 2013a; Grimm et al., 2013b; Gockede et al., 2017]. In particular, ten years ago, the scenario on thawing of Arctic ice during the first half of 21st century was considered like a speculative overstatement and an apocalypse prediction Bondur et al., 2009. This year, the North Sea route is already opened RT news, 2018], and the outlook for the future in the Arctic region is not so clear. Nevertheless, in spite of such discrepancies, the main question seems to be the following: what are the dominant reasons for the observed climate changes?

A climate system represents a nonlinear system, for example Rial et al., 2004; Scheffer et al., 2009; Thompson \& Sieber, 2011; Cimatoribus et al., 2014; Krauskopf \& Sieber, 2014; Oh et al., 2014; Williamson et al., 2016]. The absence or presence of qualitative changes (i.e. bifurcations) in nonlinear system dynamics can be based only on the results of the bifurcation analysis, which are usually visualized in the form of bifurcation diagrams. And observations (i.e. data of meteorological measurements) are the most reliable data to show the actual state of affairs, since experimentally-made bifurcation diagrams are inevitably more reliable than bifurcation diagrams built by model-based simulations. Next, not only significant but also minor change of a bifurcation parameter and/or minor disturbance can lead to a bifurcation; and, theoretically, it can be realized during a period. In other words, theoretically, a climate system can change qualitatively its behavior during one calendar year. Usually, a bifurcation leads to considerable changes; however, there are bifurcations accompanied by comparatively minor quantitative changes without a change of periodicity. At the same time, quantitative characteristics of a behavior can change considerably with parametrical variation only (i.e. without a bifurcation). Thus, to choose soundly the dominant reasons, it seems to be necessary to understand whether the observed climate changes are accompanied by bifurcations?

However, the bifurcation analysis is practically ignored in traditional methods to estimate local, regional and global climate dynamics, for example Kirtman et al., 2013; Blunden \& Arndt, 2016; Kattsov, 2017; WMO-No.1212, 2018; Yakovenko, 2018]. Attempts to consider the bifurcation analysis in applications to various aspects of weather and climate dynamics remain comparatively complex, for example Eisenman \& Wettlaufer. 2009: Scheffer et al., 2009: Thompson \& Sieber. 2011: Cimatoribus et al., 2014; de Saedeleer et al., 2013; Dijkstra, 2013; Krauskopf \& Sieber, 2014; Oh et al., 2014; Kolokolov \& Monovskava, 2015a, 2015b, 2016a, 2016b; Williamson et al., 2016; Lucarini \& Bodai, 2017; Sooraksa \& Chen, 2018]. Practical applications of such research are quite restricted. We believe that, first of all, this situation relates to the general problems of the practice-oriented bifurcation analysis Kolokolov \& Monovskava, 2016b, 2016c, 2018]. Per se, it can be briefly formulated as shown in Fig. 1(a)(center). Let it be theoretically assumed that the observed strange behaviors relate to nonlinear evolutional scenarios [Fig. 1(a)(right)]. Then bifurcations determine a wide palette of bright qualitative changes; and nonlinear interrelations determine shades of this palette. Within habitual human thought, such terms are usually out of considerations due to everyday activities being mainly connected with systems (industrial, urban, social, natural ones and so on), states of which are quite stable. As a result, the practicing [Fig. 11(a)(left)] and scientific [Fig. 17(a)(right)] viewpoints are too far from each other; and the corresponding dissonance forms so-called conflict-of-units between the notions used to comprehend natural evolution scenarios (for example, evolution of local climate dynamics) and the notions used to describe desirable behaviors (for example, habitual temperature patterns of local climate). 


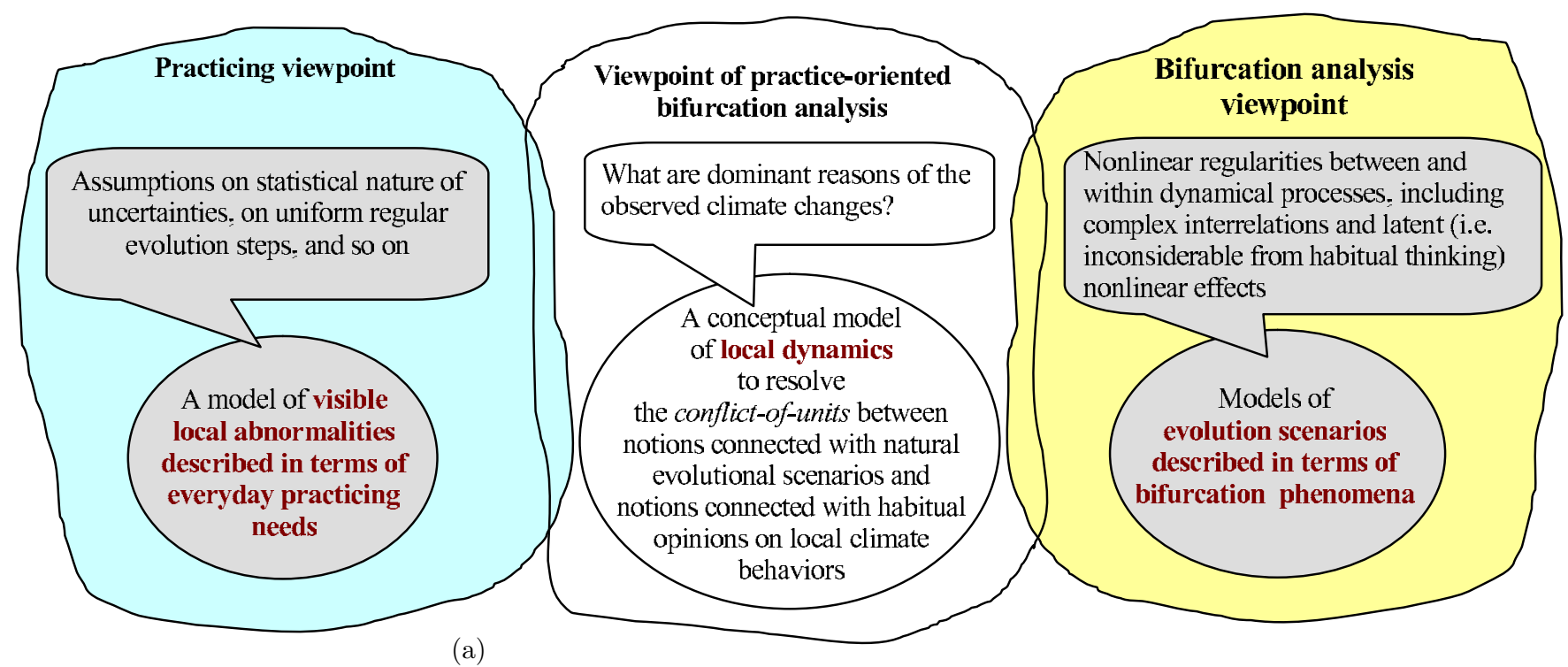

(b)

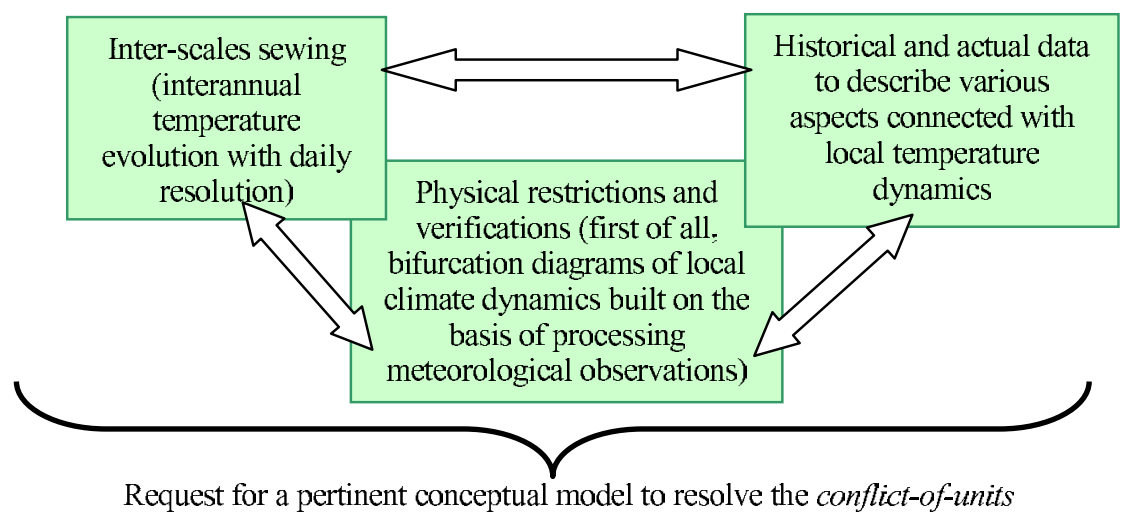

Fig. 1. (a) and (b) Schemes to the conflict-of-units in the application to interannual temperature variability observed in the local climate dynamics and the main demands concerning a pertinent conceptual model to resolve this conflict correspondingly.

Three main unresolved demands can be noted in this connection [Fig. 1(b)]. First, it is a point on how to sew weather and climate scales without distortions and losses of practically important information. From the actual state of affairs, for example Dutton et al., 2013; Buontempo et al., 2014; Glahn, 2014; Novak et al., 2014; Palmer, 2014; Soares \& Dessai, 2015: Trevisan \& Palatella. 2011: Uboldi \& Trevisan, 2015; : Rueda et al., 2017; Hourdin et al., 2017; Powers et al., 2017], desirable-to-practice time horizons of predictions (at least, 2-10 years) are usually considered for annual means averaged globally or regionally (a climate scale, upper horizontal part in Fig. 2); desirable-to-practice particularities of predictions (at least, local daily means) are restricted to 10-14 days (a weather scale, lower horizontal part in Fig. 2). However, the essential increase in information losses occurs from a weather scale to a climate scale through the sequential averaging from days to year (Fig. 2, left vertical arrow). Seasonal estimations within climate variability (central horizontal part in Fig. 2) try to approach these weather and climate positions. At present, regional estimations concern only monthly means with time horizons to six months long, for example Environment and natural resources, 2018; Hong Kong Observatorv. 2018: Hydrometeorological Center of Russia, 2018b; EFFIS, 2018]; and only few forecasts test either seasonal means up to decades in the future Klimenko et al., 2018] or monthly means up to 1 year in the future, for example NOAA, 2018]. Nevertheless, decadal time horizons and local details of daily means remain far from each other due to the actual time and space restrictions (Fig. 2, right vertical arrows). 


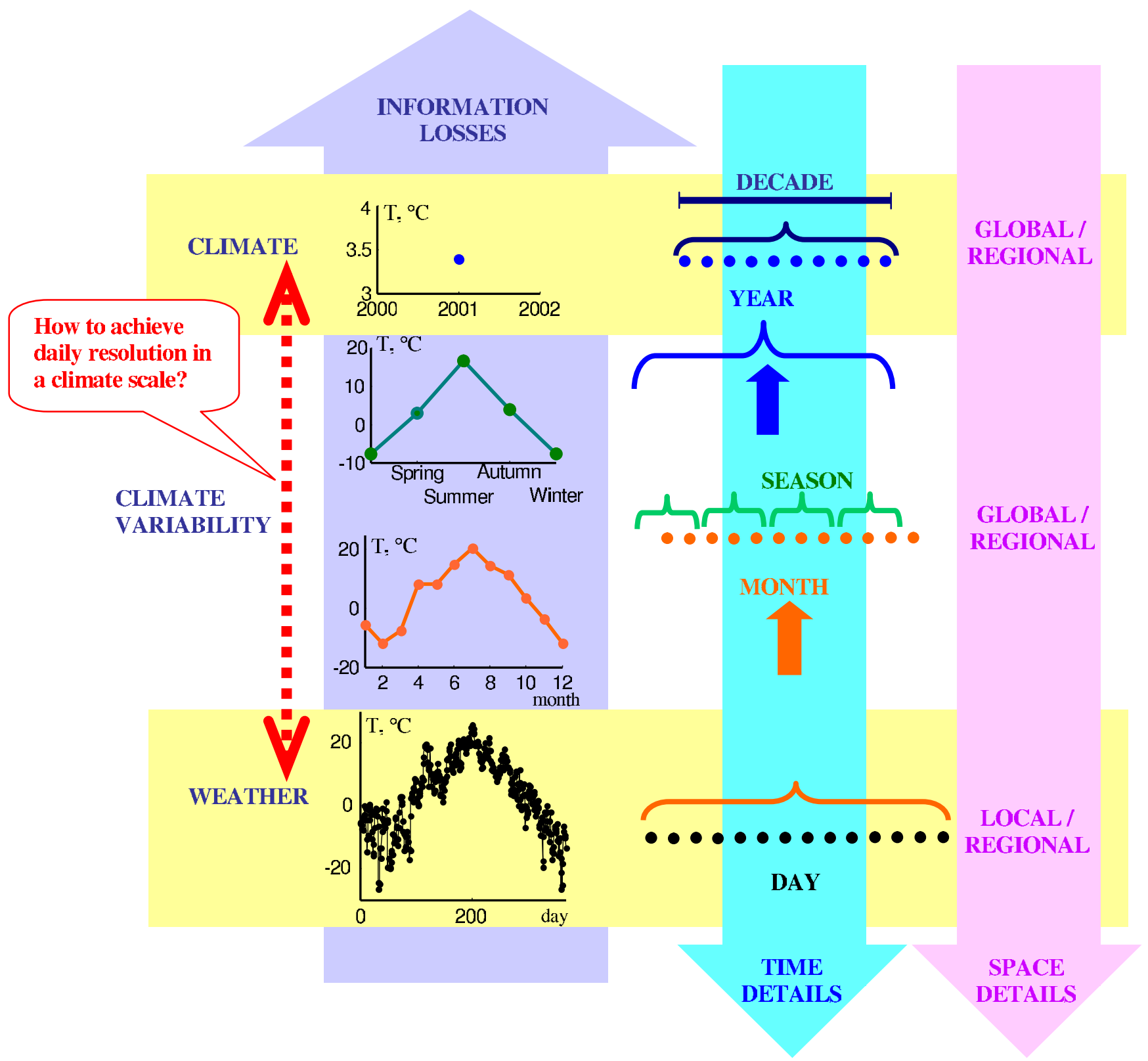

Fig. 2. Examples of the information losses over year through daily means — monthly means — seasonal means — annual means (left part) combined with the scheme of time and space details (right part).

The second and third demands [Fig. 1 (b)] are closely interrelated. Namely, the second one represents a point of physical restrictions and experimental verifications, with which a conceptual model is proposed. This point starts from the verification of the analyzable conceptual model by the observed phenomena. In particular, if the bifurcation analysis viewpoint is taken into account then a conceptual model should allow building bifurcation diagrams on the basis of processing the data of meteorological observations (i.e. building experimental bifurcation diagrams). The third demand represents a point of how to avoid the lack of the necessary data (meteorological, ecological, economic and so on) to research. In particular, it is necessary to take into account the following specific peculiarity connected with any local climate system: data on its dynamics are too slowly gathered and accumulated, and losses of these data are irreplaceable. The maximal horizon of the reliable meteorological observations is about 140 years (from year 1881); however, there are objective reasons, due to which local research can be done for the last 60 years only (from year 1961). Such horizon is very short, 
taking into account periods of climate oscillations (several decades and more). More long historical data or indirect data of meteorological reconstructions, for example de Saedeleer et al. 2013: Song et al., 2014; Sundqvist et al., 2014; Oh et al., 2014; Kose et al., 2017: Mavewski et al.. 2017: Margaritelli et al., 2018], provide usually too average values (monthly average, seasonal average, annual average, centennial average, and so on) or/and irregular values. These circumstances hold in the development of the experimental bifurcation analysis in applications to climate changes in a centennial scale.

We develop the hypothesis (so-called HDShypothesis) on local dynamics evolution, for example Kolokolov \& Monovskaya, 2015a, 2015b, 2015c, 2016a, 2016b, 2016d, 2017a, 2017b, 2017d, which satisfies all these demands somehow or the other. In accordance with the $H D S$-hypothesis, the local dynamics exists under the Hysteresis control with Double Synchronization ZZaytsev et al., 1975; Kolokolov et al., 1991]. The corresponding conceptual model (HDS-model) first provided the ability to build the bifurcation diagrams on the basis of processing the meteorological observations Kolokolov \& Monovskava, 2015a]. The discussion below starts from the classic axiomatic on local climate dynamics (Sec. 2) and moves on to the phenomenon of the interannual variability (Sec. 31). Next, the main positions of the HDShypothesis are briefly summarized (Sec. 4); and arguments to the HDS-hypothesis are discussed (Sec. 5). Next, cause-effect relations of temperature changes to show qualitative and quantitative mechanisms of the local dynamics evolution are introduced; and a novel view on the stability margin towards emergency is proposed from the practiceoriented bifurcation analysis (Sec. 6). The concluding discussion and future outlook are presented in Sec. 7. Discussion is accompanied by examples made by processing the meteorological data on the land surface air temperature daily means observed in Russia over the last 75-135 years. The data used in the paper originate from official open-access sites RIHMI-WDC. 2018a: Hydrometeorological Center of Russia, 2018a]. In spite of the paper being focused on the nonlinear dynamics viewpoint, we include interdisciplinary remarks in order to make the discussion interesting for specialists, activity areas of which relate to the contemporary challenges connected with climate changes.

\section{Problem Statement from the Uni-Behavior Axiomatic}

A climate system state is described by a vector of meteorological variables. Nevertheless, the temperature remains the main variable and indicator to analyze climate dvnamics Budvko. 1967: Stine \& Huybers, 2012; Kirtman et al., 2013; Stocker et al., 2013; Blunden \& Arndt, 2016; Hawkins et al., 2016; Hourdin et al. 2017: WMO-Bulletin 66. 2017: IPCC Press release, 2018]. It occurs due to various reasons, for example: the historical temperature data are generally the longest and the most detailed in time and space; the temperature measurements are the most reliable historical data; conceptual models describing temperature dynamics seem to be the most effective and illustrative; global consequences of climate changes are mainly estimated by relative temperature changes; and so on. We analyze historical observations on the land surface air temperature with daily resolution in time and local resolution in space. We believe that the temperature units described by these time and space terms provide fundamentally the ability to clarify doubtful and contradictory conclusions on climate dynamics mentioned in the context of the traditional climatology, for example Pezzulli et al., 2005: Argues \& Vose, 2011; Essex, 2011; Ruff \& Neelin, 2012: Gallanger et al., 2013; Huybers et al., 2014; Ji et al., 2014; Kim et al., 2015; Kolokolov \& Monovskava, 2015a, 2016b, 2016c: Sippel et al., 2015: Berner et al., 2017: Camus et al., 2017: Sevellec \& Drijfhout, 2018].

Let us concern several examples. At present, two conventional time windows to estimate climate norms are mainly used: years 1961-1990 and years 1981-2010, for example WMO-No.1137, 2014; Hydrometeorological Center of Russia, 2018c, 2018d. Other time windows are also used though, namely: years 1901-1950 Gruza \& Rankova, 2012], years 1880-1919 [Stocker et al., 2013], years 19862005 Kirtman et al., 2013], years 1850-1900 and 1880-1910 [WMO-Bulletin 66, 2017], and so on. Estimations based on the climate norms depend on the used time window, for example: local temperature evolution estimated relatively around years 1881-1910 [bold emerald line in Fig. 3(a)] shows the damped oscillation about the norm, but the one estimated relatively around years 1986-2005 [bold blue line in Fig. 3(a)] shows the increased oscillation towards the norm. And probable tendencies 
(a)
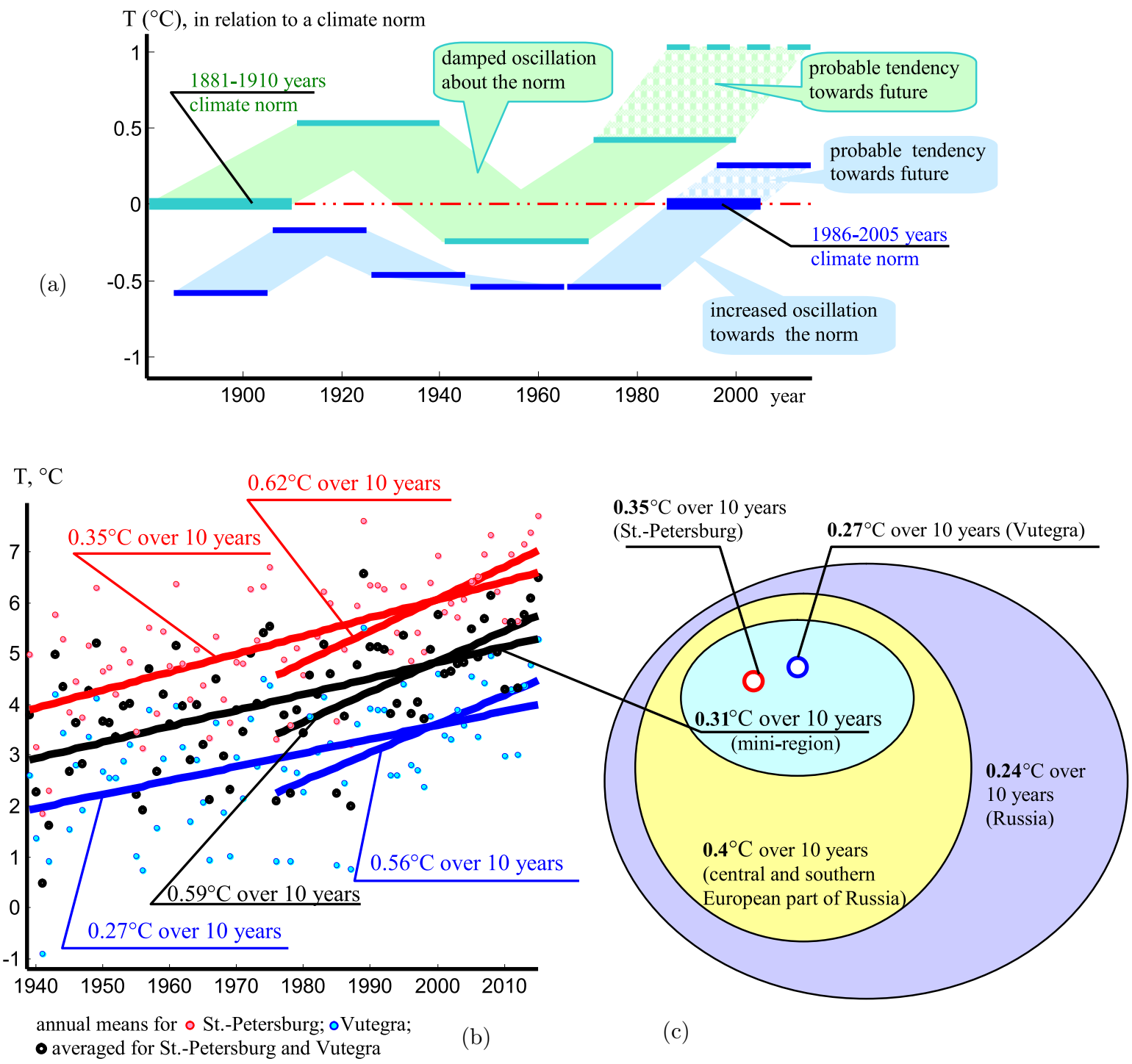

- averaged for St.-Petersburg and Vutegra

(c)

Fig. 3. (a) Steps of the temperature changes in Vutegra (WMO-code 22837) calculated in relation to the climate norms over years 1881-1910 (green bold horizontal line) and over years 1986-2005 (blue bold horizontal line), where the realized and probable steps are denoted by solid and dotted lines correspondingly. (b) Trend estimations for St. Petersburg (WMO-code 26063) and Vutegra (red and blue lines correspondingly) and their averaged tendency (as a mini-region) calculated over years 1976-2015 and years 1939-2015. (c) Variants of the traditional enlarged trend estimations over years 1939-2015, to which these local systems belong.

on the temperature increase towards the future are differently estimated. Since there is not a valid substantiation on which kind of such time windows is true, then a clear conclusion is absent.

Trend estimations on climate changes represent mainly linear coefficients built by annual means. The corresponding conclusions on local climate changes can be quite different due to them depending upon combinations of many factors (a prehistory, a space area, a composition of local climate systems, and so on). For example, official Russian reports consider traditionally the prehistory from year 1939 RIHMI-WDC, 2018b], though other beginnings are also used, namely: from years 1935 , 1936, 1976 [Blunden \& Arndt, 2016; Kattsov, 2017; Yakovenko, 2018], and so on. Two variants of the trend estimations for two quite close local systems (St. Petersburg and Vutegra) belonging to the Atlantic-continental forest north-west subregion Alisov, 1956] are presented in Fig. 3(b). However, such local details are not used and estimations are enlarged by various compositions, for example Bulvgina et al., 2015; Kattsov, 2017; Yakovenko, 2018; Klimenko et al., 2018; MGO, 2018]. As a result, 
typical uncertainties concerning local climate changes start at tens of percents [for example, Fig. 3(c)]. Since there is not a valid substantiation on which kind of such trend estimations is true, then a clear conclusion is absent.

Contemporary practical challenges suppose aspirations to the elaboration of climate estimations towards weather details and/or to prolongation of local details towards regional and global terms, for example Goddard et al., 2012; Dutton et al., 2013; Katz et al., 2013; Alexander \& Perkins, 2013; Palmer. 2014: Buontempo et al., 2014: Soares \& Dessai, 2015; Kolokolov \& Monovskava, 2016b, 2016c; Rueda et al., 2017; Hourdin et al., 2017; Sevellec \& Drijfhout, 2018; Sillmann et al., 2018]. From these challenges, it becomes necessary to reconsider space and time details for conceptual models. We believe that this situation is very important and needs further comments. The traditional conceptual model of annual temperature variation (hereafter $A T V$-model) remains the main unit to determine climate norms and tendencies as well as to analyze climate changes. The $A T V$ model is determined by local monthly means over 30 years [as illustrated in Figs. 4(a) 4(c)]. Here analytics relate to trivial operations from the statistical analysis (means and linear trends) concerning habitual notions (days, months, seasons, and years). However, strictly speaking, application of these operations to the mentioned notions without clear arguments can be questionable, for example Argues \& Vose, 2011; Essex, 2011; Ji et al., 2014; Kolokolov \& Monovskava, 2015a; Sippel et al., 2015], and there are dissonances in the traditional notions which are not originally intended to achieve the desirable-for-practice details throughout the desirable-for-practice terms Alexander \& Perkins, 2013; Kolokolov \& Monovskaya, 2016b, 2016c]. We believe that the main reasons relate to the used hypotheses on homogeneity of local dynamics in time and space scales. Let us illustrate it.

Let us consider regional dynamics with space details [Fig. 4(d)]. Actual ATV-models determined for each local climate systems over years 19611990 and over years 1981-2010 show three variants: the annual minimum can occur in January (J-form, black dotted subregion), or in February (F-form, red dotted subregion), or during JanuaryFebruary (JF-form, red-gray subregion). Then both the regional sampling consisting of January means and the regional sampling consisting of February means include heterogeneous elements (minimums and nonminimums); so, both averaging and trend analysis become questionable. Next, let us consider prehistory of the $A T V$-models over time from year 1901 for two local climate systems [Fig. 廿(e)]: FF-J-F-sequence occurs for St. Petersburg; JF-JFJ-J-sequence occurs for Vutegra. These sequences illustrate that even close local climate systems can evolve over time in different ways. So, a picture of quasi-homogeneous climate subregions can change over time. Let it occur due to a general climate change tendency within this region. However, why does one of the systems return to its previous kind [St. Petersburg, Fig. 4(e)] and the other one not return [Vutegra, Fig. 4(e)]? The ATV-models can, at least, indicate these facts. Subsequent averaging (seasonal means, annual means, and so on) disregards such information. It seems to be nonlogical simplification because any facts (ordinary as well as nonordinary ones) are important for a complete theoretical basis.

Let differences in trend estimations be excused by periodic constituents. For example, let us show sine-like functions: complete periodical waves demonstrate neutral trends [Fig. [5(a)], incomplete periodical waves lead to positive and negative trends [Figs. 5(b) and 5(c) correspondingly]. Here, the trend differences become more obvious with increase in pulsations [magenta solid lines in comparison with magenta dotted lines, Figs. 5(b) and $5(\mathrm{c})$ ] and with decrease in time terms [orange and magenta solid lines in Fig. [5 (b)]. So, let us compare temperature tendencies from year 1976 (over 40 years) and from year 1896 (over 120 years) in this manner for St. Petersburg and Vutegra [Figs. 5(d) and 5(e) correspondingly]. Thus various hypotheses appear to explain such trend differences: perhaps, it is a point of other period and/or other amplitude; perhaps, it is a point of variable periodicity and/or amplitude; perhaps, local climate systems are characterized by different periodicity and/or amplitude; and so on. So, which of such variants should be used in practical considerations for the future? Or else note: why are deviations from the trends essentially bigger before year 1989 [shaded by gray in Figs. [5(d) and $5(\mathrm{e})]$ than after?

Next, there are various assumptions on the nature of periodic climatic oscillations. For example, the Pacific Decadal Oscillation is the well-known climate index based upon patterns of the North Pacific surface temperature variation from 1900 to 
(a)
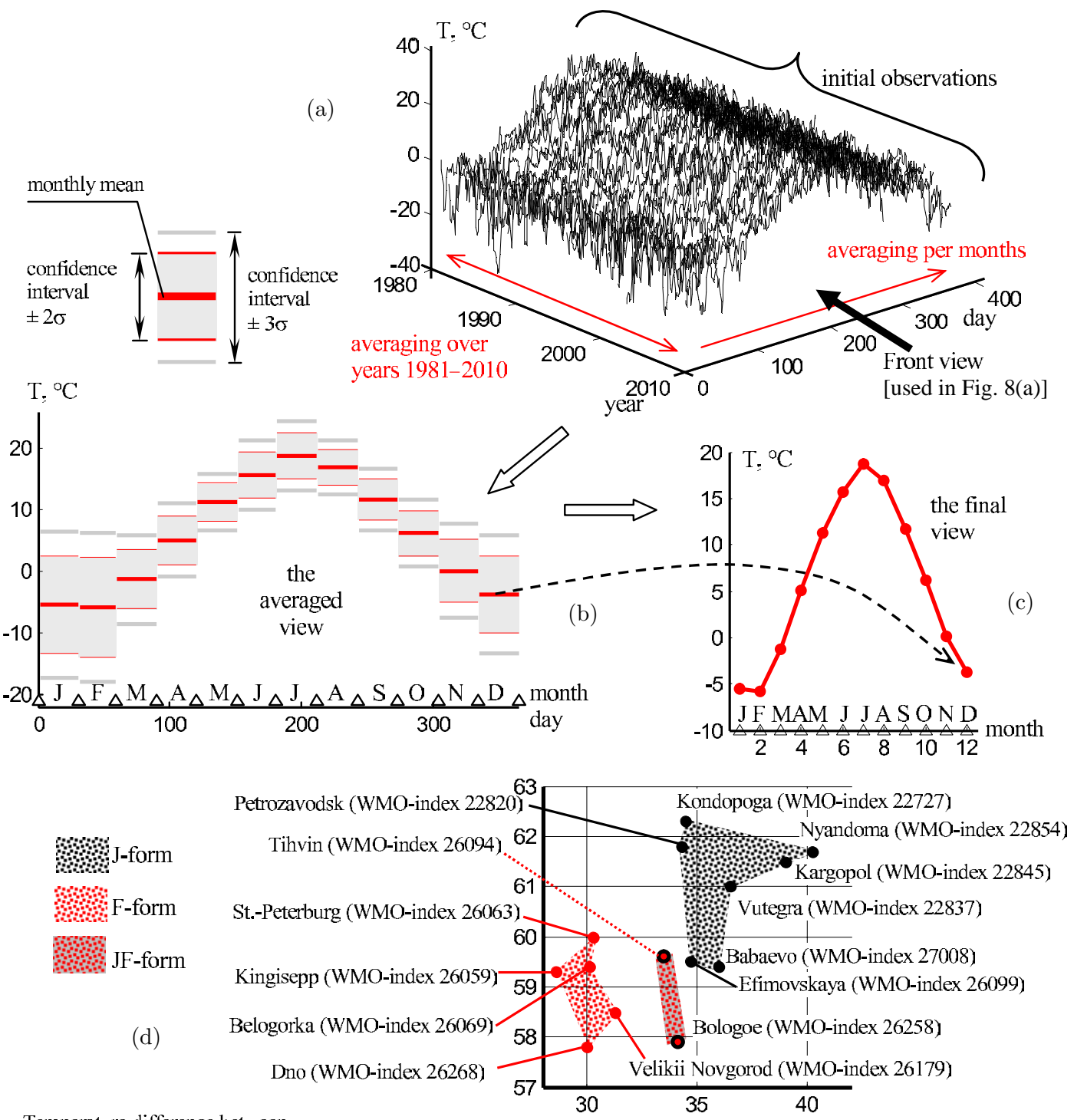

Temperature difference between
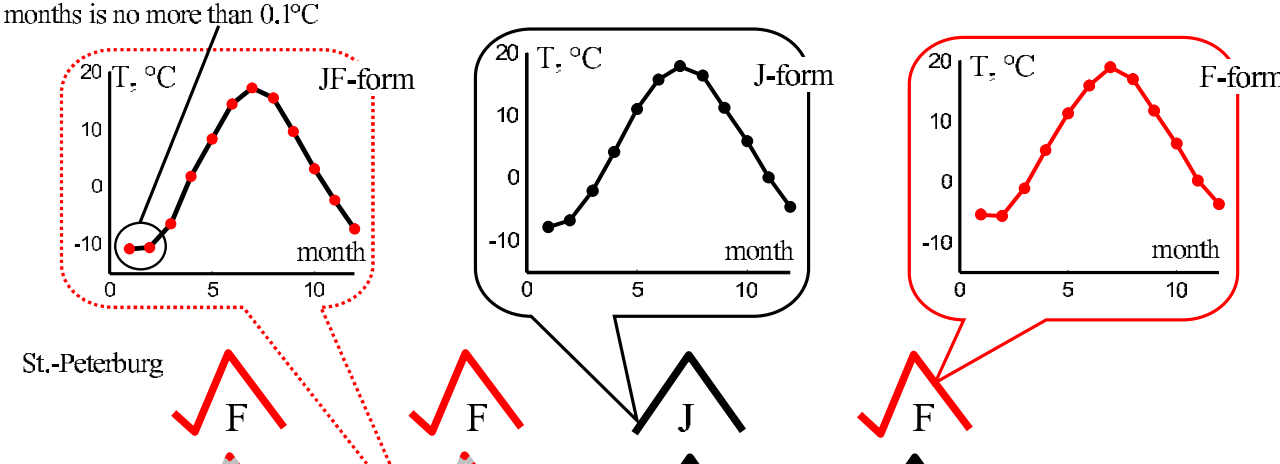

(e)

Vutegra
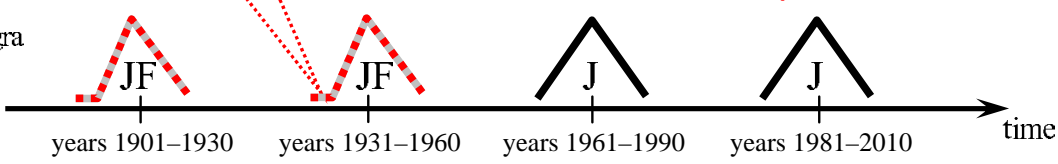

Fig. 4. (a)-(c) Initial temperature observations, averaging and the final view of the climate norm calculated for St. Petersburg. Illustrations concerning hypotheses on the homogeneity: (d) space diagram of distribution of three kinds of $A T V$-units over years 1981-2010 within a part of northwest subregion of Atlantic-continental forest region of the temperate zone (in accordance with classification by Alisov [1956]); (e) sequences of ATV-units over years 1901-2010 for St. Petersburg and Vutegra. 


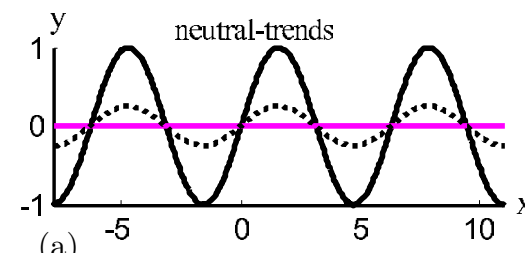

(a) -5

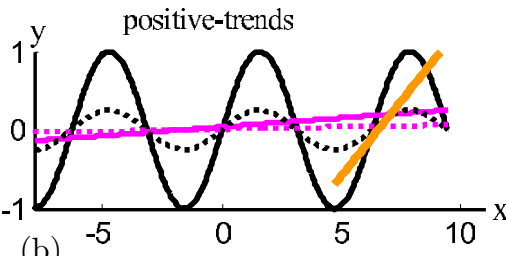

(b) -5

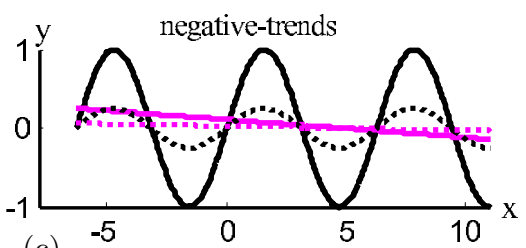

(c)

$0.17^{\circ} \mathrm{C}$ over 10 years $\quad 0.62^{\circ} \mathrm{C}$ over 10 years T. ${ }^{\circ} \mathrm{C} \quad$ (years 1896-2015) (years 1976-2015)
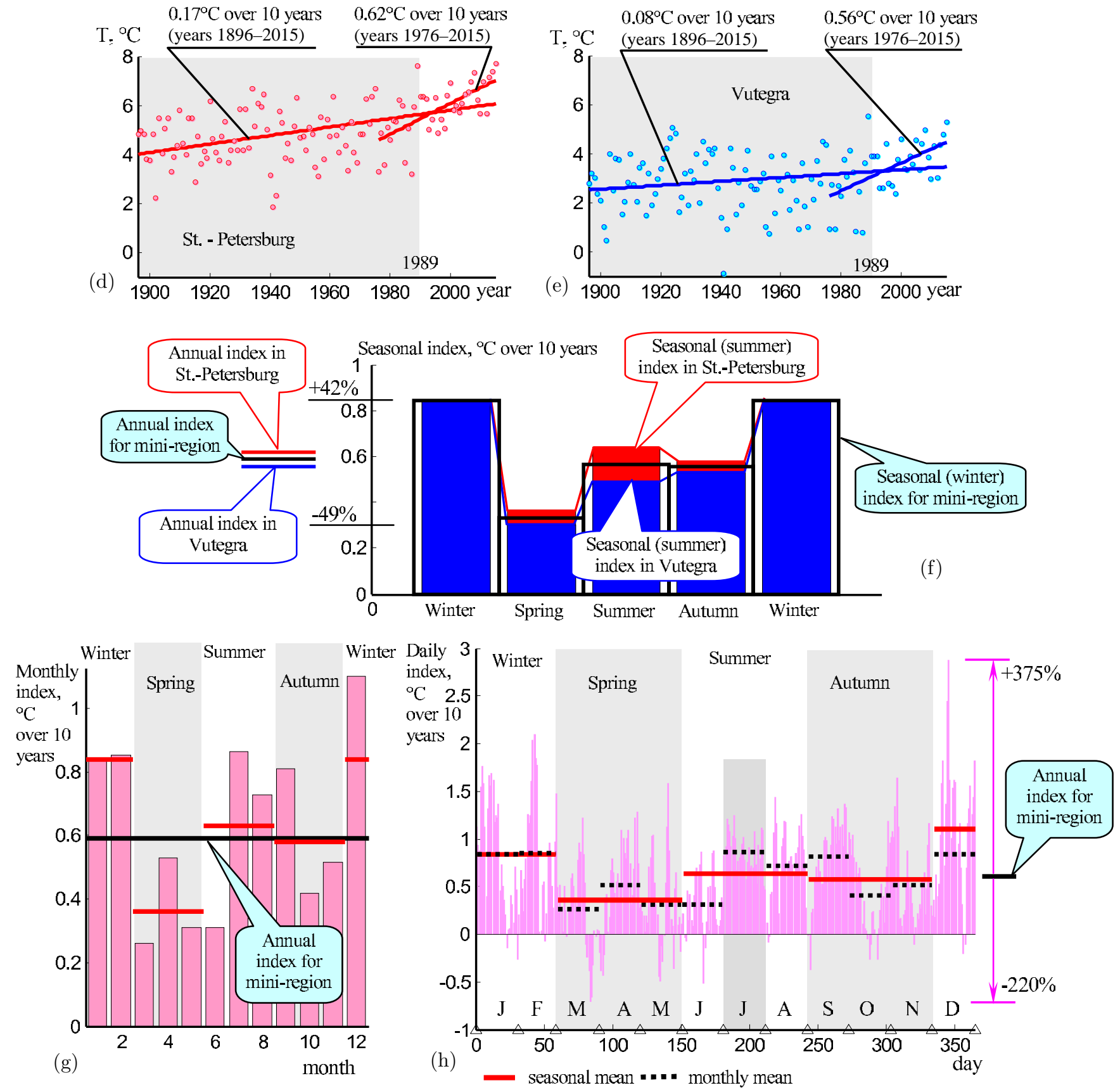

Fig. 5. (a)-(c) Trends for sine-like functions built for complete and incomplete periods. (d) and (e) Temperature tendencies built over 120 years and 40 years for St. Petersburg and Vutegra correspondingly. (f) Seasonal indexes in St. Petersburg (red bars) and Vutegra (blue bars) in comparison with the annual index within their mini-region (black contoured bars). (g) and (h) Interrelated monthly (g) and daily (h) indexes calculated for St. Petersburg. 
the present Mantua et al., 1997. The corresponding observations are well correlated with many records of North Pacific and Pacific Northwest climate and ecology. Warm and cold phases of this variation were approximately equal for about 20-30 years until 1998. However, these cycles were broken down recently [NOAA Fisheries, 2018]. In general, similar climatic oscillations represent approximate estimations and continue to be researched and discussed. In this context, it is a paradox that both monthly homogeneity and seasonal homogeneity used by traditional climate estimations are accepted as axioms. Yes, there are cases when an empirical assumption cannot be proved based on the existing scientific theories. However, in this case, high practical effectiveness could temporarily substitute a strict proof. So, let us discuss the abilities of the $A T V$-model conception from this viewpoint.

It follows the contemporary practical challenges on how to use climate generalizations to local weather details in order to satisfy everyday human activities. So, let us continue to consider years 19762015 , and let us trace the increase in uncertainty regarding the necessary details. The corresponding stages are presented in Figs. 5(f) $55(\mathrm{~h})$, where the averaged annual tendency for Vutegra and St. Petersburg (i.e. the annual tendency for a miniregion) is compared with annual-seasonal, seasonalmonthly, seasonal-monthly-daily tendencies correspondingly. Here, the notion of index denotes a linear coefficient calculated for the tendency during the corresponding time term (a season, month, day). The results of calculations demonstrate essential differences. For example, in the simplest case [Fig. [5(f)], the annual indexes are concordant with two seasonal indexes (in autumn and summer); however, deviations from other seasonal indexes amount to more than $40 \%(+42 \%$ in winter and $-49 \%$ in spring). Differences shoot up and achieve hundreds of percents for daily indexes [Fig. [5(h)]. At the same time, significant differences can occur not only for different time and space scales [Figs. [5(g) and [5(h)], but also for the same time and space scales [for example, deviations of the factual annual means from the tendency values in Figs. 5(d) and 5 (e)].

The phenomenon of interannual variability is the simplest particular case of the serious dissonances between large-scale and detailed temperature estimations, for example Pezzulli et al., 2005; Argues \& Vose, 2011; Essex, 2011; Stine \& Huvbers,
2012; Alexander \& Perkins, 2013; Ji et al., 2014; Kolokolov \& Monovskava, 2015b, 2015c, 2015d, 2016b; Sippel et al., 2015; Berner et al., 2017]. The interannual variability can be obviously identified twice per year (in winter and in summer), namely: temperature extremes are concentrated about two time locations in relation to conditional centerlines [denoted by red and blue points correspondingly, Fig. 6(a)]. It leads to the effects of the compressed and stretched fragments of annual warming-cooling cycles [bold solid and dotted black arrows in Fig. 6(a)] in comparison with the regular annual temperature variation in calendar terms [green arrows in Fig. 6(a)]. Such effects occur throughout the meteorological observations, for example: in the 19th century [Fig. 6(b)] as well as in the 21st century [Fig. G(c)]. In other words, the interannual variability seems to be a natural property of local climate dynamics.

In this connection, at present, two notions exist [Figs. 6(d) and 6(e)]: $A T V$-unit (from January 1, to the next one in accordance with the $A T V$-model) and $A W C C$-unit (from one temperature minimum to the next one) introduced in [Kolokolov \& Monovskaya, 2015a, 2015b, 2015d]. Correspondingly, $A T V$-units are described by equal duration but various forms [green sketches in Figs. 6(d) and 6(e)]; $A W C C$-units are described by various durations but equal triangle-like form [gray sketches in Figs. 6(d) and 6(e)]. Let dmin and dmax denote daily coordinate of annual minimum and maximum correspondingly [Fig. [6(f)]. Then, dmin- and dmaxtime series [Fig. 6(g)] demonstrate that great time dispersions of the temperature extremums [100 days and even more, Fig. [6(h)] are observed over the last 135 years, but not only for the last decades. So, the interannual variability seems to be a typical phenomenon with similar characteristics over the term of reliable meteorological observations at least.

Let us illustrate why does the description of local climate dynamics by $A W C C$-units seem to be important for practice? For example, let temperature minimums be in February in accordance with the actual climate norm [the climate norm in St. Petersburg over years 1981-2010, Fig. 4(e)]. So, let the corresponding preparation for the maintenance of an annual technological process be realized. However, in fact, temperature minimums (including extreme cold) can appear in December [Fig. 6(i)]. Yes, such facts are afterwards described; however, practical profits seem to be related to such 

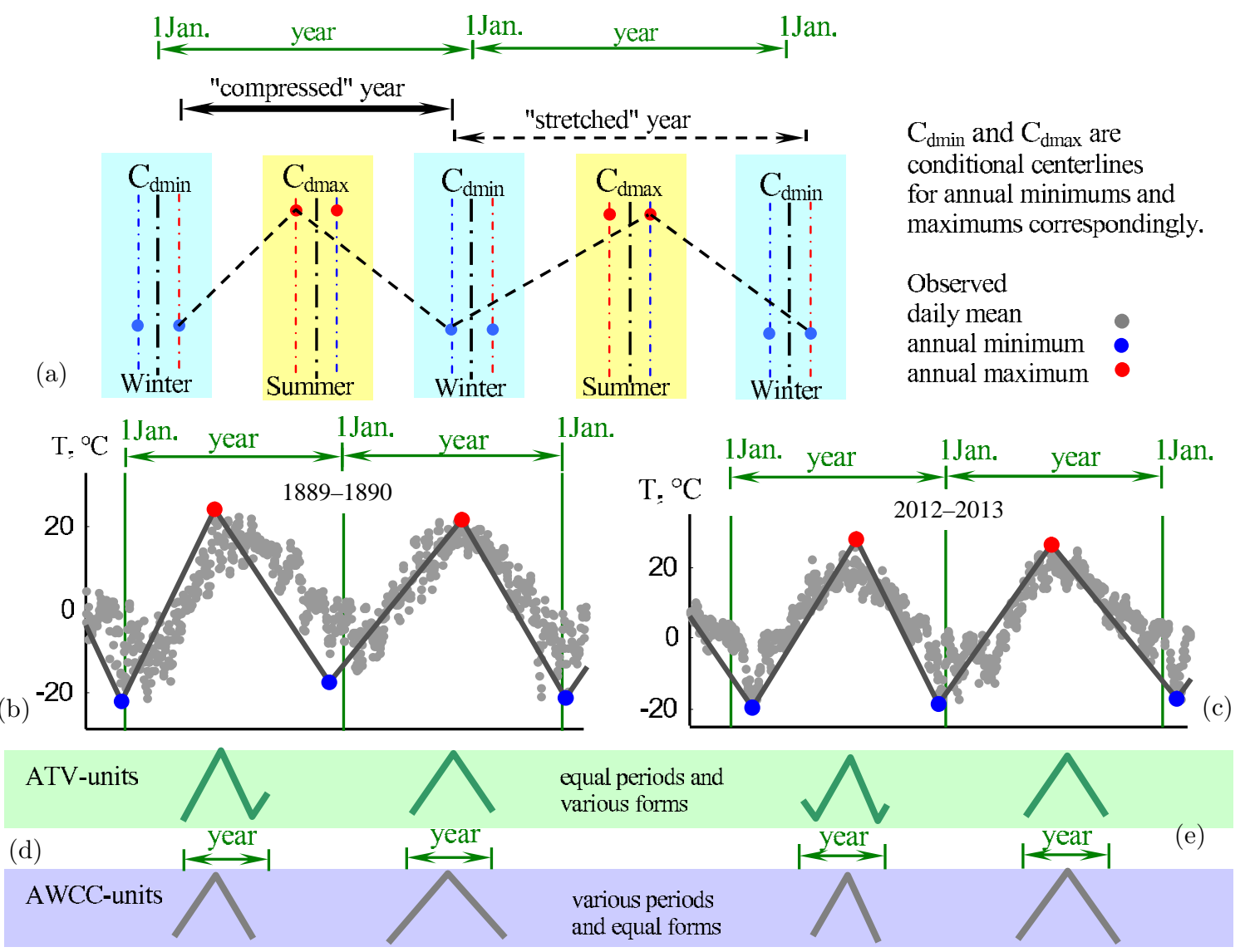

(f)

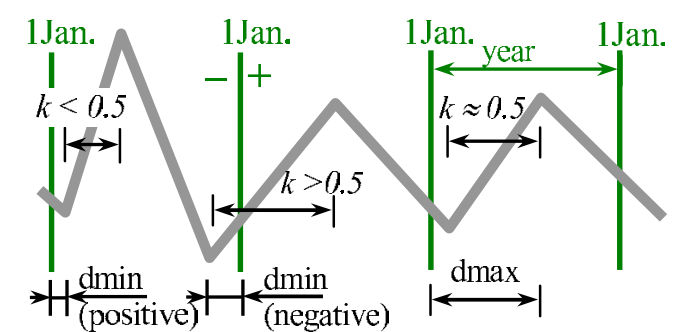

$(\mathrm{g})$
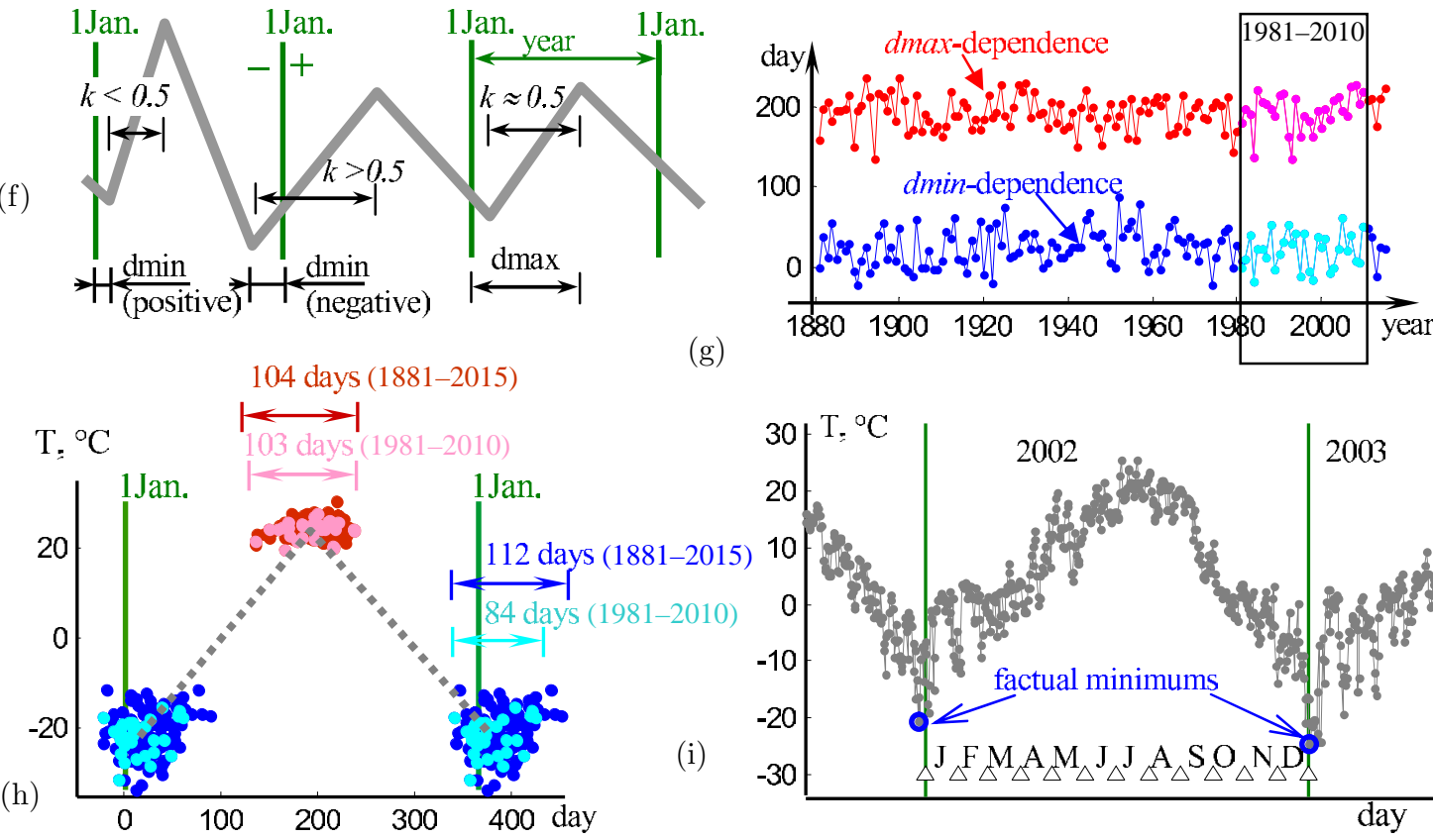

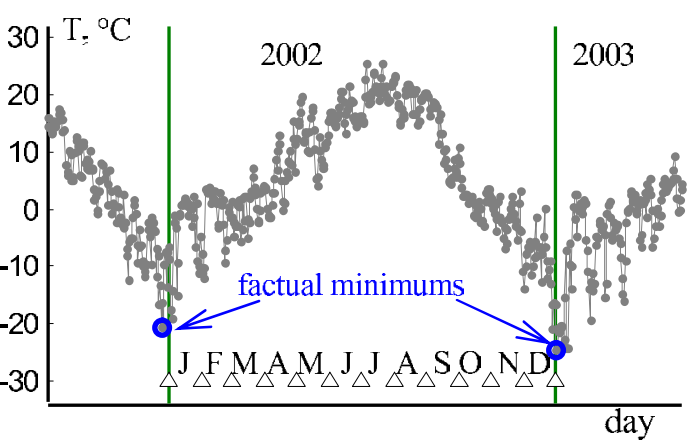

Fig. 6. (a) Effects of the interannual variability. (b) and (c) Fragments of the observed annual variation over years 18891890 and 2012-2013 correspondingly with (d), (e) comments to $A T V$-units and $A W C C$-units made for Figs. 6) b) and 6(c) correspondingly. (f) Coordinates to the interannual variability. (g) and (h) Sets of annual extremes (dmax- and $d m i n$-sets) shown over time by $d$ max- and dmin-dependences and shown within the limits of a calendar year correspondingly. (i) To the dissonance between the factual data and the climate norm over years 1981-2010. Results shown in Figs. 6(b), 6]c), 6)(g), 6(h) and $6($ i) are made by processing the temperature observations in St. Petersburg. 
comprehension which provides clear infallible forecasts. Since descriptions of the local dynamics from the $A T V$-model bring inevitable distortions, then these restrict the abilities of practice-oriented forecasts. We believe that progress in this field is connected with both unbiased detailed analysis and recomprehension of local climate dynamics from the bifurcation analysis viewpoint. The interannual variability seems to be one of the most evident tests which could verify the corresponding advances. And hereafter, we focus on the interannual variability in this sense.

\section{Towards Multibehavior Axiomatic}

Peculiarities of the local climate dynamics follow from physical properties which can be described by the corresponding conceptual model with combinations of internal and external parameters. At present, three conceptions are proposed to excuse the interannual variability. These conceptions can be conditionally denoted as probable, periodical and regulatory. The bifurcation analysis viewpoint supposes that a nonlinear system behavior can potentially change due to bifurcations, i.e. period or/and structure of a phase trajectory can change with variation of internal or/and external parameters. Then, the probable conception supposes that bifurcations are absent; the periodical conception supposes that periodicity can change; the regulator conception supposes that the period typically does not change but structures alternate. Let us discuss to what extent these conceptions provide the description of the observed events.

The probable conception describes the interannual variability by statistical deviations from an average, where the hypothesis on the normal distribution is accepted as the axiom and is widely used in the traditional estimations on climate norms and tendencies, for example Gruza \& Rankova, 2012; Kirtman et al., 2013; Bulvgina et al., 2015; Blunden \& Arndt, 2016; Kattsov, 2017; Yakovenko, 2018; WMO-No.1212, 2018]. Such estimations focus mainly on mathematical expectations (monthly means, seasonal means and so on, Fig. 2), in spite of many additional questions seem to be interesting to practitioners, for example [Fig. [4(b)]: expected deviations from each average (i.e. a standard deviation, $\sigma)$; enough confidence intervals $( \pm 2 \sigma$, or $\pm 3 \sigma$, or others); a number of misses beyond the accepted confidence; and so on. Since a statistical characteristic relates to a sampling, then a climate norm represents a notion related to the corresponding time window. Thus various uncertainties can appear. For example, in accordance with the official climate norms calculated over 30-year windows [Fig. 4(e)], two different locations of temperature minimums are expected for St. Petersburg during 1981-1990 and it can be formally presented by month(year)diagram [Fig. 7(a)]. At the same time, the realized events are more various and intricate than the events expected by the norms. For example, temperature maximums should always occur in July in accordance with the climate norms [Fig. 4(e)]; however, factually realized temperature maximums coincide with these expectations only in $51 \%$ of cases [red bars in Fig. Z(b)].

Generally, such differences can be shown by a month(year)-diagram built for 30-years windows sliding in one year [Fig. 7 (c), blue and red bold lines] in comparison with the factual realizations of temperature extremums [Fig. 7(c), blue and red circled points connected by thin lines]. Here, the expected and factual locations of extremes coincide in 38 combinations ( $28 \%$ over 135 years), where two expected locations of temperature minimums are taken into account in 34 cases among these 38 combinations. Discords between supposed and factual events occur throughout the range of reliable meteorological observations, [see for example Figs. [7(d) and [7(e)]. Statistical discords, contradictions and misses in climatology, for example [Pezzulli et al., 2005; Argues \& Vose, 2011; Essex, 2011; Alexander \& Perkins, 2013; Ji et al., 2014; Kolokolov \& Monovskaya, 2015a, 2016b, 2017c; Sippel et al., 2015], lead to rightful questions concerning the conclusions which are not based on the necessary confirmations (in particular, proofs concerning the hypothesis on the normal distribution). Results of studies concerning such aspects can be very constructive in order to advance comprehension of phenomena which cannot be explained until now or to resolve contradictions at least partly, for example Kolokolov \& Monovskava, 2015a, 2015c, 2016b, 2016c, 2017a, 2017b, 2017c; Kim et al., 2015; Berner et al.. 2017: Camus et al.. 2017: Sevellec \& Drijfhout, 2018].

So, let us concern statistical misses for factual temperature extremums. Let us suppose that the statistical misses occur due to jumps in temperature extremums. Let us continue to analyze the example 
the expected location of temperature minimums in accordance with the climate norm over years 1961-1990

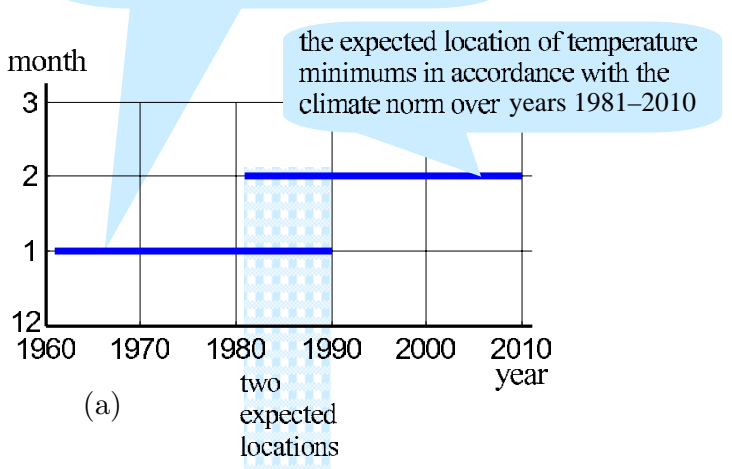

number, $\%$ over years $1881-2015$

locations expected by climate norms

(b)

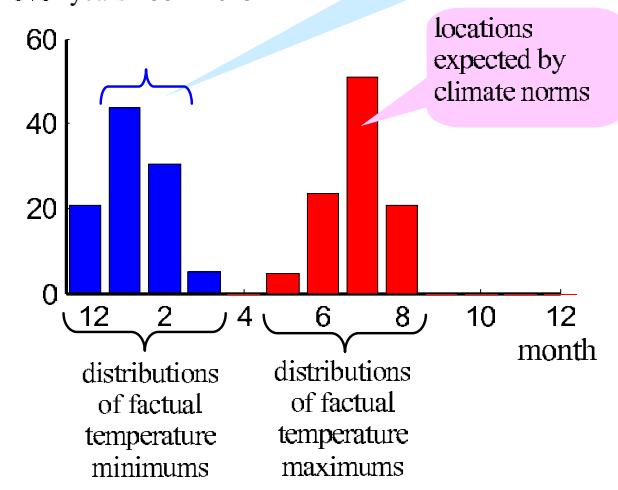

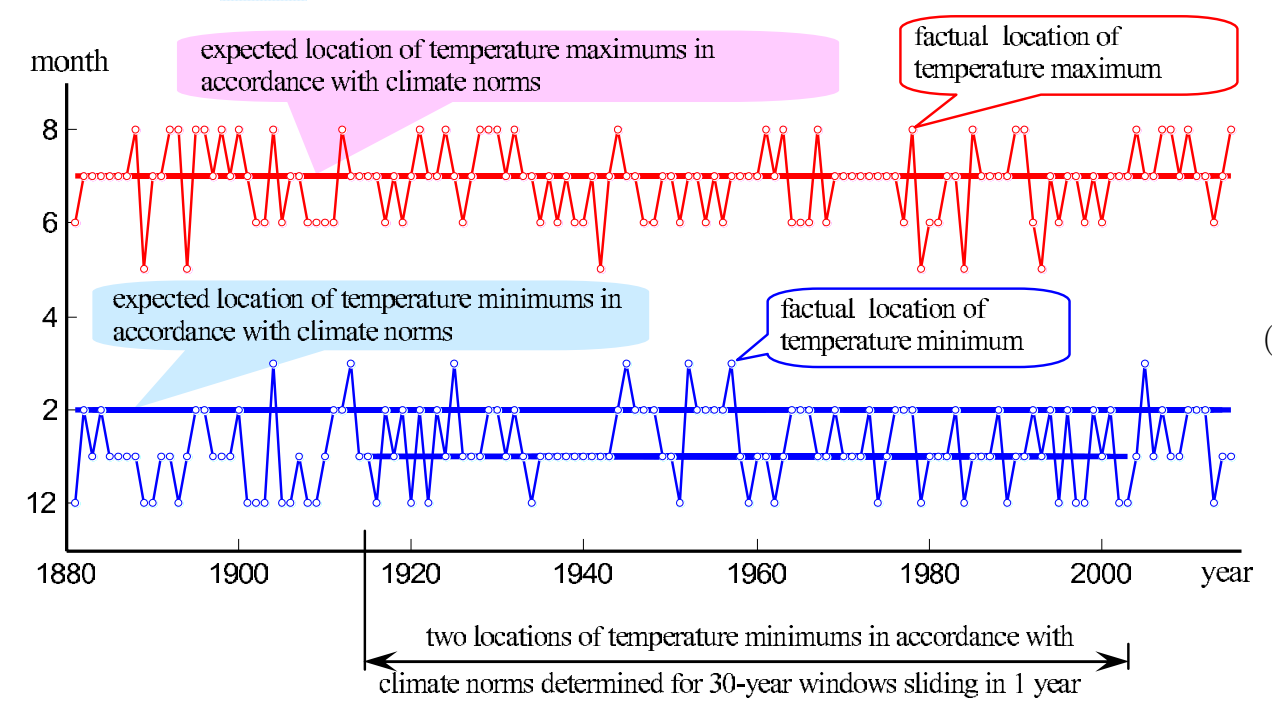
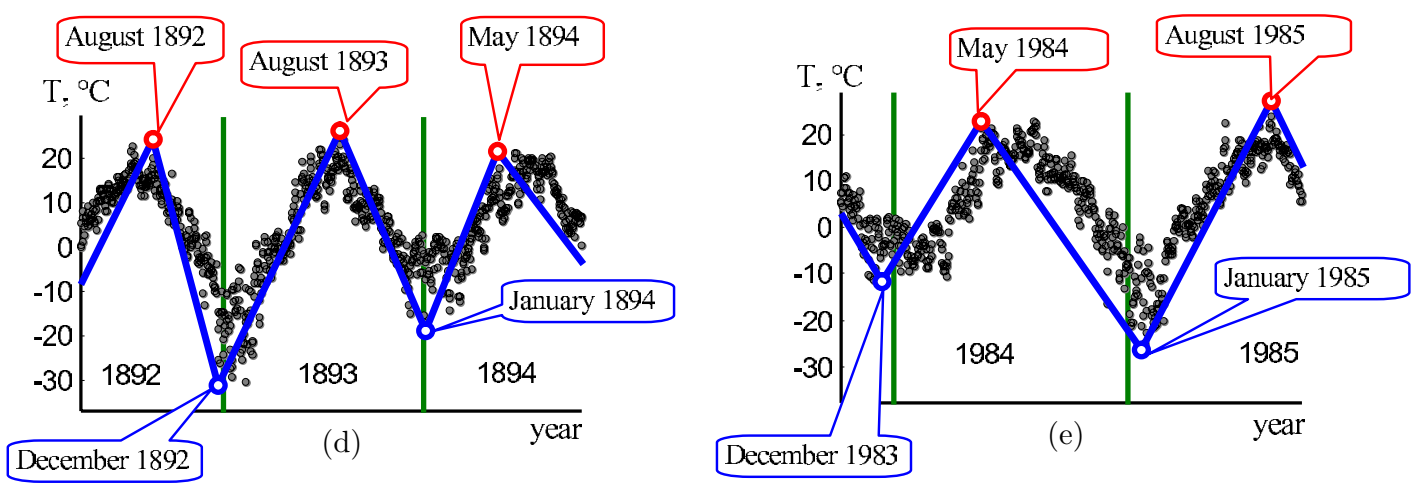

Fig. 7. Results of processing the observations made in St. Petersburg: (a) expected locations of temperature minimums determined for 30-year climate norms sliding in 20 years; (b) combining distributions (\%) determined for factual locations of temperature minimums (blue bars) and maximums (red bars) over years 1881-2015; (c) time diagrams to combine the expected locations of temperature minimums (blue bold lines) and maximums (red bold line) with the factual locations of temperature minimums (blue circled points) and maximums (red circled points) over years 1881-2015; (d) and (e) illustrations to unexpected locations of minimums and/or maximums observed in the years 1893 and 1984 correspondingly.

shown in Fig. 4(a) in this sense [Fig. 8(a)]. In other words, let us process the data with daily resolution; let time coordinates of temperature minimums and maximums (dmin- and dmax-coordinates correspondingly) be determined in accordance with Fig. 6(f); let misses be determined over and under $2 \sigma$-confidence interval [over- and under-misses in Figs. 8 (b) and 8 (c) correspondingly]. Then, changes 


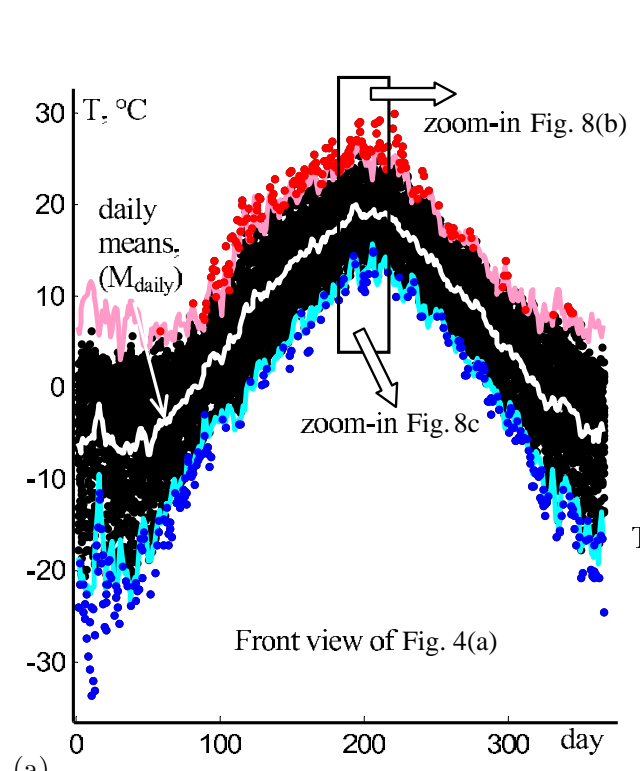

(a)

number of misses.

$\mathrm{T},{ }^{\circ} \mathrm{C} \quad \begin{aligned} & \text { (upper boundary } \\ & 30 \mathrm{daily}\end{aligned}$

averaged per year over 30-year window

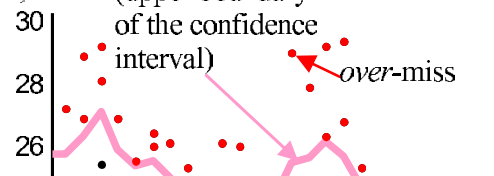

number of over-misses

per 30-years windows

24

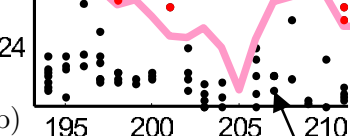

(b)

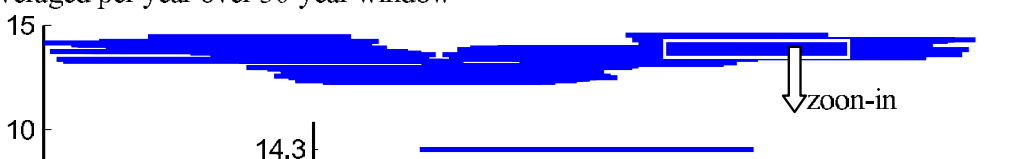

$\mathrm{M}_{\text {daily }}-2 \sigma_{\text {daily }}$

(lower boundary

of the confidence interval)
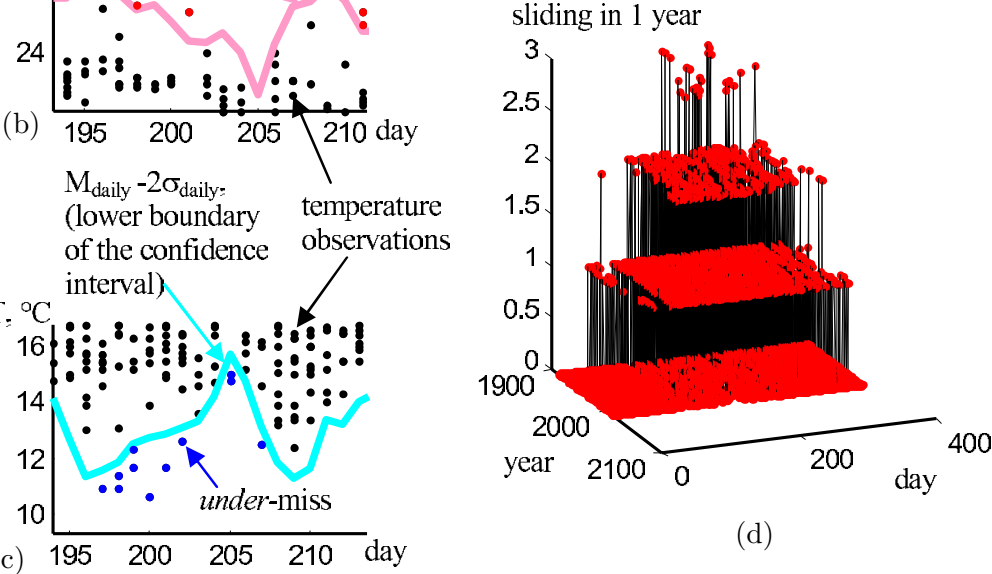

(d)

(c) $195200 \quad 205 \quad 210$ day

d)

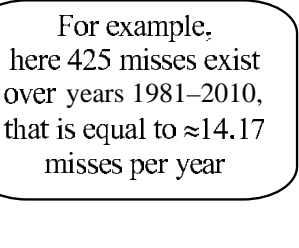

(e)

number of events

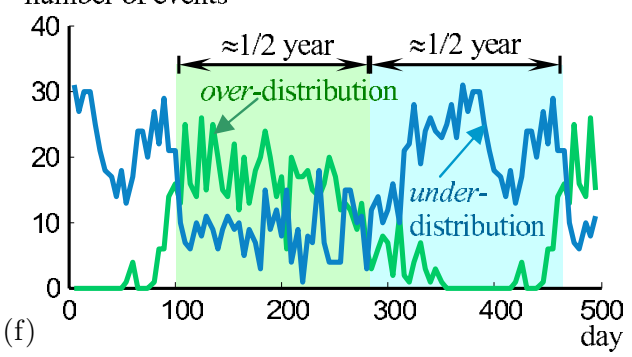

number

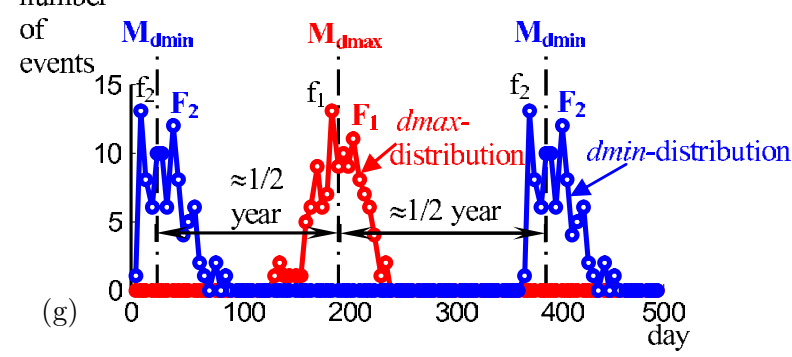

number of events

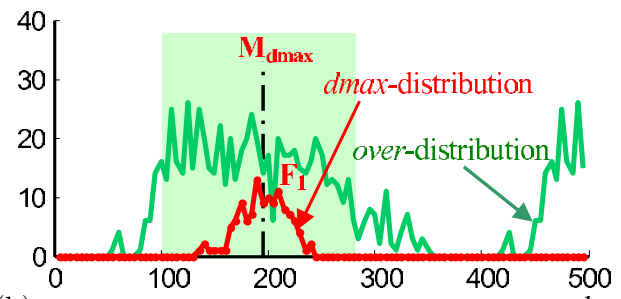

(h)

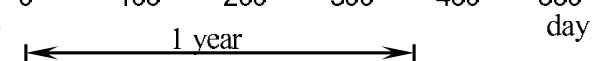

number of events

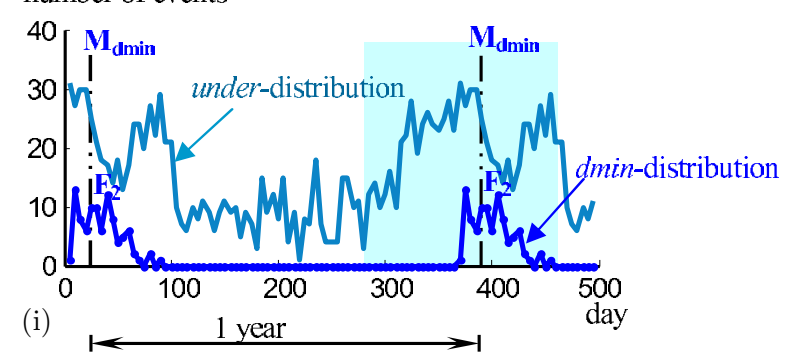

Fig. 8. Continued analysis of the example from Fig. 4(a): (a) over- and under-sets (red and blue points correspondingly) with relative confidence interval $\pm 2 \sigma$ (rose and azure broken lines) with (b) and (c) zoom-in views about the upper and lower boundaries of the confidence interval correspondingly; (d) distribution built for over-misses; (e) number of misses averaged per year within each 30-year window sliding in year; (f) over- and under-distributions; (g) dmin- and dmax-distributions; (h) and (i) combinations of over- with dmax-distributions and under- with dmin-distributions correspondingly. Figures (d)-(i) show the results of processing the temperature observations in St. Petersburg over years 1881-2015 [(d)-(e)] and over years $1891-2010[(\mathrm{f})-(\mathrm{i})]$. 
of local climate states estimated on the basis of these characteristics are practically absent throughout the meteorological observations, i.e. over the last 135 years at least. To illustrate it, let us show the following examples: dmin- and dmaxdependences over years 1881-2015 [for example, Fig. 6(g)]; distributions calculated for 30-year windows sliding in a calendar year over years 18812015 [for example, distributions of over-misses, Fig. 8(d)]; misses per year calculated for 30-year windows sliding in a calendar year over years $1881-$ 2015 [Fig. 8(e)]. Let us analyze the corresponding events over years $1891-2010$, i.e. over the term (120 years) which is divisible by $10,20,30,40$, 60 years. Let us build the following four distributions: for over- and under-misses [over- and underdistributions denoted by emerald and azure colors correspondingly in Fig. 8(f)]; for dmin- and dmaxcoordinates [dmin- and dmax-distributions denoted by blue and red colors correspondingly in Fig. 8(g)] And let us combine over-distribution with dmaxdistribution [Fig. 8(h)], and let us combine underdistributions with dmin-distributions [Fig. 8(i)]. In order to be more obvious, these results are grouped by sums in five days and are presented in the annual context.

Thus the over-distribution is in antiphase two times per year with the under-distribution [Fig. 8(f)]; each antiphase is about half a year long; the over-distribution dominates over a "warm halfyear" and the under-distribution dominates over a "cold half-year" (denoted by green and blue backgrounds correspondingly). Generally, dminand $d$ max-distributions demonstrate multimodality with the following centerline properties [Fig. 8 $8(\mathrm{~g})]$ : the mathematical expectations of these distributions $\left(M_{d \min }\right.$ and $\left.M_{d \max }\right)$ are in half-year from one another; there are two main modes about each expectation $\left(F_{1}\right.$ and $F_{2}$ are on the right, $f_{1}$ and $f_{2}$ are on the left). Next, the local slump of the over-distribution wave correlates with the dmaxdistribution centerline [Fig. 8(h)]; the local slump of the under-distribution wave correlates with the dmin-distribution centerline [Fig. 8(i)]. In this connection, the results of the statistical analysis based on the hypothesis on the normal distribution lead to the following conclusion: distributions of locations of annual temperature minimums and maximums show two significant modes at least (but not one mode which should exist under this hypothesis). In other words, deviations of time coordinates of temperature extremums in a daily scale cannot be explained by some noise-constituent only.

Various hypotheses on the physical nature of additional constituents can be proposed. Such hypotheses on climatic oscillations concern various periodical processes in paleo-climate and climate scales. for example Rial et al. . 2004: de Saedeleer et al., 2013: Cimatoribus et al. . 2014: Krauskopf \& Sieber, 2014; Oh et al., 2014; Williamson et al., 2016]. Of course, each of the physically-grounded constituents can influence the local climate dynamics more or less. However, the main question remains: which are the main constituents at present? Let us return to the temperature observations; and let us analyze these time series as signals. Let us continue to consider three cases: years 1891-2010 [similar to the extended statistical estimations over 120 years in Figs. 8(f)-8(i)] are analyzed in Figs. 9(a) and 9(b); years 1896-2015 [similar to the trend estimations by 40-year windows in Figs. 5(d)-5(h)] are analyzed in Figs. 9(c) and 9(d); years 1881-2015 [similar to the statistical estimations by 30 -year time windows sliding in a year over 135 years in Figs. 7(b), 7(c), 8(d) and 8(e)] are analyzed in Figs. 9(e) and 9(f). These results confirm the conclusion made from the extended statistical analysis, namely: 1-year harmonic dominates undoubtedly, because any of the remaining ones is less than $10 \%$ in relation to the module of Fouriertransformation for 1-year harmonic; a half-year harmonic is mainly the second one; other harmonics can appear from time to time (for example, 13.3years harmonic over years 1936-1975, 8-years harmonic over years 1976-2015, and so on).

The harmonic constituents (excluding 1-year harmonic) can theoretically mean various cases of periodic and quasi-periodic processes. In this connection, there is the periodical conception that originates from the conceptual model proposed by Lorenz 1963. Bifurcation diagrams built on the basis of Lorenz model simulations with variation of physically-grounded parameters show scenarios, where period doubling, intermittency, chaos and strange attractors can occur. Since 1-year harmonic identified by processing the temperature observations is too dominant (Fig. 9), then changes of this periodicity seem to be rare events for the local dynamics in a centennial term. Practically, use of the Lorenz model seems to be inherent for meteorological needs due to the so-called butterfly-effect which is discussed in the wide sense by Palmer et al., 2014]. 


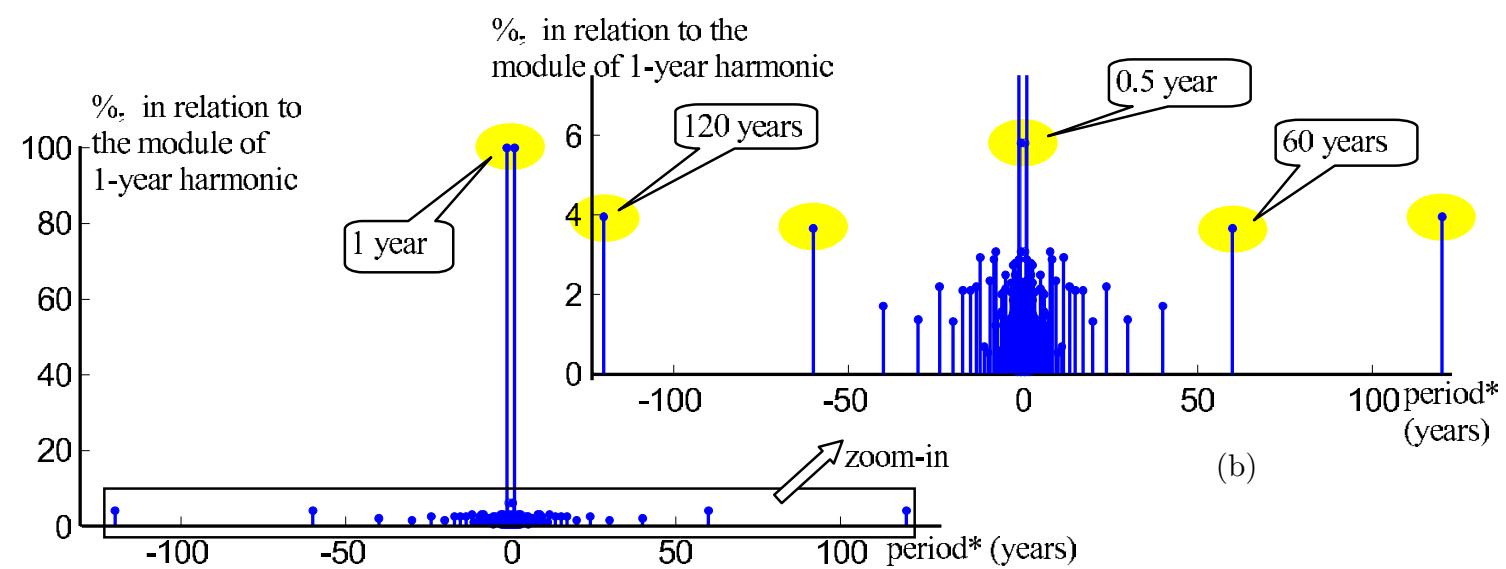

(a)
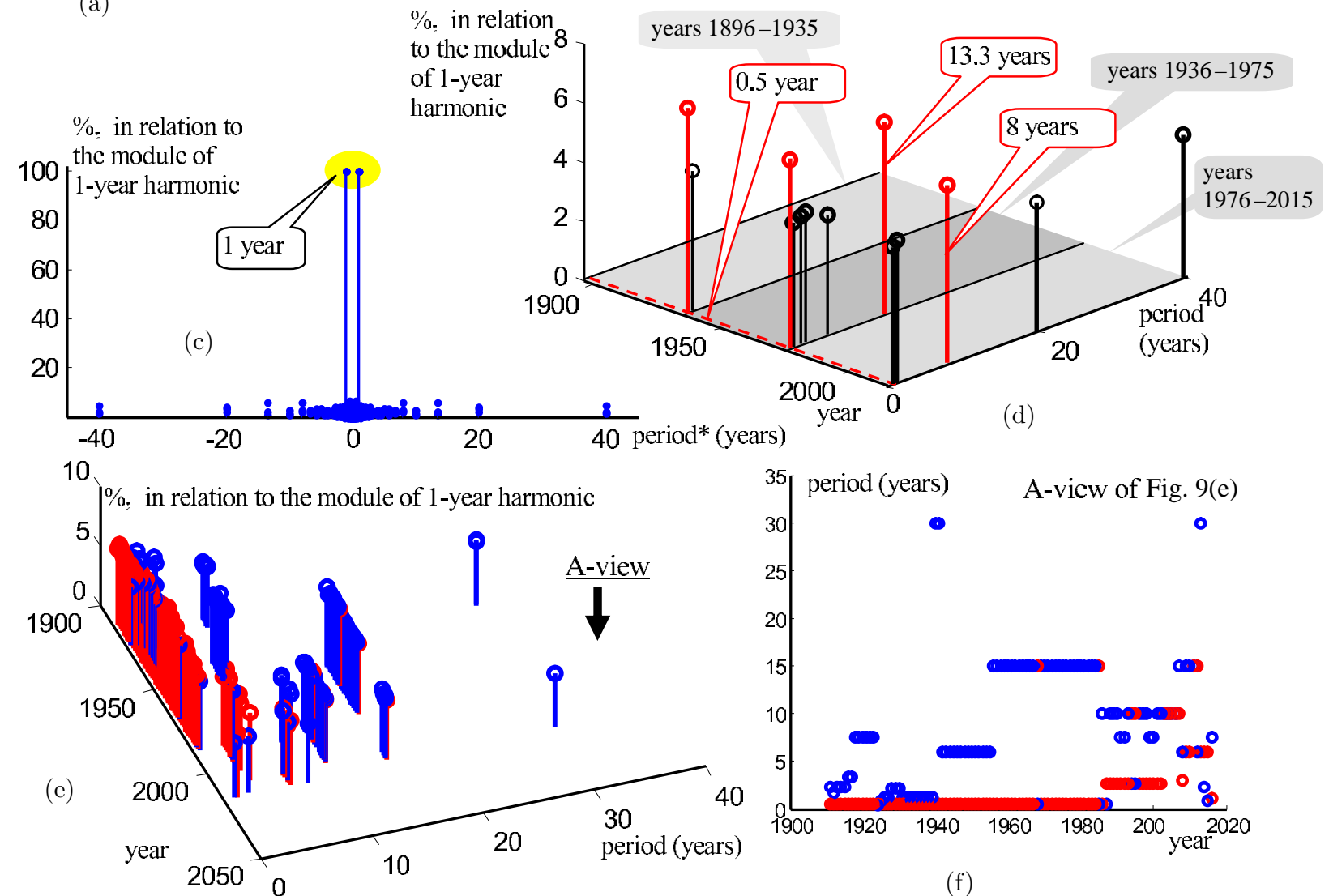

(f)

Fig. 9. Results of Fourier analysis, where the abscissa axis shows the relative module of Fourier-transformation and the ordinate axis shows periods recalculated from the corresponding harmonics (for obviousness): (a) and (b) a general view and zoom-in fragment identified over years 1891-2010 (similar to the estimations over 120 years discussed in Figs. 8)(f)-8)(i); (c) and (d) general view and details on the significant harmonics (5-10\%) identified over years 1896-2015 (similar to the trend estimations discussed in Figs. 5(d) 5 h); (e) and (f) two views to illustrate details on significant harmonics (5-10\%) identified by 30-year windows sliding in a year over years 1881-2015 [similar to Figs. [7 b), [7] c), 8(d) and 8] e)]. The second harmonics are denoted by red bars in (d)-(f) under the fact that 1-year harmonic is the first one (and is equal to $100 \%$ ).

Thus terms of several days long are taken into considerations, for example Trevisan \& Palatella, 2011; Uboldi \& Trevisan, 2015; Sooraksa \& Chen, 2018]. High-order Lorenz-like models demonstrate quasiperiodic processes, including cases with two central peaks about maximum and minimum, for example Faghih-Naini \& Shen, 2018]. However, quasi-periodic solutions are inadmissible for the original Lorenz model dynamics in accordance with the restrictions on its consistency from the physical validation Berge et al., 1988. Attempts to justify the observed abnormalities by bifurcations 


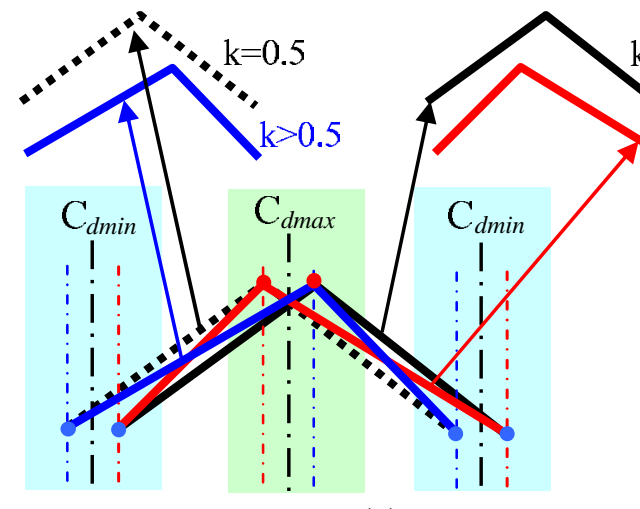

(a)

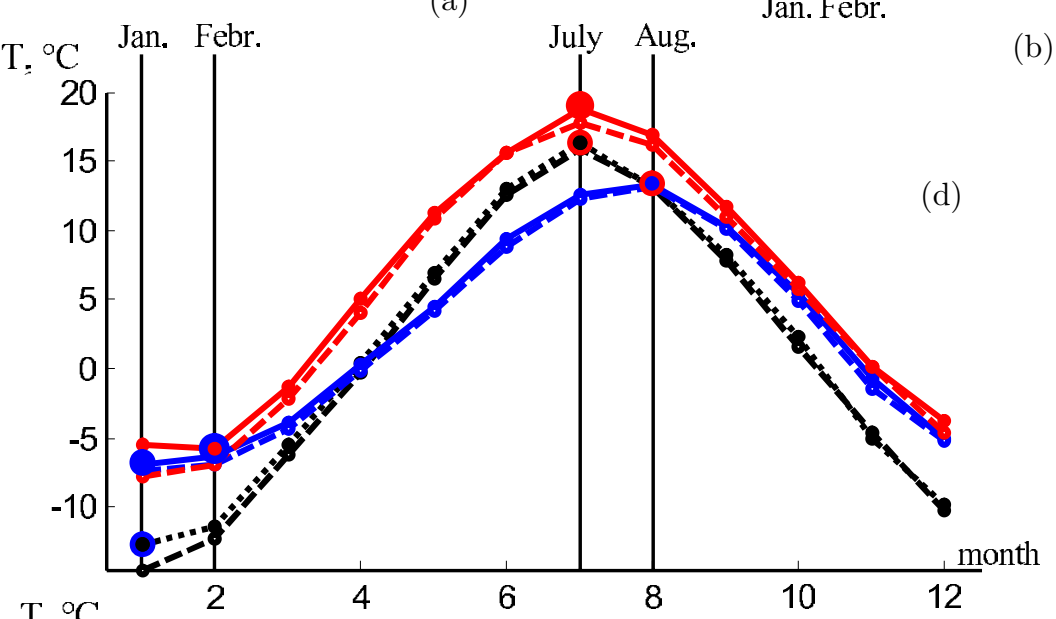

b) $k$ is a relative duration of warming stage over year

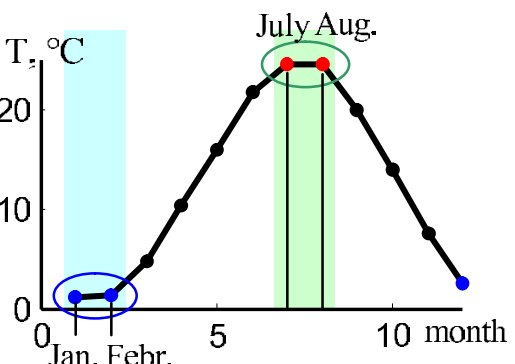

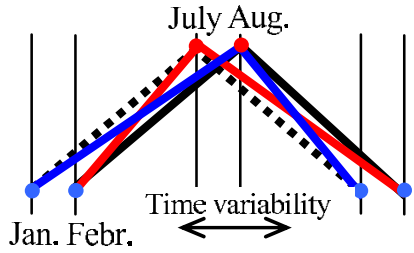

(c)

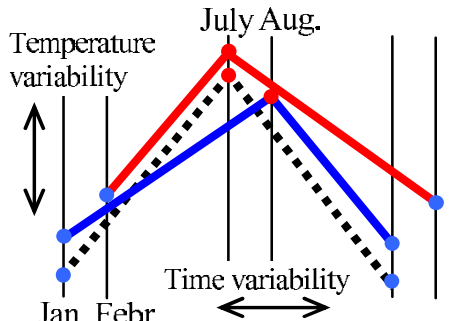

(e)

Climate norms built for:

St. Petersburg over years 1961-1990 - over years 1981-2010

Arkhangelsk over years 1961-1990 - over years 1981-2010 ...... Petropavlovsk-Kamchatskyi over years 1961-1990 - -

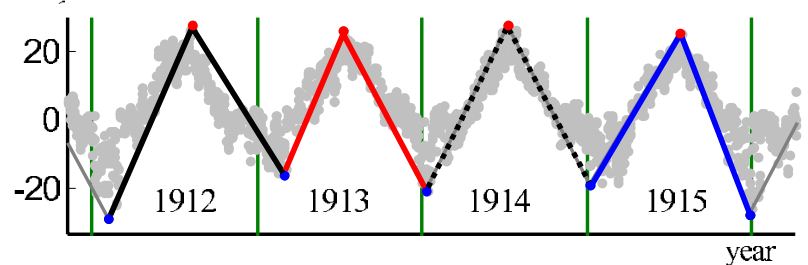

(f)
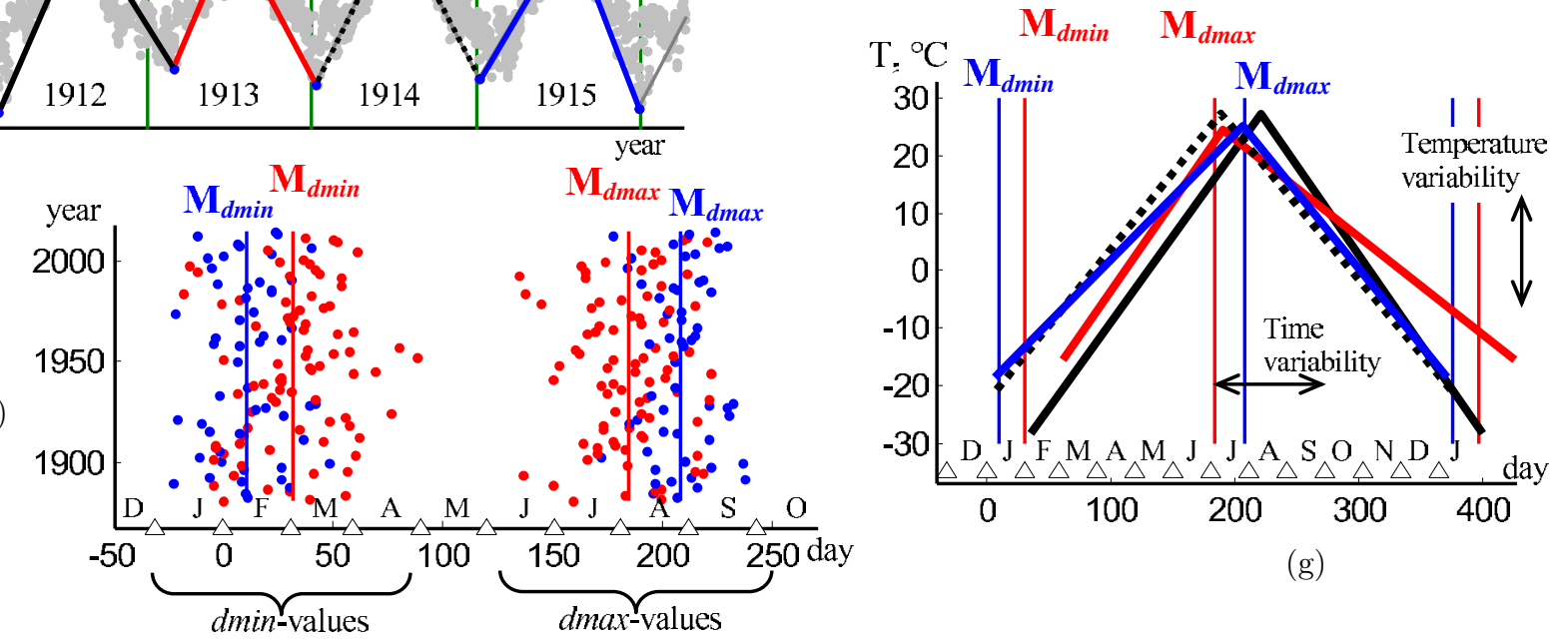

Fig. 10. (a) A scheme to the ensemble of four elemental processes; (b) and (c) the climate norm built for Mahachkala (WMOcode 37472) over years 1981-2010 and a scheme to effects of time variability correspondingly; (d) and (e) combination of the climate norms built for St. Petersburg (WMO-code 26063), Arkhangelsk (WMO-code 22550), Petropavlovsk-Kamchatskyi (WMO-code 32583) to show three cases of the elemental processes in monthly terms and a scheme to time-and-temperature variability correspondingly; (f) and (g) an example of four successive annual temperature cycles to show four elemental processes in daily terms and their time-and-temperature variability correspondingly; (h) mathematical expectations of annual maximums (red points) and annual minimums (blue points) combined in (day, year)-space. Results presented in (f)-(h) are for St. Petersburg. 
simulated via Lorenz-like models, for example Bartsev et al., 2017], show too large changes of both amplitude and relief of time series which have not been observed over the last 135 years at least. So, other ways should be found to apply the Lorenz model in order to justify the interannual variability under the assumption that this model is pertinent to such time scale.

The regulator conception originates from the conceptual model of the hysteresis regulator with double synchronization (so-called $H D S$-model) as proposed in Kolokolov \& Monovskaya, 2015a, $2015 \mathrm{~b}$. In this case, the effects of the interannual variability are treated as trivial events from alternations between several elementary processes with the same periodicity and different structures of phase trajectories. In terms of the sketch in Fig. 6)(a), this means 1-year periodicity and four combinations of extremums [Fig. 10(a)]: $k>0.5$ (denoted by blue), $k<0.5$ (denoted by red), and two cases with $k=0.5$ (denoted by solid and dotted black lines), where $k$ is a warming stage within one year. Then a climate norm supposes one of these combinations for each time window [Fig. 4(e)] in spite of the fact that swings of monthly extremes are essentially more various [Fig. 7 (c)]. Extremums of two months long for one local climate system appear only sometimes [for example, Fig. [10(b)] and show the effect of time variability [Fig. 10(c)]. Climate norms built for several local systems [for example, Fig. 10(d)] can show time-and-temperature variability [Fig. 10(e)]. Daily resolution confirms the ability of alternating four combinations for each local climate system [for example, Fig. [10)(f)] with effects of time-and-temperature variabilty [Fig. 10(g)]. Generally, the positional relationship for cases with $k>0.5$ and $k<0.5$ [denoted by blue and red points in Fig. 10(h) correspondingly] confirms the scheme in Fig.6(a): the average minimum for the cases with $k>0.5$ (blue $M_{d \min }$ ) is on the left from the average minimum for the cases with $k<0.5$ (red $\left.M_{d m i n}\right)$; the average maximum for the cases with $k>0.5$ (blue $M_{d \max }$ ) is on the right from the average maximum for the cases with $k<0.5\left(\right.$ red $\left.M_{d \max }\right)$. To discuss local climate dynamics from the regulator conception, let us focus briefly on the $H D S$-model.

\section{HDS-Law and Peculiarities of Modified Bifurcation Diagrams}

The $H D S$-regulator was first proposed in [Zaytsev et al., 1975] and refined to the present variant in
Kolokolov et al., 1991]; results of the bifurcation analysis on $H D S$-regulator dynamics were first presented in Kolokolov et al., 2003]; the HDS-model was first proposed to describe the dynamics of local climate systems in Kolokolov \& Monovskava, 2015a]. Let us summarize the aspects which seem to be important to comprehend the observed temperature phenomena in the dynamics of local climate systems. To provide a clear explanation, annual warming-cooling cycles are mainly described by a linearized view [Fig. 11(a)]. On the one hand, such view resembles more closely the form of solutions on interannual forecasting from the traditional viewpoint (i.e. seasonal annual temperature variation, Fig. 2). On the other hand, it is enough to consider conceptually the basic regularities and evolutional mechanisms from the regulator viewpoint. Certainly, it simplifies the observed nonlinear waveforms; however, annual waveforms can seem close to the linearized view. For example, 1889-year cycle in Fig. 6(b), 1984-year cycle in Fig. 7(e), and 1912year cycle in Fig. 10(f) could be attributed rather to nonlinear waveforms; 1890-year cycle in Fig. 6(b), 1893-year cycle in Fig. 7(d), and 1914-year cycle in Fig. 10(f) could be attributed rather to linear waveforms. Thus, it seems to be possible to consider the linear view as a particular case of the nonlinear view. And there are results, for example Kolokolov \& Monovskava, 2016b, 2016c], which justify the applicability of the $H D S$-model for both linear and nonlinear views.

The HDS-law is described by the following control algorithm formalized by the commutation function $\left(K_{F}\right)$ :

$$
K_{F}(t)= \begin{cases}1, & \text { if } x(t)>\frac{H}{2}, \\ 0, & \text { if } x(t)<-\frac{H}{2}, \\ 0 \rightarrow 1, & \text { if } t=j T_{S} \text { or } x(t)=\frac{H}{2}, \\ 1 \rightarrow 0, & \text { if } t=(j+0.5) T_{S} \text { or } \\ & x(t)=-\frac{H}{2},\end{cases}
$$

where $x$ is a phase variable to establish the correlation between a local daily mean temperature $(T)$ and regulative restrictions on a local temperature reference $\left(T_{\mathrm{ref}}\right), x=T_{\mathrm{ref}}-\beta T ; \beta$ is an amplification 


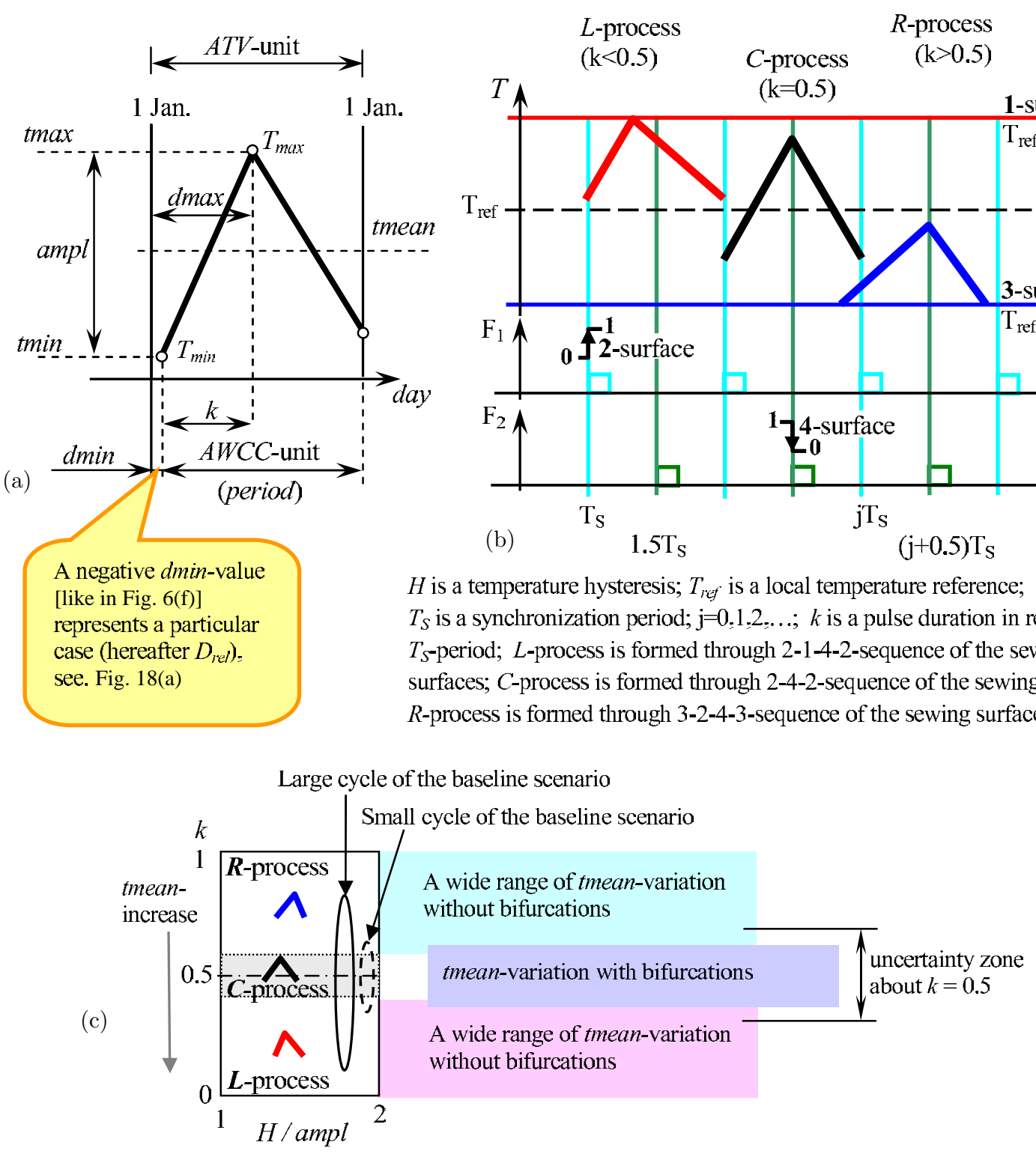

Fig. 11. (a) Diagram to illustrate the $A W C C$-unit characteristics; (b) diagram to illustrate how the elementary processes of the $H D S$-model are realized in accordance with the commutation function to determine the moments of the structural changes and (c) a simplified sketch to the parametrical diagram in $(H / a m p l, k)$-space to explain the peculiarities of the baseline scenario of $H D S$-dynamics evolution.

coefficient and hereafter $\beta=1 ; H$ is a local temperature hysteresis to restrict amplitude pulsations about an average; $T_{S}$ is a synchronization period (1 year); $j=0,1,2, \ldots$ is a number of the running period. The simplest view of the dynamical system is the following:

$$
\frac{d x}{d t}=a x+b\left(K_{F}\right)+c,
$$

where $a, b, c$ are parameters.

So, $K_{F}$-function determines the moments of structural changes of a phase trajectory in accordance with four surfaces [Fig. 11(b)]: two surfaces (denoted by "1" and "3") which are sew phase trajectories by level; two surfaces (denoted by "2" and "4") sew phase trajectories by time. Each of the moments of structural changes is a result of the natural competition between the time quantization (the double synchronization) and the amplitude quantization (the temperature hysteresis). Two synchronization sequences $\left[F_{1^{-}}\right.$and $F_{2}$-sequences in Fig. [11(b)] of equal frequency $\left(1 / T_{S}\right)$ with $T_{S} / 2$-shift in time restrict durations of warming and cooling stages. In the physical sense, 
such time synchronization seems to be concordant with maximal and minimal distances between the Earth and the Sun (aphelion and perihelion correspondingly); a temperature hysteresis seems to be concordant with a local Earth surface heat budget as used in Budvko, 1967; Stephens et al., 2012; von Schuckmann et al., 2012]. So, in accordance with the $H D S$-hypothesis, local climate dynamics is described conceptually by a dynamical model (i.e. by the $H D S$-model) under the algorithm described by Eq. (1).

From the control theory viewpoint, the timeand-amplitude competition provides the heightened stability of energy conversion processes with $T_{S^{-}}$ periodicity at the expense of specific regular modifications of phase patterns. From the nonlinear dynamics viewpoint, these modifications are mainly described by three elementary processes with the same periodicity $\left(T_{S}\right)$ and different orders of the structural changes [Fig. 11(b)]: $R$-process (rightprocess through 3-2-4-3 surfaces) with $k>0.5$; $C$-process (central-process through 2-4-2-surfaces) with $k=0.5 ; L$-process (left-process through 2 1-4-2 surfaces) with $k<0.5$, where $k$ is the main bifurcation parameter. Researches of nonlinear dynamics of $H D S$-regulators, for example Kolokolov et al., 2003; Kolokolov \& Monovskava, 2015e, 2015f], show that $(H / a m p l, k)$-space is the most illustrative to the base of $H D S$-dynamics evolution. Namely [Fig. [11(c)], $R-, C$-, $L$-processes occupy practically fully $(H / a m p l, k)$-space on the right of $H / a m p l=1$ and are symmetrically disposed in that space in relation to $k=0.5$. So, from the regulator conception, the interannual variability is justified by alternations between the elementary periodical processes (i.e. different variants of 1year periodical processes) instead of chaotic fluctuations about average annual extremes in accordance with the probable conception and instead of changes of periodicity in accordance with the periodical conception.

A transition from one elementary periodical process to another is realized through so-called Cbifurcation (or in other words, through a bordercollision bifurcation); such alternating transitions look like so-called intermittency phenomenon. Generally, various border-collision bifurcations are typical for dynamical systems with variable structures, for example Feigin. 1978: Tse. 2004: El Aroudi et al.,2005]. Intermittency phenomena are identified in the dynamics of nonlinear systems of different nature, including the intermittency between periodic and strange attractors in the dynamics of the Lorenz system Berge et al., 1988]. However, for the majority of the known nonlinear systems, intermittency phenomena are either particular atypical cases or even extremely undesirable cases. In this sense, such phenomena are rarely considered and relate either to purely scientific (i.e. far from practice) considerations on nonlinear potentialities connected with intermittency effects or to isolated instances of specific practice-oriented investigations aimed at the prevention of intermittency effects. Moreover, intermittency phenomena represent extremely "capricious" nonlinear behaviors, for example Kolokolov \& Monovskaya, 2013a], because the abilities of classical bifurcation diagrams are originally insufficient for a clear analysis and there are essential differences between model-based and experimental realizations of such phenomena.

In the context of the HDS-hypothesis, it is supposed that intermittency-like behaviors form some permanent regime and it is necessary to answer the question on regularities and tendencies inherent to this regime. Classifications of intermittency can originate from different types of bifurcations, for example Berge et al., 1988. However, how do we classify intermittency of the same type (border-collision bifurcations between three elementary processes with the same periodicity)? Next, alternations between periodical processes are usually mapped by classic bifurcation diagrams as a process, periodicity of which corresponds to no case among the alternating processes. For example, alternations between 1-process and 2-process about the bifurcation boundary show mainly 3process or divisible-by-3-process [circled by orange in Fig. 12(a)]. In order to avoid such distortion of real dynamics, it is necessary to make some modifications of the diagrams. Such modifications should be enriched by additional information (for example, stability shades, transients, intermittency, operating performance, and so on); as a result, these modifications become inevitably dependent on purposes, for example Kolokolov \& Monovskava, 2006, 2013a, 2013b, 2015a, 2015b, 2015c, 2016a, 2016b, 2016c, 2018]. Thus, it becomes a point of practice-oriented modified bifurcation diagrams specialized for analytics based on experimental time series (namely, temperature observations). 

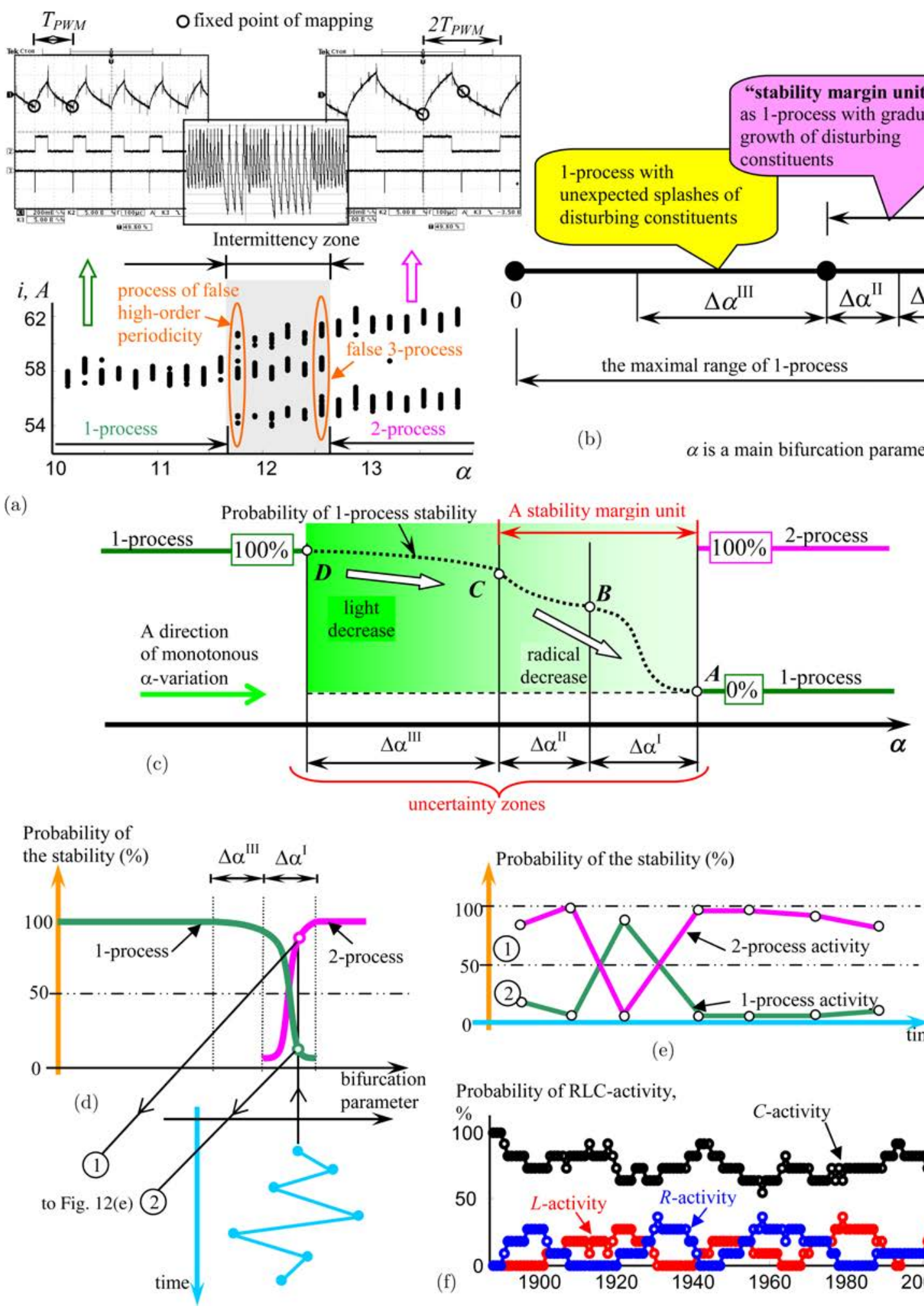

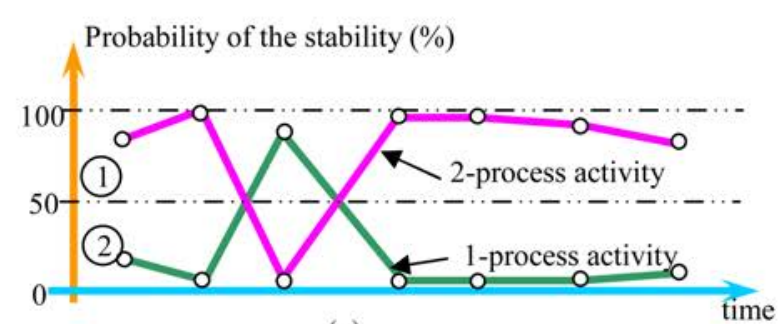

(e)

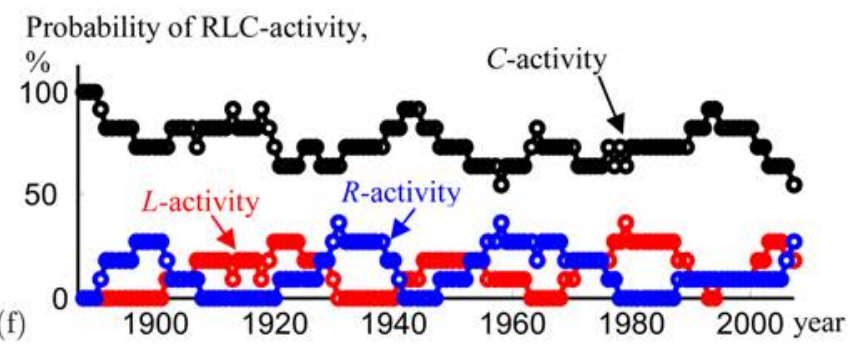

Fig. 12. Consideration on 1-2-4-... scenario adapted from Kolokolov \& Monovskaya, 2013a, 2013b]: (a) a bifurcation diagram made by processing experimental data from a PWM-converter with the range of intermittency phenomena; (b) and (c) schemes to illustrate the structure of 1-process uncertainty zone without and with probable characteristics, correspondingly, with monotonous variation of a bifurcation parameter. Here PWM means a pulse-width modulation control. (d) and (e) Interrelations between 1- and 2-processes in the probable sense with monotonous and probable parametrical variations correspondingly. (f) The modified bifurcation diagram built for Nijnyi Novgorod (WMO-code 27459) to show the evolution of the elementary processes over time in the probable sense. 
Modified bifurcation diagrams oriented to processing the temperature observations was first proposed in Kolokolov \& Monovskaya, 2015a. Briefly, it was the idea on how to decompose complex behaviors within a bifurcation zone into constituents and how to visualize their interrelations over time. In this connection, let us take into account the regularities of stability degradation shown by the method of uncertainty zones for technical systems with variable structures, for example Kolokolov \& Monovskaya, 2013a], where analytics is mainly focused on the first and second dynamical processes within 1-24-... scenario [Fig. [12(a)]. Then intricate interrelations between nonlinear and noise constituents towards a bifurcation point are decomposed into uncertainty zones of three kinds [Fig. 12(b)], where disturbing constituents can either grow gradually or appear unexpectedly. In this context, the radical decrease in stability of a dynamical process is observed within a so-called stability margin unit consisting of the uncertainty zones of the first and second kinds $\left[\Delta \alpha^{I}\right.$-zone and $\Delta \alpha^{I I}$-zone correspondingly, Fig. [12(c)]. However, the majority of the experimentally revealed intermittency phenomena are realized within $\Delta \alpha^{I}$-zone which is formed under the dominating hysteresis effects caused by parametrical drifts over time. At the same time, in the cases of border-collision bifurcations, $\Delta \alpha^{I I}$-zone range is essentially lesser than $\Delta \alpha^{I}$-zone range which allows to neglect $\Delta \alpha^{I I}$-zone [Fig. 12(d) and hereafter].

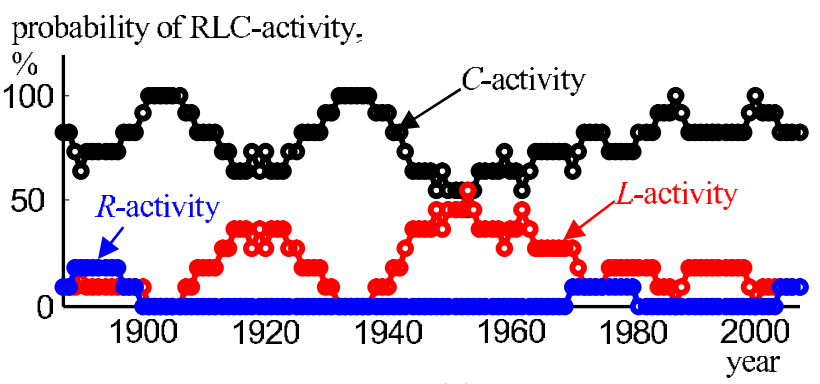

(a)

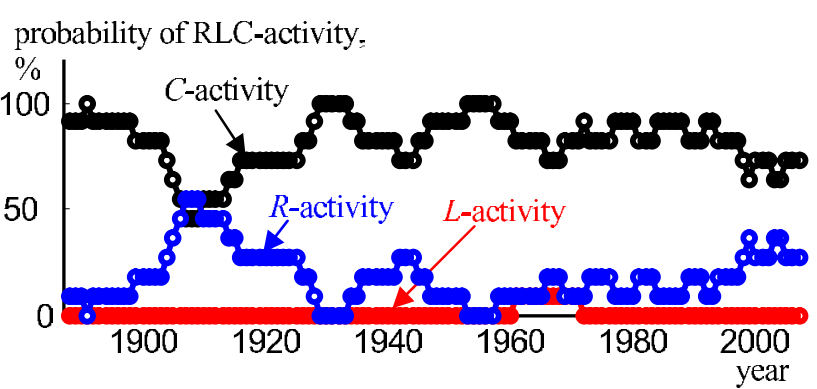

(b)

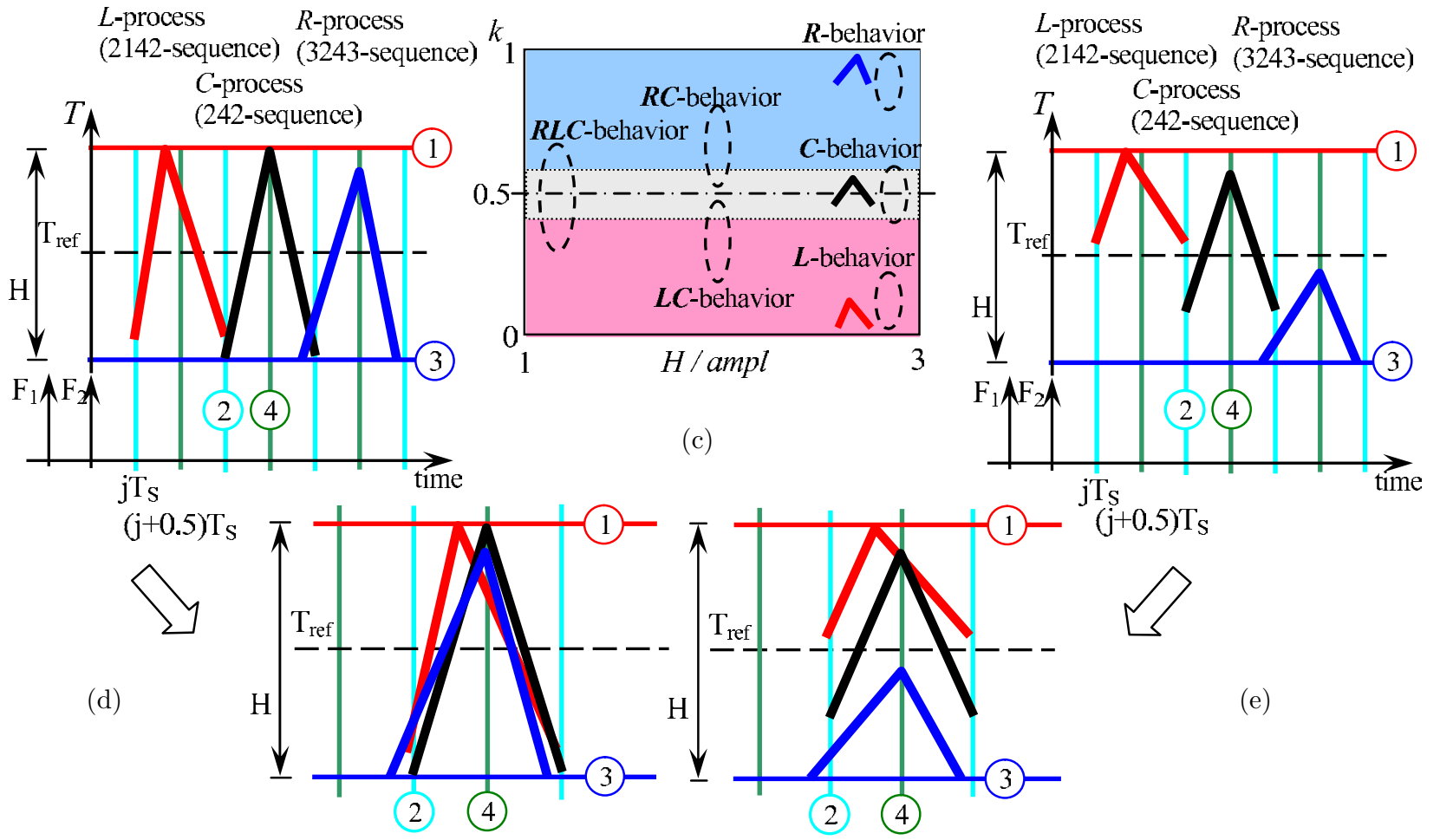

Fig. 13. (a) and (b) The modified bifurcation diagrams built for St. Petersburg (WMO-code 26063) and AlexandrovSahalinskyi (WMO-code 32061) to illustrate interrelated activities of the elementary processes for $L C$-behavior and $R C$ behavior correspondingly; (c) locations of six kinds of behaviors in $(H / a m p l, k)$-space; (d) and (e) diagrams illustrating phase peculiarities of the elementary processes close to $H / a m p l=1$ and far from $H / a m p l=1$ correspondingly. 
So, let us focus on two neighboring dynamical processes within the scenario [1- and 2-processes, dominating probabilities of which are denoted in Fig. 12(d) by green and magenta curves correspondingly]. These processes change their probabilities intensively with monotonous variation of a bifurcation parameter. Let combinations of these probabilities with each bifurcation parameter [for example, points 1 and 2 in Fig. 12(d)] remain true for nonmonotonous parametrical variation [for example, blue broken line in Fig. [12(d)]. Then each parametrical value can be associated with the corresponding pair of probabilities, and a sequence of such combinations over time forms synchronized time series to show interrelated activities of these processes [for example, 1-process activity and 2-process activity denoted by green and magenta broken lines in Fig. 12(e)]. So, a complex behavior (like intermittency phenomenon) can be associated with the evolution of constituents presented in such probable sense; and the number of processes involved in such behavior can be more than two.

This idea was applied for analytics of the temperature observations. for example [Kolokolov \& Monovskaya, 2015a, 2015b, 2015c, 2016a, 2016b, 2016c, 2017a, 2017b, 2017c] from the assumption that the HDS-hypothesis is conceptually true. Then each temperature time series was transformed into an ensemble consisting of three constituents (i.e. constituents of $R$-, $L-, C$-processes), interrelated evolution of which over time is described by the corresponding activities for example, Fig. 12(f). These results show that the baseline evolutional scenario observed in the local climate dynamics over the last 135 years can be reduced to intermittency-like behaviors between $R$-, $L$-, $C$-processes [for example, Fig. 11(c)]. Both order and repetition of the constituents are usually unique for each local climate system; however, the observed kinds of patterns are mainly restricted by three cases: $L$-activity dominates over $R$-activity [ $L C$-behavior, Fig. $13(\mathrm{a})] ; R$ activity dominates over $L$-activity $[R C$-behavior, Fig. 13(b)]; $L$ - and $R$-activities are comparable [RLC-behavior, Fig. 12(f)]. Differences between the parametrical and phase volumes suppose that significant temperature changes can be realized without bifurcations [Fig. 11(c), blue and rose ranges]; and, vice versa, subtle temperature changes can be realized on the background of quite intensive intermittency [Fig. 11(c), violet range]. Proximity of $R-, L-, C$-processes in the phase space depends on
$\mathrm{H} / \mathrm{ampl}$-value [Fig. 13)(c)]: the larger the $\mathrm{H} / \mathrm{ampl}$ value is, the farther $R$ - and $L$-processes are from each other [Fig.[13(e) in comparison with Fig. [13(d)]. So, essential shifts of annual extremums in time can be realized with/without significant temperature changes. Let us develop the $H D S$-hypothesis for truth (Sec. 55) and perspective (Sec. 6).

\section{Arguments for the HDS-Hypothesis}

We believe that progress in research on evolution of the local climate dynamics is hindered by the necessity to satisfy two interrelated demands: oddities observed in the dynamics should be described by a conceptual model in the form of a dynamical system; analytical tools should take into account local time and temperature details concerning daily means at least. Why do we connect these demands? Because, on the one hand, if such conceptual model is known then expected behaviors can be modeled; so, the specialized tools to analyze the observed dynamics (as expected behaviors) could be chosen and a valid answer on the bifurcation evolution could be done. On the other hand, if universal tools to analyze all the nonlinear phenomena could be known then all the behaviors of the observed dynamics could be distinguished; so, a conceptual model to collect these behaviors could be chosen and a valid answer on the bifurcation evolution could be done. However, bifurcation diagrams built by processing the local temperature observations over the last century were absent before the $H D S$ hypothesis was proposed. Perhaps, that looks like an extraordinary combination: the pulse regulator which is not widely used; the ensemble of elementary processes in contrast to an annual behavior; intermittency-like phenomena as the basic behavioral scenario in contrast to the habitual periodic processes. However, this is not contrary to nonlinear dynamics conceptions and is logically consistent.

So, let the $H D S$-hypothesis be conceptually true. Then this means the necessity to analyze in detail the intermittency on the background of parametrical drifts, where various transient effects seem to be inevitable. The traditional analytical tools (the statistical analysis, time-and-frequency transformations, and classical bifurcation analysis) are mainly intended to consider stationary states and, correspondingly, cannot directly be used for such transient effects. For example, let us formally 

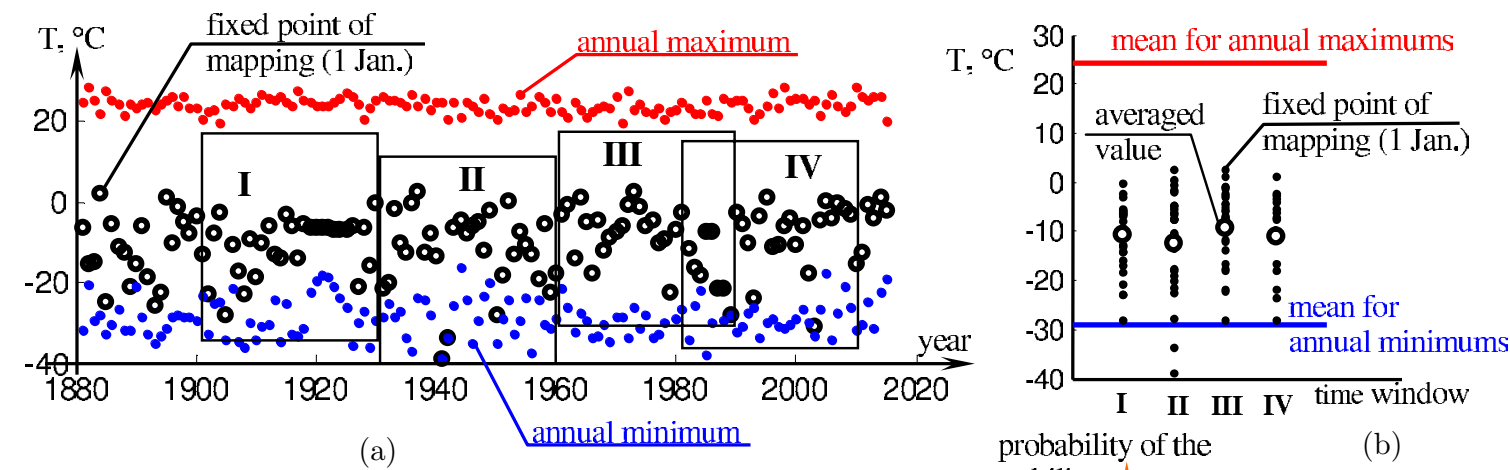

probability of the

(b)

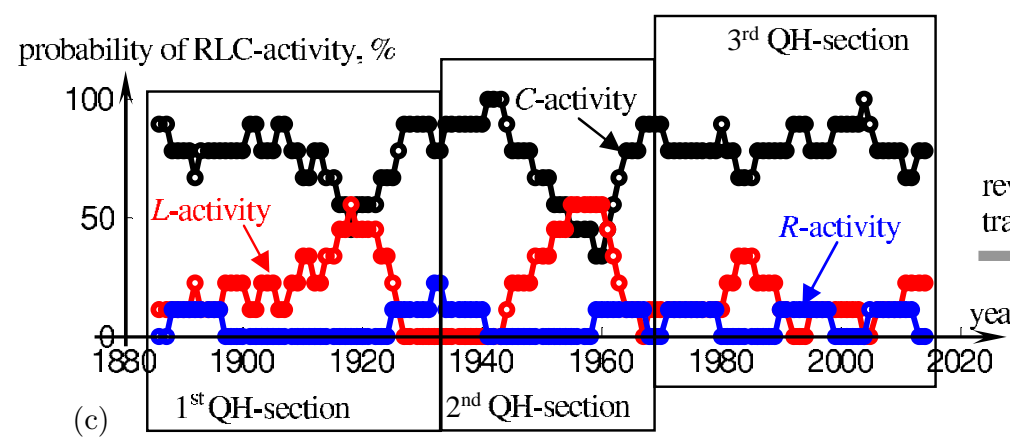

stability,
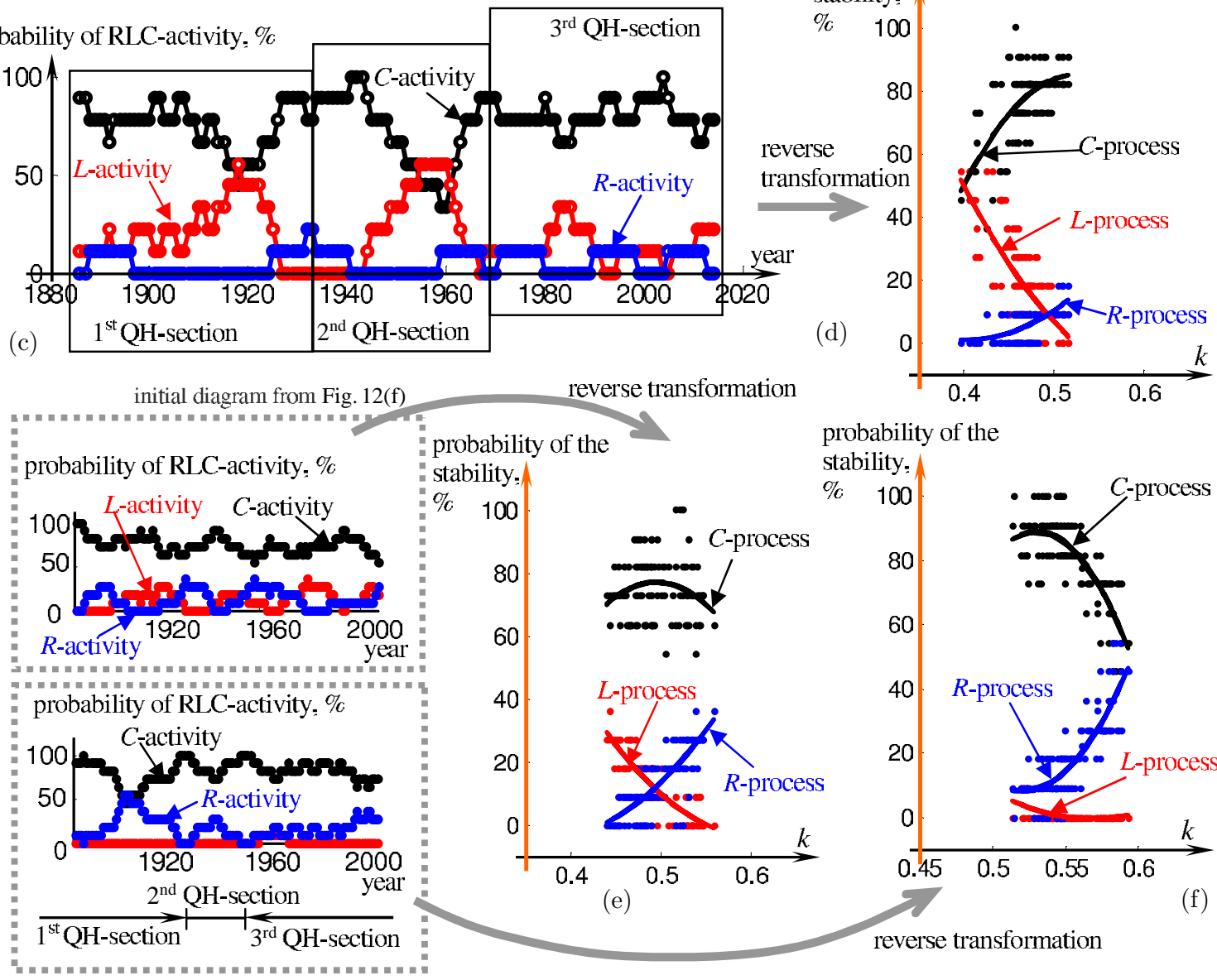

reverse transformation

probability of the

probability of the stability: stability.

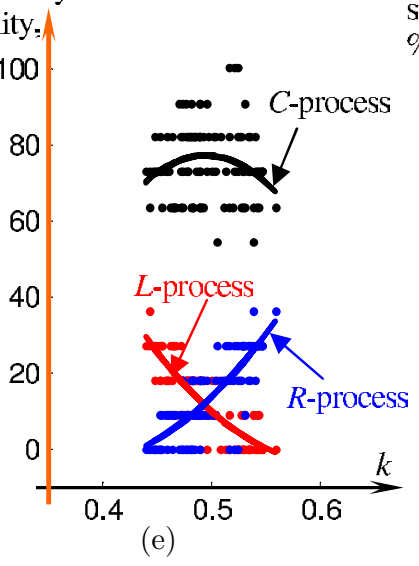
$\% \quad 100$

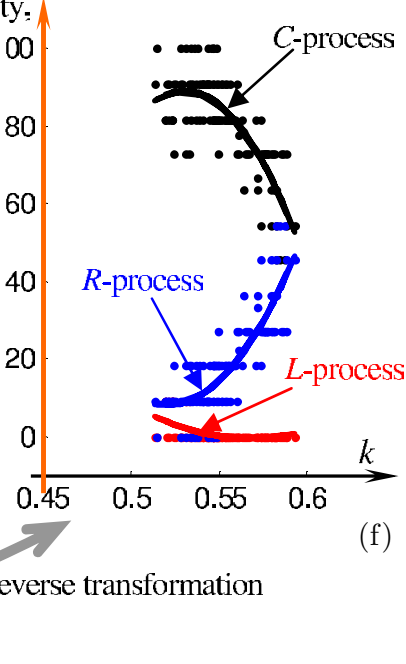

Fig. 14. Results of processing the temperature observations made in Vutegra (WMO-code 22837): (a) fixed points of mapping on January 1 (white points circled by black) combined with annual temperature maximums and minimums (red and blue points correspondingly); (b) the bifurcation diagram built by four samples of the fixed points of mapping (black points) averaged over 30 years (four white points circled by black) combined with the averaged levels of the annual maximums and minimums (red and blue lines correspondingly); (c) and (d) the modified bifurcation diagram to show evolution of $R L C$-activities over time and the reverse transformation of this diagram to the probable view with $k$-variation. (e) and (f) Results of the reverse transformation of the modified bifurcation diagrams to the probable view with $k$-variation built for Nijnyi Novgorod and Alexandrov-Sahalinskyi correspondingly.

build a classical bifurcation diagram to show phase variation over time in (time, temperature)-space [Fig. 14(a)]. From the uni-behavior conception, the fixed points of mapping should be grouped by 30years [I, II, III, and IV time windows, Fig. 14(a)] and the corresponding bifurcation sections look like a chaotic solution or a quasi-periodic solution [Fig. [14(b)]. The modified bifurcation diagram built for the same temperature observations [described in Sec. 4 by Figs. 12(a)-12(f)] is shown in Fig. 14(c). 
Such diagram is specialized to clarify evolutionary tendencies expected in the context of the intermittency in accordance with the $H D S$-model, namely: generally, there are three constituents $(R-, L-, C$ activities); in particular, $L C$-behavior occurs and three evolutionary cycles are realized. Per se, it is assumed that a modified bifurcation diagram on $R L C$-activities [for example, Fig. 14(c)] represents a particular realization of the dynamics evolution over time according to the general probabilistic interrelations between $R-, L-, C$-processes within the bifurcation scenario [for example, Fig. 14(d)]. Let us verify this assumption by the rule of contraries.

Then it should be possible to make the reverse transformation from the $R L C$-activities to the interrelated probabilities in the general context of the stability degradation according to the baseline evolutionary scenario, where the main bifurcation parameter $(k)$ is strictly prescribed. The result of this transformation is presented in Fig. 14(d). And let us accompany this case by reverse transformations built in similar manner for the remaining cases: for $R L C$-activity [Fig. 14(e)] and for $R C$-activity [Fig. 14(f)]. Let us explain why these results seem to be successful. With this purpose, let us return to Fig. 12(d), where a typical probable structure of a bifurcation range connecting two neighboring periodical processes is shown. So, let us repeat this consideration for the bifurcation range connecting the next pair of the neighboring periodical processes [Fig. [15(a), upper part]. Next, let us suppose that $k$ is the bifurcation parameter, and let us concord $L-, C$-, $R$-processes with this structure [Fig. 15)(a), lower part]. Then, $C$-process range should be reduced practically to zero due to the $H D S$-dynamics peculiarities in the parametrical space [Fig. 11(c)]. In other words, this means that points A and B in Fig. 15(a) should be united, and two bifurcation ranges are transformed into the structure presented in Fig. 15(b). Then idealized views of the modified bifurcation diagrams for $R C$-, $R L C$-, and $L C$-behaviors with $k$-variation about $k=0.5$ should look like the ones presented in Figs.15(c)-15(e)(upper parts). And these diagrams are concordant with the results from processing the temperature observations [i.e. Figs. 15(c)-15(e) are concordant with Figs. 14(d)-14(f) correspondingly], and that proves the assumption accepted to build the modified bifurcation diagrams to show the evolution of the elementary processes over time.
Each modified bifurcation diagram of $R L C$ evolution reveals the unique pattern of time windows, during each of which the local climate dynamics passes through an evolutionary cycle by analogy with an annual cycle of temperature evolution or with an 11-year cycle of solar evolution. In this sense, each cycle of $R L C$-evolution demonstrates quasi-homogeneous dynamics in a climate scale, as denoted by a $Q H$-section in KKolokolov \& Monovskaya, 2016b, 2016c]. So, a $Q H$-section supposes two-way $k$-variation, with which one of the dominant tendencies ( $L$-, $R$ - or $C$-activity) determines evolutionary changes. For example, the modified bifurcation diagram shown in Fig. 14(c) demonstrates three $Q H$-sections determined by $L$-activity. Summary of $Q H$-sections determined for climate systems located in European and Asian parts of Russia is presented in Fig. 15(f). Here, the cycles of 30-years long dominate among other cycles, so, perhaps, the choice of 30-year windows for the conventional methods of climate dynamics analytics had reasons. Also, perhaps, these results can confirm the influence of Pacific Decadal Oscillations and North Atlantic coupled ocean-atmosphere variability on the regional climate. Nevertheless, the $H D S$-hypothesis persists in the following: patterns of $Q H$-windows for local climate systems are characterized by variable durations and beginning dates in contrast to the unified 30-year windows from unibehavior conception. The reason for the heterogeneity of $Q H$-windows is connected with so-called likely periodicity formulated in Kolokolov \& Monovskaya, 2015b]: each sunspot extremum provides the possibility to change qualitatively the dynamics of local climate systems, but each local climate system can realize such possibility or can ignore it.

Next, if the HDS-hypothesis is conceptually true, then the specific regularities of $H D S$-dynamics should be concordant with those observed with local climate dynamics. These regularities can be built by the following characteristics of $A W C C$-units [Fig. 11(a)]: time coordinates (dmin and $d \max$ ) and temperature coordinates (tmin and tmax) of annual extremums; duration (period), averaged temperature (tmean), amplitude ( $a m p l)$. Among these characteristics only two notions (tmean and $a m p l)$ can be partly associated with the habitual notions of the uni-behavior conception. Why partly? Because these characteristics are calculated within $A W C C$-units but not within $A T V$-units [i.e. for temperature samplings which have some 
Probability of

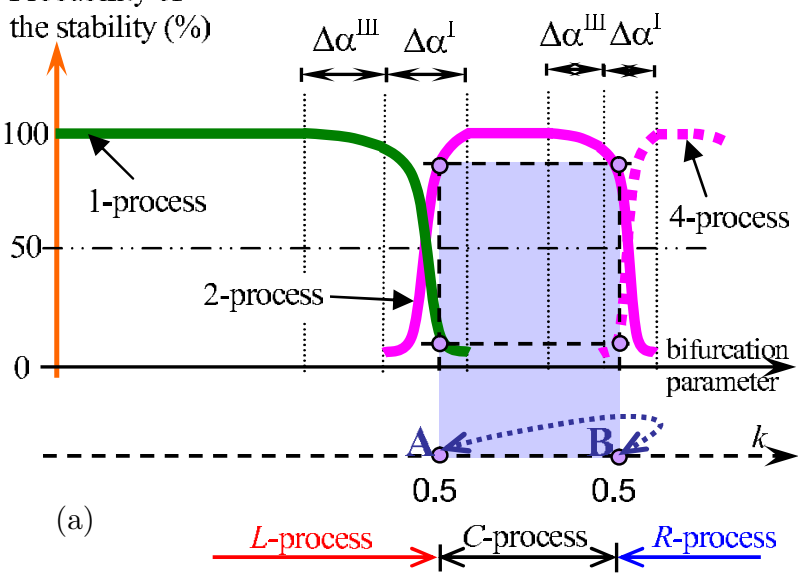

Probability of

the stability $(\%)$

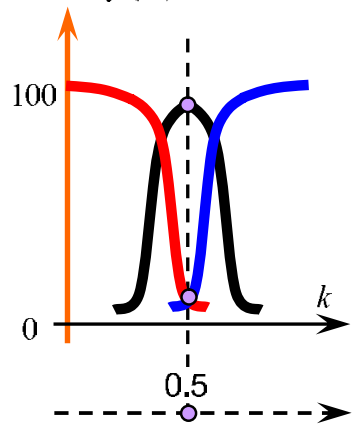

$C$-process

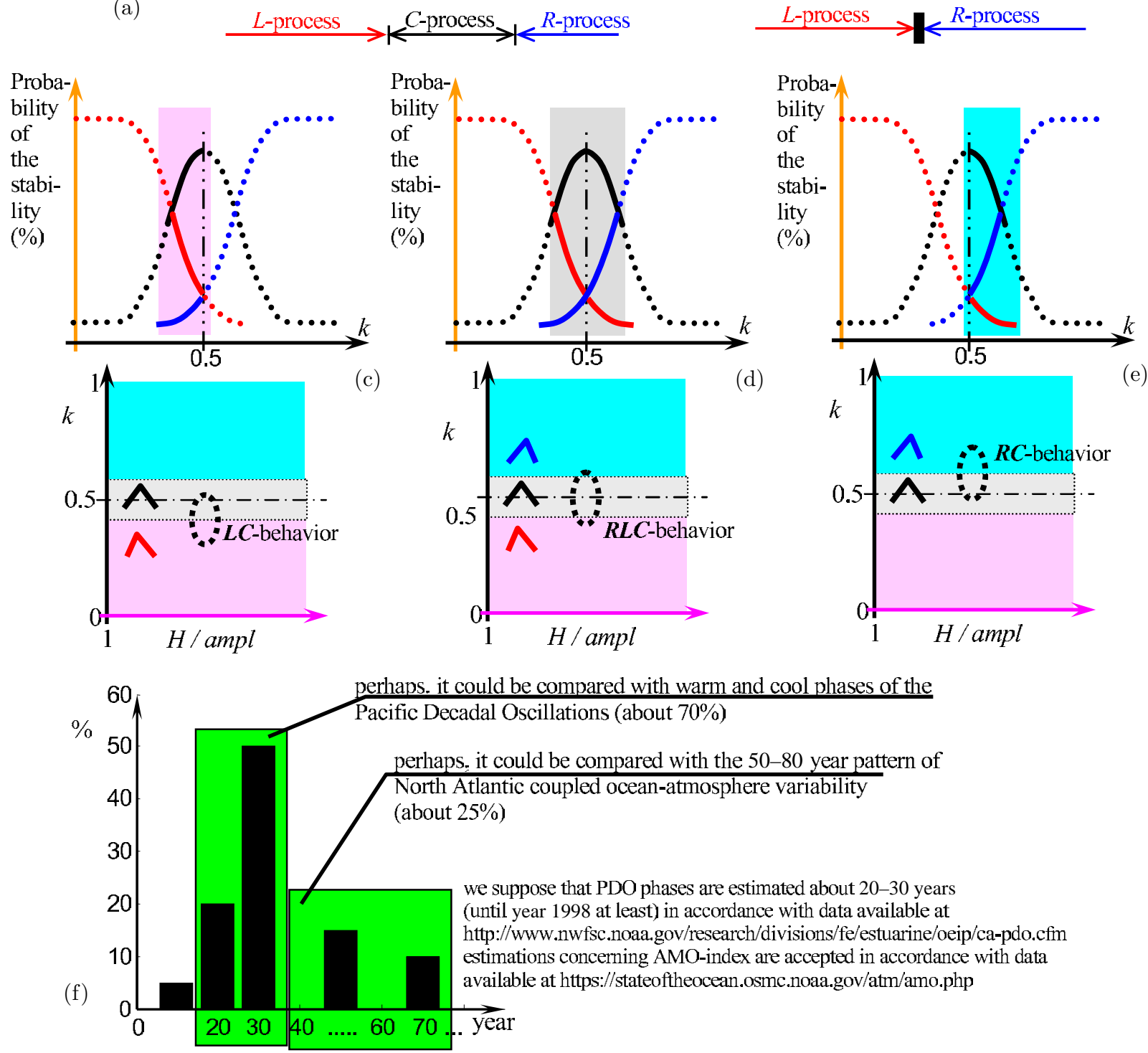

Fig. 15. Continued consideration of Fig. [12 d): (a) uncertainty zones about two sequential bifurcation points, adapted from Kolokolov \& Monovskaya, 2013a, 2013b]; (b) the result of the structural modification under the condition that the central part in Fig. 15]a) is reduced to the minimal range. (c)-(e) Particular views of the modified bifurcation diagram (upper parts) for $R C$-, $R L C$-, and $L C$-behaviors, correspondingly, combined with the baseline behavioral evolution in the parametrical space (low parts). (f) The distribution to the durations of $150 \mathrm{QH}$-sections, adapted from Kolokolov \& Monovskaya, 2016b]. 

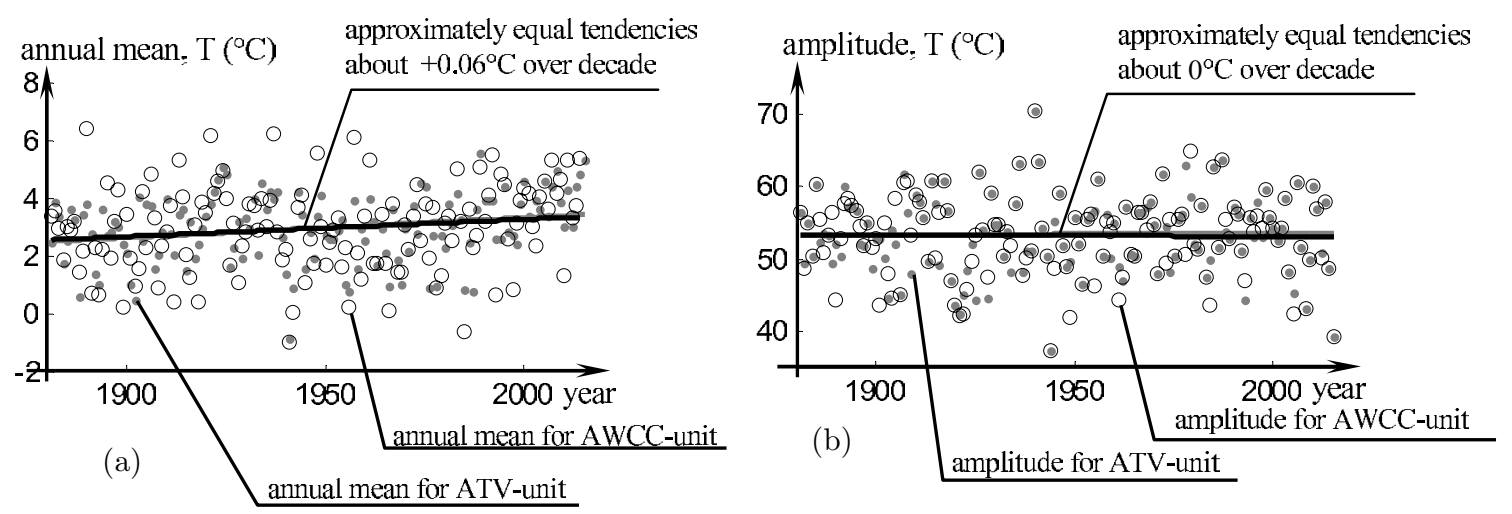

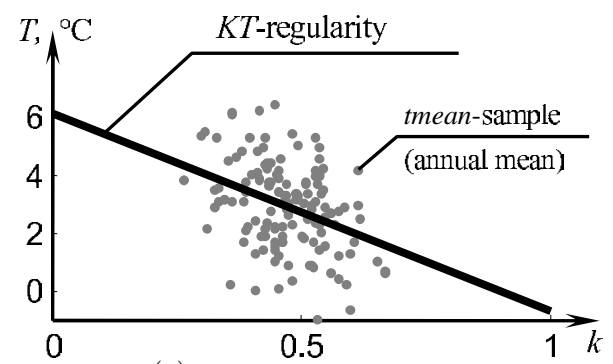

(c)
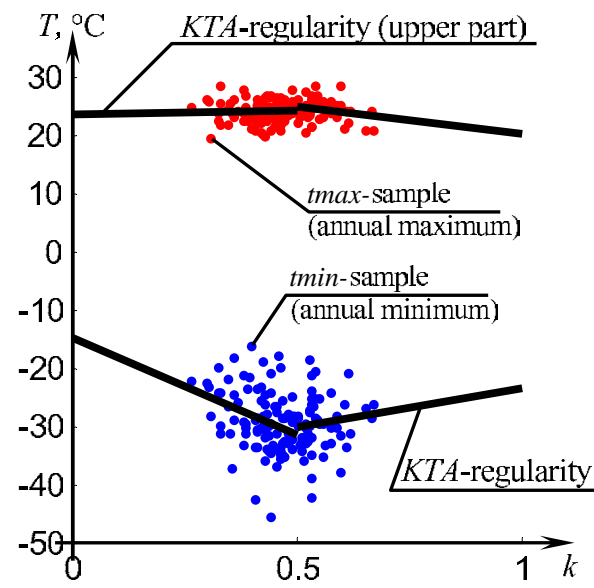
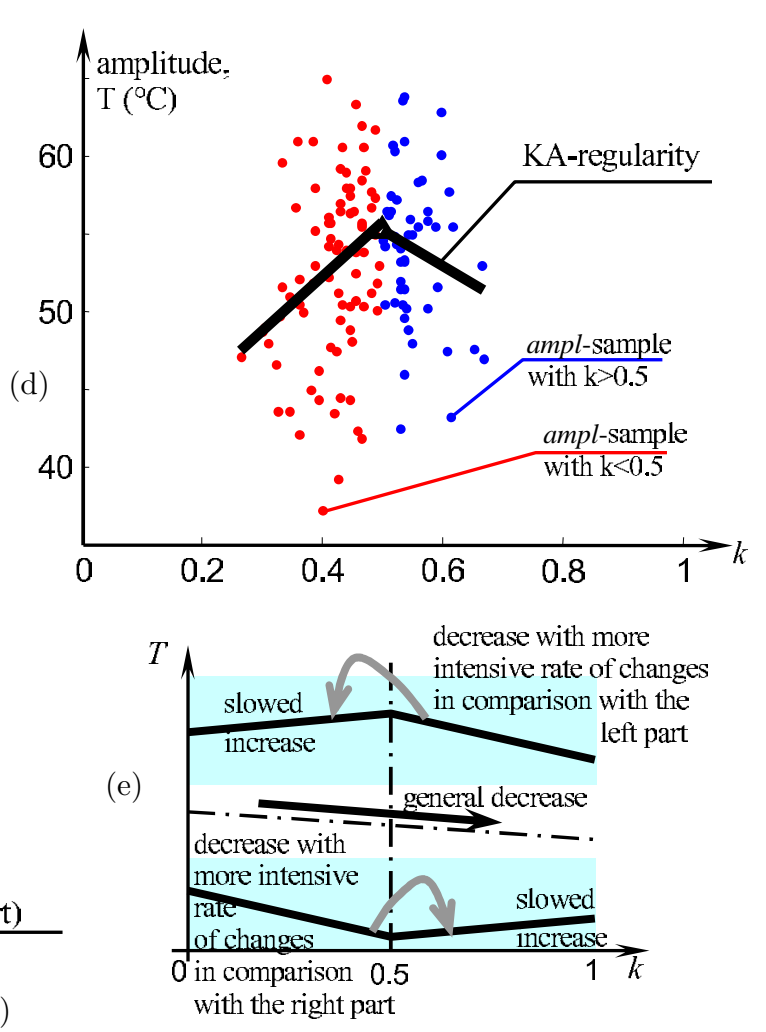

Fig. 16. (a) and (b) Tendencies built for Vutegra and St. Petersburg concerning annual data and annual amplitudes correspondingly. Results of the calculations made for Vutegra: (c) and (d) confirmations to a negative linear coefficient of $K T$ regularity and to two-sides view of $K A$-regularity correspondingly; (e) and (f) scheme and KTA-regularity confirming this scheme correspondingly.

differences described in Fig. 11(a) and compared in Figs. 6(d) and 6(e)]. Let us illustrate comparatively several typical cases in this connection [Figs. 16)(a) and 16(b)], namely: linear tendencies of temperature time series are usually similar, but particularities in rate and swing can be significantly different. The remaining characteristics ( $k$, period, dmin, $d \max )$ are not practically considered by the conventional methods.

So, similarities between notions from multiand uni-behavior conceptions are conditional, and this fact has forced us to introduce new notions. In particular, so-called $K T$-, $K A-, K P$ - and $K D$ regularities are used to show the arguments to verify the $H D S$-hypothesis. In accordance with $K T$ regularity, the averaged temperature of $R$-processes should be rather smaller than the averaged temperature of $L$-processes [Fig. 11(b)]; thus, the linear coefficient of tmean $(k)$-dependence determined by processing the temperature observations should be negative [Fig. 16)(c)]. In accordance with $K A$ regularity, the maximal amplitude should be with 
$k=0.5$, the minimal amplitudes should be with $k$ close to 0 and to 1 [Fig. 11(b)]; thus, $\operatorname{ampl}(k)$ dependence determined by processing the temperature observations should be described by bipartite symmetrical linear coefficients [Fig. [16(d)]. Next, $K T$ - and $K A$-regularities can together help to estimate local temperature variability. Details of such estimation (so-called KTA-regularity) are explained in Fig. 16(e), the result of the corresponding processing is shown in Fig. 16(f).

Peculiarities of time variability (Fig. 10) are beyond the uni-behavior conception. The HDShypothesis begins to fill this gap by $K D$ - and $K P$-regularities which visualize distinctive features caused by the alternation of the permitted passes through the synchronization sequences $\left[F_{1^{-}}\right.$and $F_{2}$-sequences, Fig. [1](b)]. We focus partly on the time interrelations between temperature maximums and minimums in Fig. 10(h), where the variability originates from the general diagram [Fig. 11(b)] according to the rule of the modes formulated in [Kolokolov \& Monovskaya, 2016a], namely [Fig. 10(h)]: the majority of dmin-values with $k>0.5$ (blue points) are on the left from the majority of $d m i n$-values with $k<0.5$ (red points); the majority of $d$ max-values with $k>0.5$ (blue points) are on the right from the majority of $d$ maxvalues with $k<0.5$ (red points). Generally, $K D$ regularity is decomposed into two bipartite asymmetrical parts built for dmin- and dmax-tendencies [Fig. 17(a)], where a distance between these parts with $k=0.5$ is about a half of a year. The $K P$ regularity is caused by the asymmetrical structural changes during transients from one elementary process to the other [Figs. 17(b) and [17)(c)]. The corresponding asymmetry of the averaged period-value means that the linear coefficient of period/year $(k)$ dependence should be positive [Fig. 17(d)]. The following confirmations occur currently: $100 \%$ cases for $K D$-, $K T$-, and $K P$-regularities; $93 \%$ cases for $K A$-and $K T A$-regularities.

Next, let us clarify the moment that currently remains beyond the consideration. It is the point of the fourth behavior [Fig. 10(a), black dotted line], viz. if the $H D S$-hypothesis is conceptually true, then why do we consider the disposition of only three elementary processes [Fig.[11(b)]? Briefly, the answer consists of the following: the fourth behavior can occur [Fig. 18(a)], but its stable realization is highly unlikely Kolokolov \& Monovskava, 2015f]. Here, let us comment on the main points. Let us denote the fourth process as $C^{*}$-process with $k=$ 0.5, where the phase point passes through 3-2-1-43 -surfaces [Fig. [18(a)]. Then disposition of $R-, L-$, $C-, C^{*}$-processes can be visually explained in $\left(D_{\text {rel }}\right.$, $k$ )-space [Fig. [18(b)], where $D_{\text {rel }}=d \min / 0.5 T_{S}$. In other words, $C^{*}$-process can be formally located as mentioned in Fig. 10(a); however, its stable realization can occur only with $\mathrm{H} / \mathrm{ampl}=1$. While applying to the local climate dynamics, it can be concluded as follows: the complete ensemble of four elementary processes with the same periodicity (close to year) and different orders of structural changes can occur with $H / a m p l$ close to 1 ; and this supposes that the temperature variability is practically absent [like in Fig. 10(c)]. So, the $H D S$ hypothesis includes all the potentially possible dispositions; however, $C^{*}$-process is realized as either a rare particular case or as transient disturbances restricted within a triangle-like domain [Fig. 18(c)].

Intermittency effects demand to pay attention to transients. Let us mention, that reconstructions made by processing the temperature observations show that $\mathrm{H} / \mathrm{ampl}$-values are approximately from 1.1 to 1.9 with $k=0.2 \ldots 0.8$ Kolokolov \& Monovskava, 2015b, 2016a. And the more the $H / a m p l$-value is, the farther are the transitions between $R$-, $L$ - and $C$-processes [Fig. 13(d) in comparison with Fig. 13(e)]. Two mechanisms provide these transitions Kolokolov et al., 2003; Kolokolov \& Monovskaya, 2015f, namely: 32143mechanism means transitions "by the traces" of 32143-structure [Figs. 18(d) and 18(f)]; 242mechanism means transitions "by the traces" of 242-structure [Figs. 18)(e) and 18(g)]. The peculiarities of the 242-mechanism consist of the following: the duration of each fragment is equal to the synchronization period; the amplitude of each fragment is approximately permanent; the averaged-per-period value gradually changes. The peculiarities of the 32143-mechanism consist of the following: the duration of each fragment is more than the synchronization period; the amplitude of each fragment is more than the forthcoming periodical process; and the averaged-per-period value is approximately permanent. Thus, the structures of both $C$ - and $C^{*}$-processes facilitate transients between the elementary processes; however, 242structure is universal and 32143-structure accompanies mainly $L$-process [Fig. 18(c)].

The presented summary is based on the results made for the long-term temperature observations 

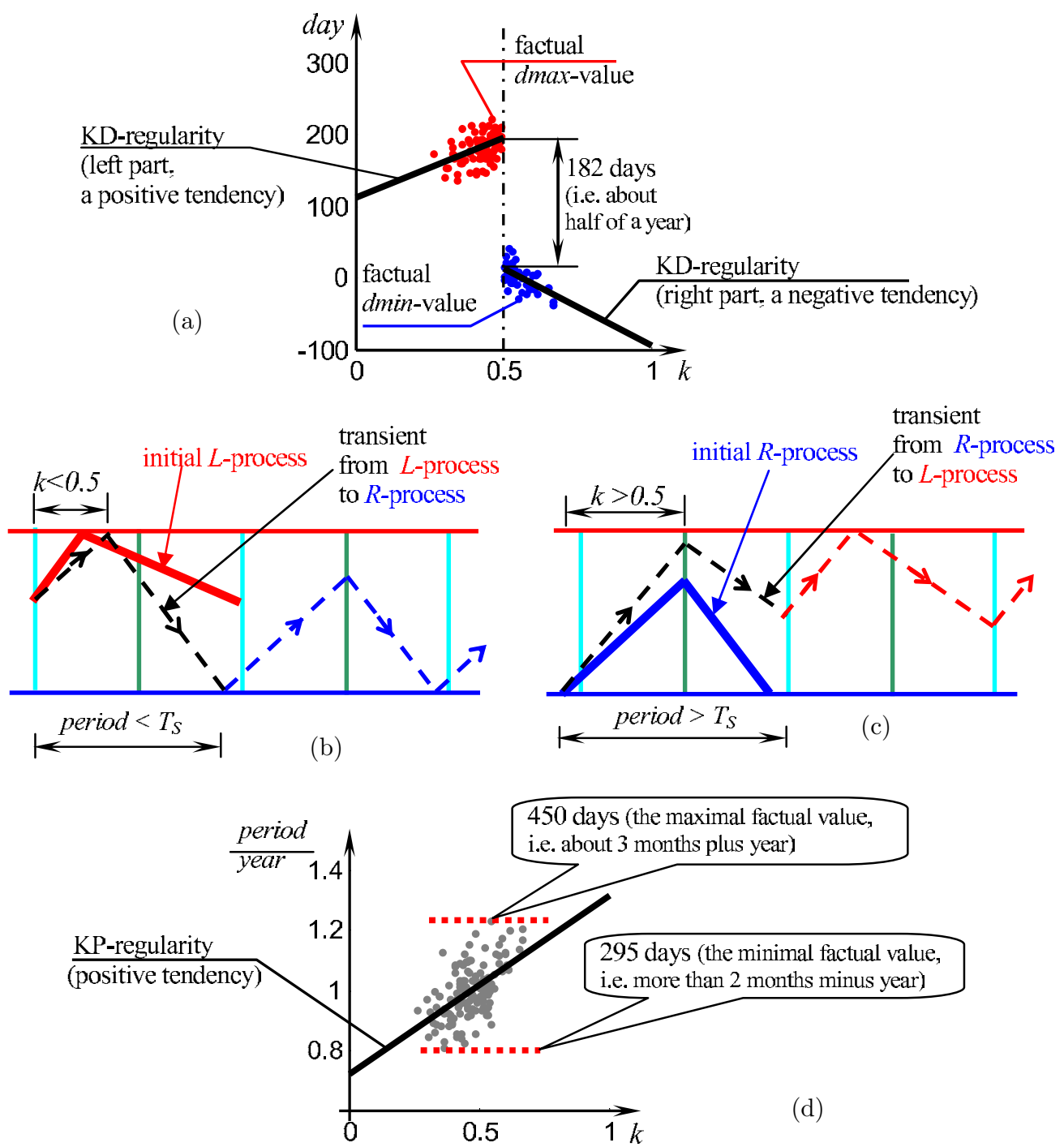

Fig. 17. (a) Confirmation of two-sides KD-regularity. (b) and (c) Schemes to explain the specific mechanisms on how variation of annual warming-cooling cycles is formed in accordance with the $H D S$-model taking into account the alternation of the permitted transitions through the synchronization surfaces [Fig. [1]b)]. (d) Confirmation of negative KP-regularity. Results presented in (a) and (d) are calculated for Vutegra.

in Russia. With some assumptions, the HDShypothesis includes the traditional uni-behavior viewpoint as a particular case due to following facts: one of elementary processes (mainly $C$-behavior) dominates usually among others that can look like a uni-behavior mode [Figs. 12(f), 13(a), 13(b) and 14(c)]; local evolutional cycles of 30-years long dominate among other cycles [Fig. 15(f)]; comparatively weak alternations of several constituents can look like the habitual and quite stable unibehavior mode accompanied by chaotic fluctuations [Fig. 13(d)]. However, explanations concerning the observed oddities suppose the general (multibehavior) viewpoint provided by the $H D S$-hypothesis. And then, bifurcations in the dynamics of local climate systems exist and determine typical local behaviors over the last 135 years at least. Both order and activities of bifurcations are different for local climate systems; however, the regularities of dynamics caused by the $H D S$-law are general for all local climate systems. Mainly, three periodical processes with the same period (1-year) and different annual temperature patterns are observed. These differences bring distortions to the results of statistical and time-and-frequency processing (first of all, multimodality effects). Bifurcations of period-doubling are formally possible in the $H D S$ dynamics, for example Kolokolov et al., 2003]; however, the corresponding parametrical conditions are not yet identified for the time being. 

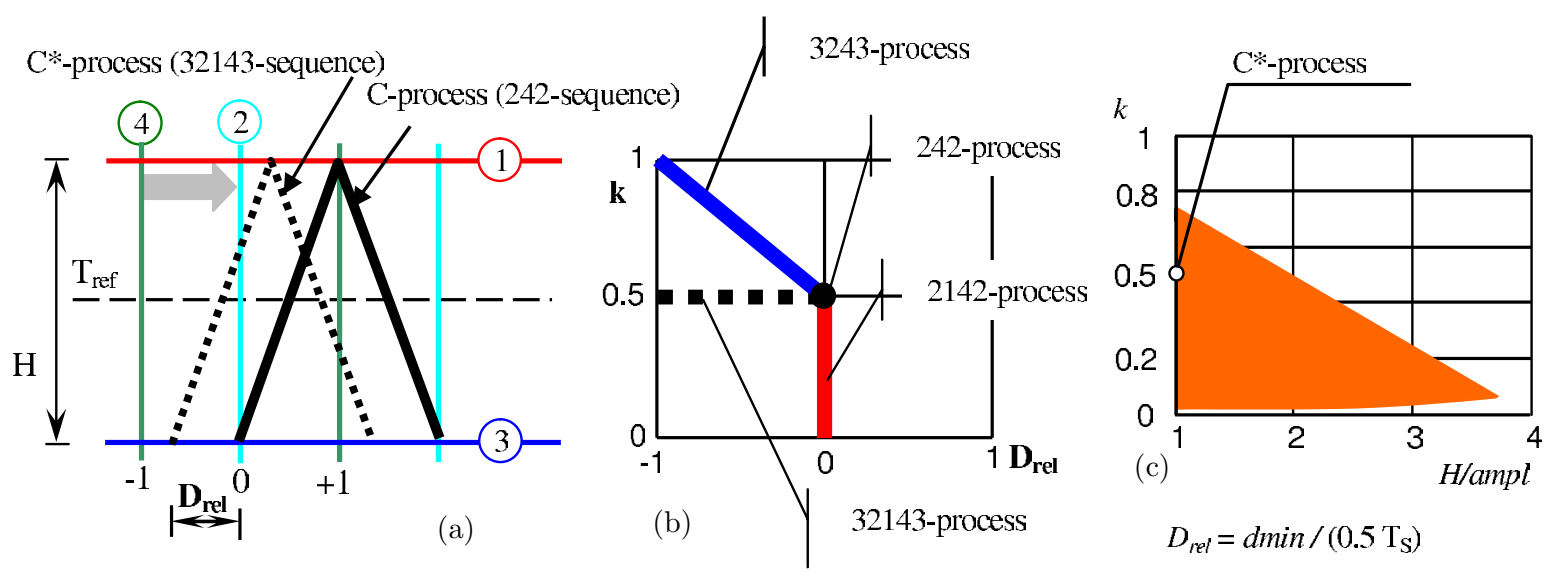

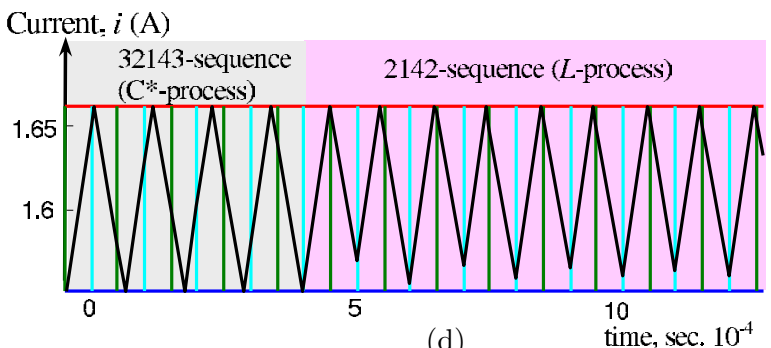

(d)

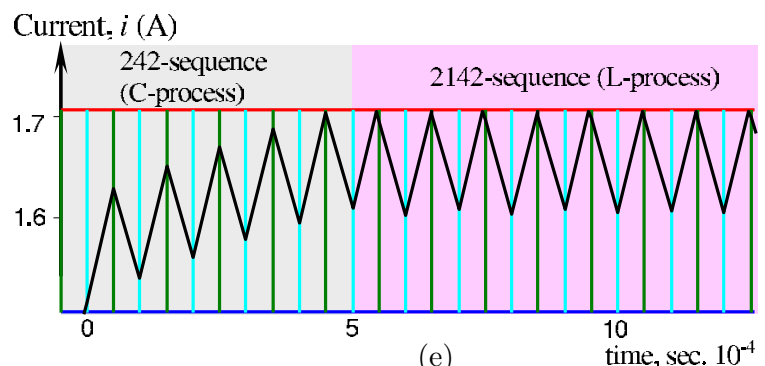

Current. $i$ (A)

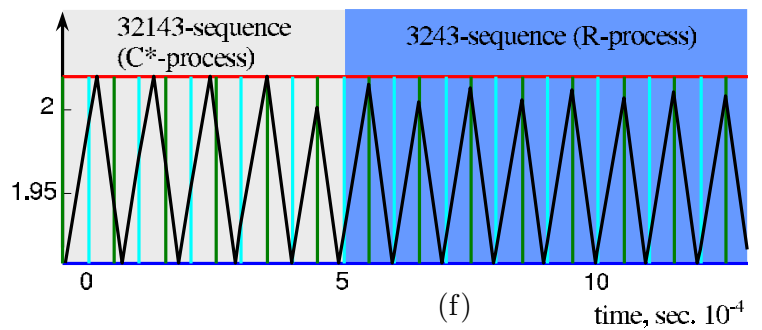

Current, $i$ (A)

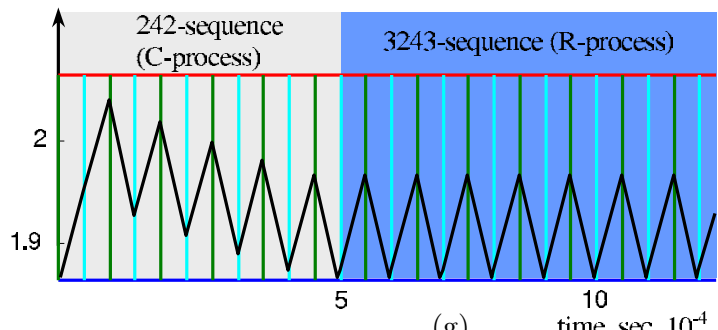

(g)

Fig. 18. (a) and (b) Supplementary diagrams of Fig. 11(b) to illustrate the fourth elementary process $\left(C^{*}\right.$-process) in comparison with $C$-process taking into account the alternation of the permitted transitions through the synchronization surfaces and together with $R L C$-ensemble in $\left(D_{\text {rel }}, k\right)$-space correspondingly. Results of model-based simulations of $H D S$-regulator dynamics to comment on $C^{*}$-process peculiarities: (c) domain of influence of $C^{*}$-process on transients in $(H / a m p l, k)$-space; (d) and (e) convergence towards 2142-process ( $L$-process) "by the traces" of 32143 -sequence $\left(C^{*}\right.$-process) and 242 -sequence ( $C$-process) correspondingly; (f) and (g) convergence towards 3243-process $\left(R\right.$-process) "by the traces" of 32143-sequence $\left(C^{*}\right.$ process) and 242-sequence ( $C$-process) correspondingly. Figures (a)-(g) are adapted from [Kolokolov \& Monovskava, 2015f].

\section{A Novel Statement on the Stability Margin Towards Emergency}

So, in accordance with the $H D S$-model, a typical behavior of local climate system represents alternations between three elementary processes. Such behavior obeys all the peculiar regularities of the $H D S$-dynamics $(K T$-, $K A$-, $K D$-, $K P$-regularities and so on) and provides stable periodicity (about 1 year) in wide ranges of internal and external parametric variation; so, it resembles only outwardly chaotic manner. From the admission that the HDShypothesis is conceptually true, a way to visualize the local dynamics evolution based on processing the temperature observations over the last century in terms of the bifurcation analysis was first proposed in Kolokolov \& Monovskava, 2015a], where the logically consistent explanation of mechanisms of the interannual variability was described by a dynamical system. Yes, it demands modifications of the habitual viewpoint regarding local evolution; however, we are sure that it is inevitable to understand and forecast emergencies in local climate dynamics. In this paper, we begin to discuss problems of emergency with the HDS-model.

Then local climate dynamics is generally described using the ensemble terms [i.e. as several 
interrelated behaviors, Fig. [19(a)] within the moving coordinate system [denoted by two-way arrows in Fig. 19](a)], where each local realization over time has its own evolutional pattern [for example, Figs. 12(f), 13(a), 13(b) and 14(c)]. Despite the complexity of such dynamics, it is possible to decompose this into conditionally independent constituents. Let us summarize and comment on these constituents in comparison with the traditional viewpoint on local climate dynamics. Let us continue to use the local dynamics observed in Vutegra to illustrate. So, usually, dynamics evolution is realized as a sequence of transitions about $k=0.5$ from one side to the other and vice versa [for example, between A- and B-sections, Fig. [19(b)]. Beginning dates and durations of these transitions determine the time windows of quasi-homogeneous dynamics ( $Q H$-sections) for each local climate system. Correspondingly, patterns of $Q H$-sections for several local climate systems are different to a more or less extent, for example Kolokolov \& Monovskava, 2015a, 2015b, 2016a, 2016b], in contrast to the traditional conception, where time windows are unified in the beginning dates for a constant duration for all local climate systems. For example, the patterns of local evolution in Vutegra [Fig. 14(c)] and in Alexandrov-Sahalinsky [initial diagram used for Fig. [14(f)] are described by obviously different $Q H$ sections.

Hereafter, the variation of the main bifurcation parameter $(k)$ is considered, and the remaining potentially variable parameters are supposed to be rather invariable. An $R L C$-ensemble is determined for each $Q H$-section Kolokolov \& Monovskava, 2016b, 2016c], where each of the behaviors has several possible realizations in accordance with the identified range of $k$-variation. So, each behavior occupies a domain in the time-and-temperature space. For example, $($ day, $T)$-domains of $R-, L-, C$ behaviors are denoted by azure, pink and gray colors correspondingly in Fig. 19(c). In other words, local dynamics during a calendar year is not homogeneous and should be described by several behaviors in contrast to the traditional conception, where one averaged annual behavior is supposed, for example, see Figs. 4(e), 10(b) and 10(d). In particular, local climate dynamics over the last century in Russia should be described by three behaviors $(R-$, $L$-, $C$-behaviors) at least, for example [Kolokolov \& Monovskaya, 2016a]. In some circumstances, differences between these behaviors can be comparatively small [Fig. 13(d)], and three behaviors can look like one behavior with fluctuations close to white noise; however, the nonlinear nature of the differences assists permanently and brings latent emergency (see below, Fig. 21). Thus, a calendar day is a heterogeneous notion, because each daily means is related to several (day, T)-domains [Fig. [19(d)]. So, the traditional view on homogeneous daily means relates rather to the habitual thinking.

Next, the traditional viewpoint on local climate dynamics pays essentially more attention to temperature in comparison with time. In particular, days of annual temperature extremes are practically excluded from detailed considerations; and the description of factual annual minimums can be even interrupted by beginnings of year [Figs. 6(b), 6(c), 6(g) and 6(h)]. In this connection, the $H D S$-model uses fragments of annual warmingcooling cycles ( $A W C C$-units) in contrast to fragments of annual temperature variation ( $A T V$-units) restricted by a calendar year [Fig. 19)(e)]. Nevertheless, the structure of $R L C$-ensemble in $A W C C$-unit terms [Fig. 19(c)] can be reduced to a calendar year [Fig. 19(d)], where limits of the potentialities can be contoured by a contour-line of the $R L C$-domain [violet dotted contour-line in Fig. [19(d)]. Then, it becomes possible to compare the descriptions of the temperature observations based on $R L C$-ensembles [for example, Fig. 19.(c)] with the ones based on the traditional viewpoint. Namely, monthly means in accordance with the local climate norms [12 bright green steps in Fig. 19(e)] overlap only part of the factual realizations [triangle-like fragments in Fig. 19(e)]; contour-line of $R L C$-domain covers practically all factual realizations [violet bold dotted contours in Fig. 19(f)]. Thus the following conclusion exists: such covering means that the physical nature of the observed annual uncertainty is not chaotic, and this nature can be determined by clear cause-effect relations based on regular evolution of the elementary processes with parametric variation.

Per se, these cause-effect relations resolve conflict-of-units between notions used to understand natural evolution processes and notions used to describe desirable artificial regimes [Kolokolov \& Monovskaya, 2016b, 2016c, 2018]. Here natural evolution processes are connected with the dynamics of the elementary processes; and desirable artificial regimes are connected with deviations of some habitual local climate due to human habits and 


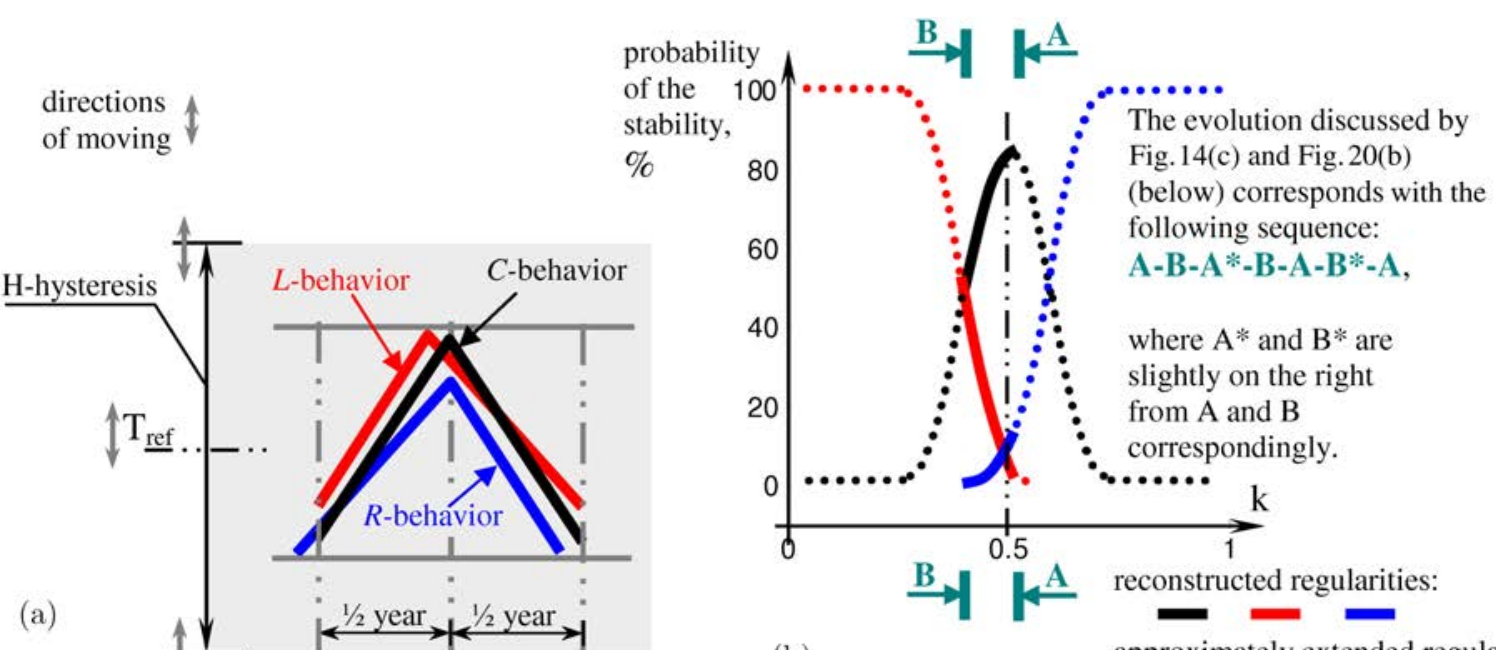

(a)

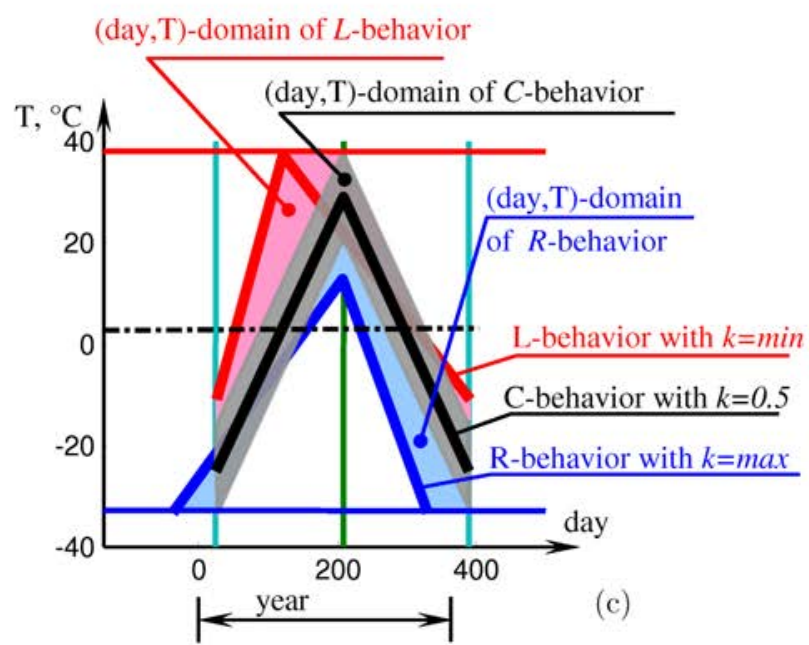

(d)
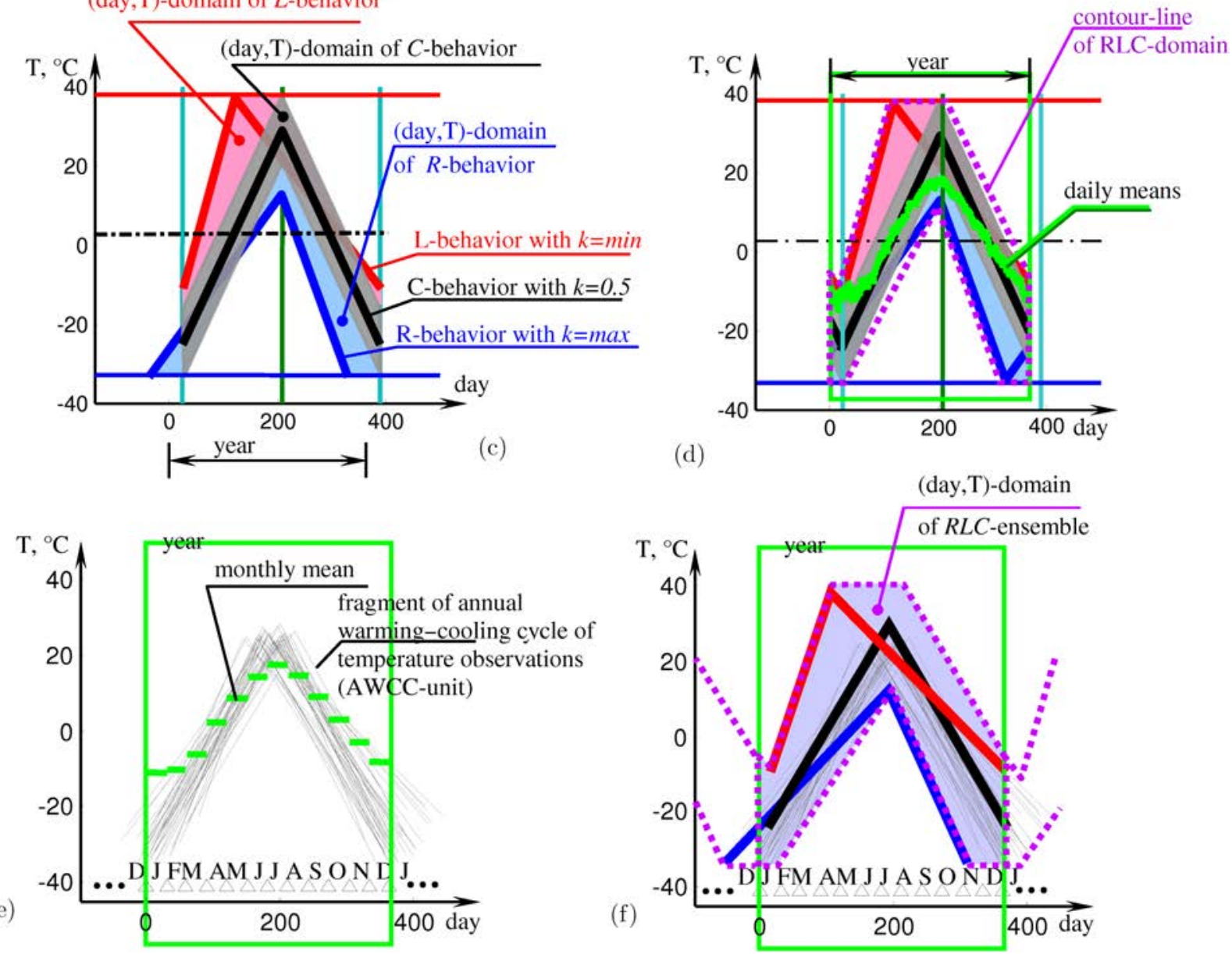

(f)

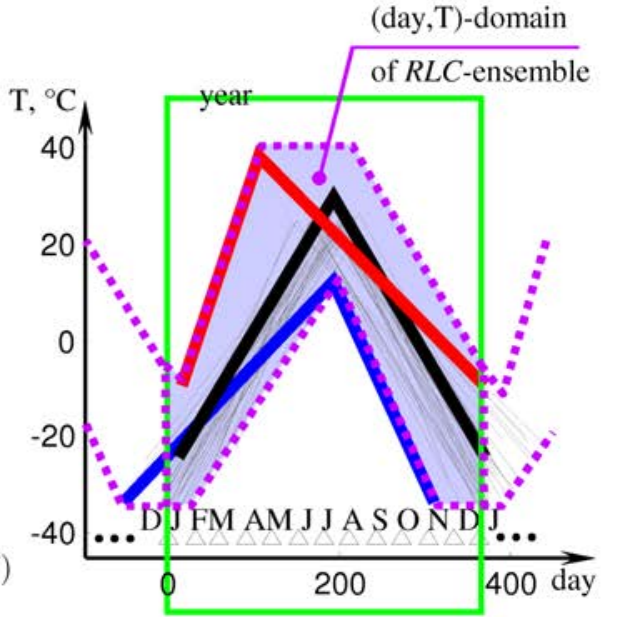

Fig. 19. (a) Scheme of $R L C$-ensemble within the moving $\left(T_{\text {ref }}, H\right)$-coordinates. Results of calculations by processing the temperature observations in Vutegra over the first $Q H$-section [Fig. 14(c)]: (b) diagram of existence of $R$-, $L$-, $C$-processes in the probable sense with $k$-variation; (c) and (d) domains of $R$-, $L$-, $C$-behaviors in (day, $T$ )-space in $A W C C$-unit terms and reduced to $A T V$-unit terms correspondingly; (e) and (f) fragments of annual warming-cooling cycles in (day, $T$ )-space combined with monthly means and the domain of $R L C$-ensemble correspondingly. 
desires. For example, it was already mentioned (Sec. 2) that an annual averaged temperature is the most widespread variable used in estimations on climate changes. Let us illustrate the cause-effect relations from this variable. And let us accept that an annual temperature averaged per year is approximately equal to the one averaged per fragment [i.e. to tmean-value, Fig. 11(a)] because their tendencies approximately coincide [Fig. 16(a)]. Taking into account $K T$-regularity [Fig. [16(c)], each bifurcation between the elementary processes leads to tmeanvariation more or less [Fig. 20(a)]: generally, it becomes comparatively warmer towards $L$-behavior and comparatively colder towards $R$-behavior. In the evolutionary terms of the intermittency, this means transitions during quasi-homogeneous sections [Figs. [20(b) and [19(b)]. However, here the constancy of the main parameters of the $H D S$-model

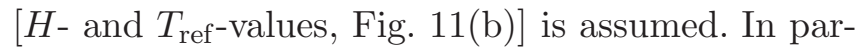
ticular, calculations presented in Figs. 19(c)-19(f) were done for the first $Q H$-section [Fig. 20(b)], within which such constancy is observed. Otherwise (i.e. with variation of $H$ - and/or $T_{\text {ref }^{-}}$ parameters), the system of coordinates, into which $R L C$-ensemble is encapsulated, moves [Fig. [19(a)].

Let the moving occur. For example, let us consider a mechanism of $T_{\text {ref-variation [Fig. 20(c)] }}$. Let a local climate system be characterized by the comparatively colder behavior within its local context [i.e. it is $R$-behavior in relation to the initial

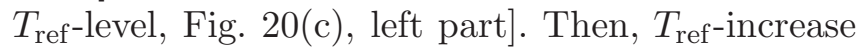
leads to increase in annual means for any behavior within the $R L C$-ensemble [Fig. 20(c), right part], including the rise in temperature for the coldest behavior. And, vice versa, $T_{\text {ref-decrease leads to }}$ general decrease in annual means within the $R L C$ ensemble, including the fall of temperature for the warmest behavior. In other words, from the $H D S$ model, $T_{\text {ref-variation represents one of the causes for }}$ changes of annual means. At the same time, effects of $T_{\text {ref-variation can be compensated by concurrent }}$ bifurcations within the $R L C$-ensemble. For example, let initially $L$-behavior occur. Let $T_{\text {ref-increase }}$ occur with a bifurcation from $L$-behavior to $C$ behavior [red solid broken line in Fig. 20(c)]. Then the fall of temperature can be realized under the

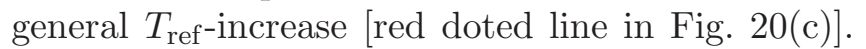
So, tendencies calculated for annual means and $T_{\text {ref- }}-$ variation are different due to the superficial effect and the intrinsic cause correspondingly, for example, see Fig. 20(d). In comparison with the modified bifurcation diagram [Fig. 20(b)], let us mention that the essential changes of parameters of the moving system of coordinates can occur independently of the bifurcations. In particular, the crucial (in a centennial scale) $T_{\text {ref-change occurs within the }}$ second $Q H$-section [Fig. 20(d)]. Since such crucial event is not a bifurcation, then we use the notion of a change-point to denote this [Kolokolov \& Monovskaya, 2017a].

Similar consideration can be made to $H$ variation [scheme in Fig. 20(e)]: the more $H$-value changes, the more annual means vary without bifurcation phenomena; and such variation is asymmetrical. For example, $H$-decrease [Fig. 20(e), right part in comparison with left part] leads to increase in annual means for comparatively cold states and to decrease in annual means for comparatively warm states within $R L C$-ensemble; and $H$-increase returns vice versa. The mechanism of $H$-variation can also generate change-points. For example, the sequence of the approximated $H$-tendencies is presented in Fig. 20(f), where two change-points occur within the second $Q H$-section and two changepoints occur within the third $Q H$-section. The discussed three mechanisms of variation of annual means [mechanisms of $C$-bifurcation, $T_{\text {ref-variation, }}$ $H$-variation, in Figs. 20(a), 20(c) and 20(e) correspondingly] can occur independently or jointly in various combinations. And, in addition to annual means, let us mention two other superficial characteristics to describe annual warming-cooling cycles, namely: frequency and shape. Why is that necessary to discuss? Because a change of one of the temperature characteristics can be initiated by several physical causes; and, vice versa, one physical cause can lead to changes of several temperature characteristics [for example, Fig. 20(g)].

Let us use the general ideology proposed within the bifurcation-fractal analytics in order to find conflict-free solutions in similar situations for other systems with variable structures [Kolokolov \& Monovskaya, 2018]. Then the analytics includes the following main subjects: a typical (normal) behavior, a stability margin, an emergency. The corresponding images should be built into the structure of dynamics evolution in the parametrical space. Here the similarity with technical realizations of $H D S$-regulators could help to illustrate the essence of consideration. Such regulators are designed to guarantee the process of energy conversion with a stable synchronization frequency over the full range 


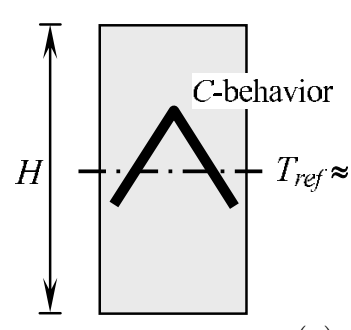

(a)
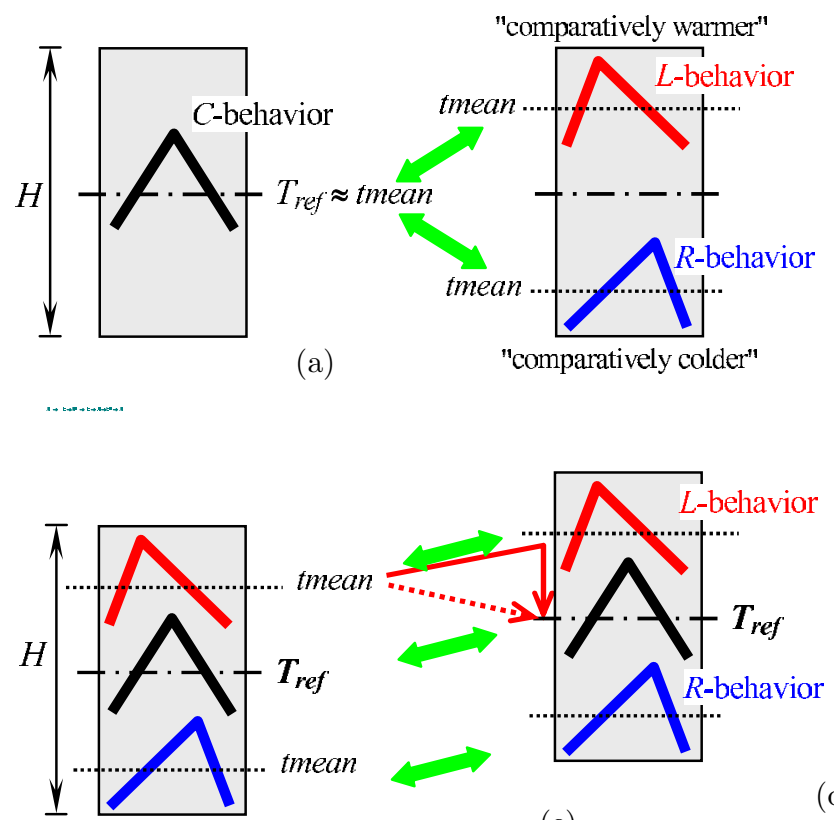

(c)
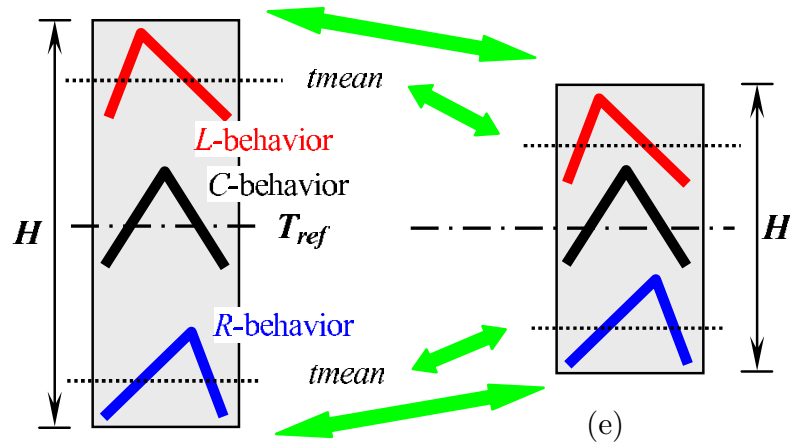

(e) probability of the stability, $\%$

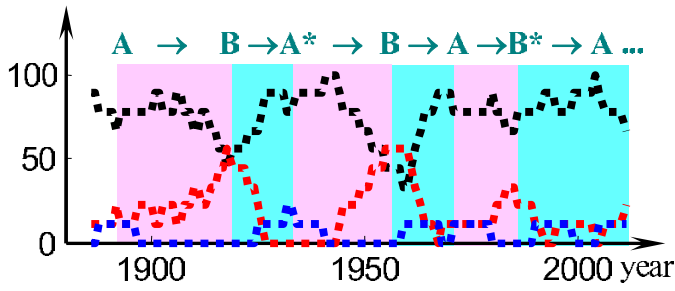

(b)

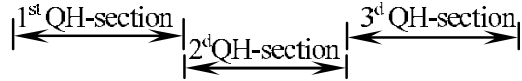

(d)
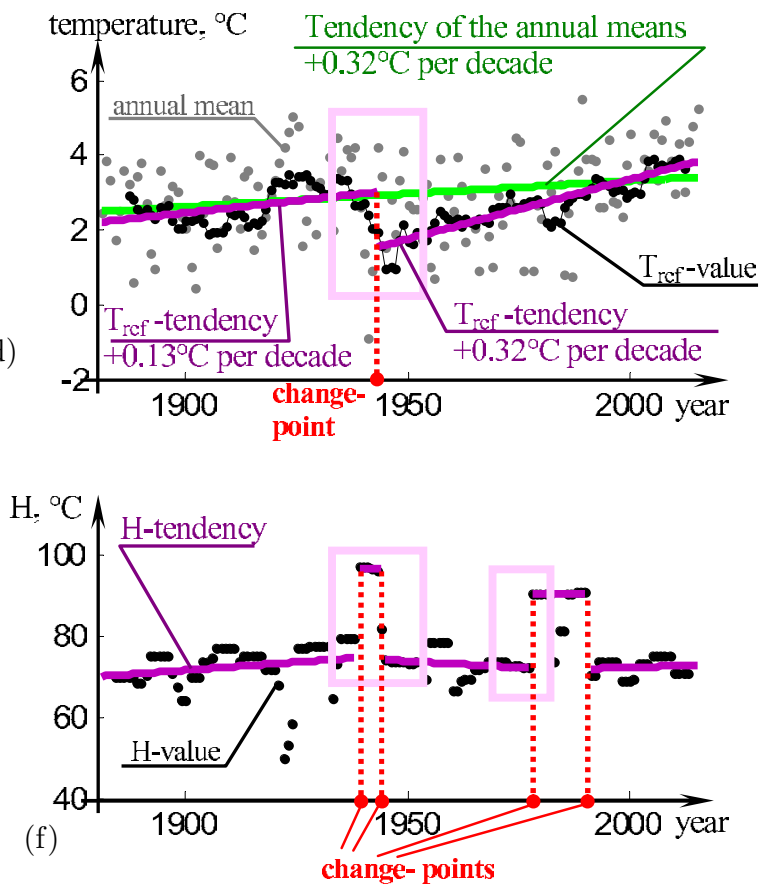

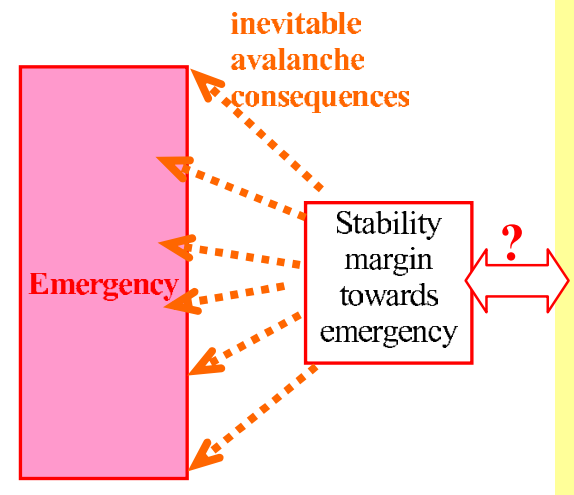

(h)

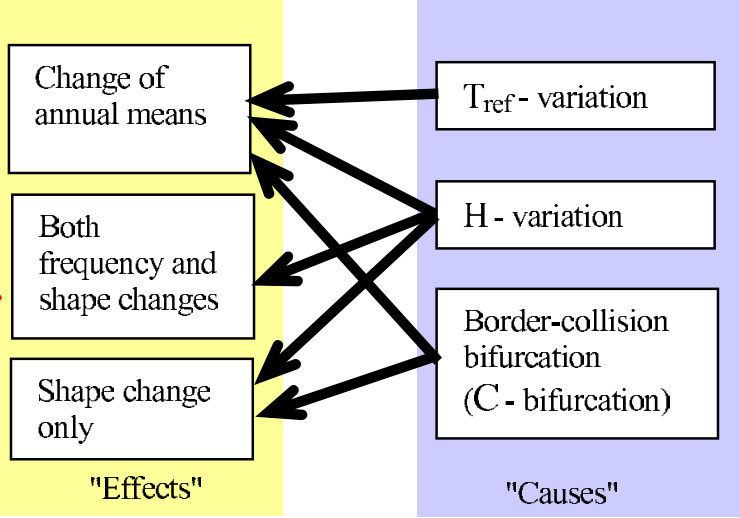

$(\mathrm{g})$

Fig. 20. (a), (c), (e) and (b), (d), (f) Schemes and examples to illustrate mechanisms of temperature evolution in accordance with the HDS-model correspondingly. The calculations are made for Vutegra, where Fig. 20 b) continues the consideration on the modified bifurcation diagram presented in Figs. 14 c) and 19 b). (g) and (h) Comments on cause-effect relations and on emergency, correspondingly, made in the context of the mechanisms of temperature evolution. 
of a duty cycle in contrast to synchronous one-level regulators which can only partly provide the stability, for example [Zhou et al., 2012]. Results of the bifurcation analysis of $H D S$-regulator dynamics, for example Kolokolov et al. . 2003: Kolokolov \& Monovskaya, 2015e, 2015f], show that processes with frequencies unequal to the synchronization frequency $\left(1 / T_{S}\right)$ are located within the semicircle on the left of the point $(1,0.5)$ in $(H / a m p l, k)$-space [black bold dotted curve in Fig. 21(a)]. Frequency changes are inadmissible in operation due to it means emergency. At the same time, there are other characteristics of the operating performance which are desirable to increase the efficiency. As a result, there is the so-called stability margin unit (a minimal margin towards emergency) which provides this demand in order to separate abnormal processes from the operating regime [Fig. 21(a), upper part].

Let us suppose that the habitual annual temperature variation observed in a local climate system over the last century (i.e. a typical behavior) is similar to an operating regime in an artificial system. Then, let us determine the domains of the typical and atypical behaviors in $(H / a m p l, k)$ space. Let us take into account that destructions of annual seasonality as well as too large oscillations of annual means will lead to events which are rather undesirable in human activities. So, atypical
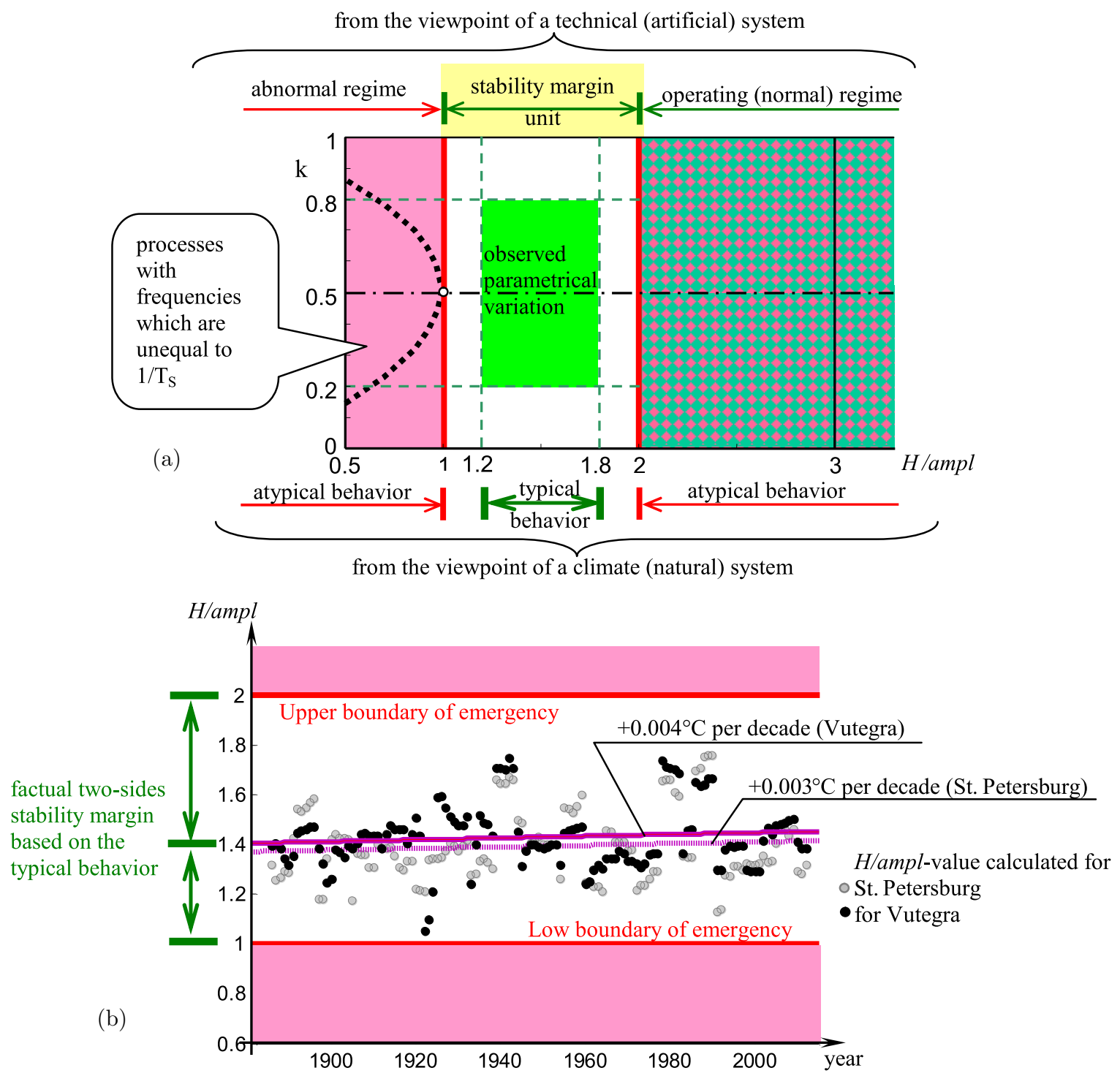

Fig. 21. (a) Domains of desirable/undesirable dynamics in $(H / a m p l, k)$-space built for nonlinear systems under $H D S$-control from the artificial and natural viewpoints and (b) estimations of temperature stability margins concerning two emergency boundaries for Vutegra and St. Petersburg. 
behaviors exist on the left from $H / a m p l=1$ and on the right from $H / a m p l=2$ [Fig. 21](a), lower part]. Next, parametrical variation observed for the typical behavior is restricted within the following ranges: $0.2<k<0.8$ and $1.2<\mathrm{H} / \mathrm{ampl}<1.8$ Kolokolov \& Monovskava, 2015b, 2016a]. So, at present, the domain of interest for natural realizations is located with $1<H / a m p l<2$. And tendencies of dynamics evolution in this sense can be estimated for each local climate system in $(\mathrm{H} / \mathrm{ampl}$, time)-space. For example, $\mathrm{H} / \mathrm{ampl}$-tendencies calculated for St. Petersburg and Vutegra over time are shown in Fig. 21(b). These results demonstrate quite stable centerlines of the local evolution together with abrupt short-term deviations towards both boundaries; and such deviations become more frequent. We believe that transitions through the emergency boundaries in both directions can be realized like a shot. And it seems to be a natural feature of the $H D S$-dynamics parallel with the ability of the guaranteed stability of 1-year periodicity at the expense of the intermittency effects discussed above.

Let us pay attention to the fact that causes of the observed temperature changes due to abrupt shifts (so-called change-points) identified with variation of the $H D S$-regulator parameters [for example,

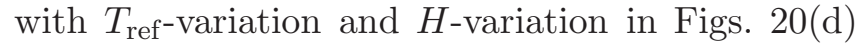
and [20(f) correspondingly] are distinguished from the bifurcations. The notion of a stability margin originates from the control theory and, in a wide sense, denotes a distance to an undesirable regime or to emergency. The bifurcation theory connects this notion with the distance to a bifurcation boundary in a parametrical space. So, in spite of mechanisms of temperature changes with/without bifurcations are different [Figs. 20(a), 20(c) and[20(e)], their conflict-free sewing becomes conceptually possible in the context of the stability margin towards emergencies determined from the bifurcation diagrams built in the parametrical space [Fig. 21(a)].

\section{Concluding Discussion}

This paper continues the discussion on the novel viewpoint on local climate dynamics (so-called HDS-hypothesis). Sections 2 and 3 show that the traditional (uni-behavior) approach to describe local climate dynamics needs serious modifications due to the contemporary challenges; Secs. 4 and 5 show that the developed (multibehavior) approach is quite justified from the bifurcation analysis conceptions. Section [6 presents a novel view on the emergency forecasting which becomes possible from the practice-oriented bifurcation analysis. Perhaps, at first glance, each of the main discussed constituents (i.e. the conceptual model, typical nonlinear scenarios, and used analytics) can look too unusual in the considered application. We are sure that it is just the subjective impression related to habitual thinking. And, perhaps, a deep gap between the notions of weather and climate is retained until now due to the research limits being artificially and groundlessly constricted.

We use the integrating analytics [Kolokolov \& Monovskaya, 2015c, 2016b, 2016c, 2017a, 2017b, 2017c, 2018] intended to resolve the so-called conflict-of-units between the notions used to comprehend natural evolution scenarios (for example, evolution of local climate dynamics) and the notions used to describe desirable behaviors (for example, habitual temperature patterns of local climate), where causes (changes in the stability degradation) and effects (changes in the observed characteristics) are conflict-free sewed [for example, Figs. 20(g) and 20(h)] in order to estimate the coming emergency level from the bifurcation analysis conceptions. Tools of such analytics demonstrate promising results in the application to artificial energy converters, for example Kolokolov \& Monovskaya, 2006, 2013b, 2018]. We translate these tools for similar problem (i.e. for emergency forecasting during transients caused by intermittency-like phenomena) when applying to a similar nonlinear system (i.e. to a system with a variable structure). Next, we analyze the intermittency on the basis of the modified bifurcation diagrams built as per the method of the uncertainty zones proposed and experimentally verified in [Kolokolov \& Monovskaya, 2013a]. We are forced to use the modified bifurcation diagrams because the classic bifurcation diagrams are not intended for it, especially, in the case of several processes with the same period.

In general, the HDS-hypothesis supposes the integrating research, where the bifurcation analysis, the control theory, and the climate theory jointly constitute the basis. From the bifurcation analysis, the main evolutionary scenario of a dynamical system with a variable structure represents alternations of several processes with the same periodicity and different structures of phase trajectories during the period. From the control theory, the time and 
amplitude competition described by the $H D S$-law provides the heightened stability of the necessary periodicity on the whole at the expense of regular modifications of phase patterns in particular. From the climate theory, there are several variants of annual temperature patterns, which are clearly connected in temporal-and-temperature sense. Finally, there are typical situations when bifurcations occur but changes of the periodicity are absent (so-called border-collision bifurcations, or, in other words, Cbifurcations); nevertheless, noticeable changes of annual temperature means (and even emergency) can occur with or without such bifurcations.

Taking into account the multidisciplinary circumstances, conclusions were formulated "in comparison" for the uni-behavior and multibehavior viewpoints. In particular, there is the following traditional axiom: a local climate system is characterized by one averaged behavior and deviations from this behavior represent statistical fluctuations determined for the unified 30-year windows. We reformulated that, namely: one of the temperature patterns dominates and other patterns occur to more or less extent depending on the peculiarities of each local climate system, where 30-years represent a dominant duration of evolutionary cycles observed in local climate systems. Alternating qualitative changes (i.e. alternating bifurcations) in local climate dynamics are ordinary events due to the nature of local dynamics. Simply, for the time being, effects of such alternations are quite weak; and, from everyday viewpoint, these effects are neglected (i.e. it is a case of the habitual local climate). However, with some combinations of circumstances, other evolutionary scenarios can appear; in particular, a scenario when abrupt and/or rigid changes of climate patterns appear in various space and time scales.

So, under certain conditions, the traditional uni-behavior viewpoint can be considered as a particular case of the developed multibehavior viewpoint on local climate dynamics based on the $H D S$-hypothesis. Under other conditions, the differences between these viewpoints become too serious. In particular, under the current circumstances, it means that annual temperature variation of each local climate system is described by an ensemble consisting of three elementary processes with 1year periodicity and different patterns (so-called $R$-, $L-, C$-behaviors) encapsulated in the context of the moving temperature hysteresis restricted by local temperature ( $H$-hysteresis, $\left.T_{\text {ref-centerline }}\right)$ and temporal (synchronizing $F_{1}$ - and $F_{2}$-sequences with a half year interval) limits. Reconstruction of such ensemble can be correctly done for a local quasi-homogenous time window determined by a modified bifurcation diagram taking into account change-points determined for local control conditions. This statement first provided a variant of the logically consistent explanation on the interannual variability, building various bifurcation diagrams, where the conceptual model in the form of a dynamical system and factual temperature observations over the last 100-135 years were used for verifications, for example Kolokolov \& Monovskava, 2015a, 2015b, 2015c, 2016a, 2016b, 2016c, 2017a, 2017b, $2017 \mathrm{c}$.

Here, we would like to pay attention to the specific problems of the experimental bifurcation analysis in relation to local climate dynamics. To make it obvious, let us briefly list the conditions which usually occur in relation to a technical system. Then elements and a structure, internal and external parameters, and operating regimes are $a$ priori known. These circumstances allow to form various mathematical models (including conceptual models) with necessary details, where experimental studies aimed at the identification and verification of equivalent parameters have comparatively few restrictions. In particular, it is possible to realize a wide spectrum of physical tests with controlled variation of the parameters and conditions; it is possible to realize accelerated ageing as per various scenarios, including disasters; and so on. Quantity of experimental setups is restricted by financial resources only. So, experimental tests can be concurrently executed with necessary repetitions with practically the same conditions. In other words, collection of the necessary experimental data (i.e. instrumental observations) can be provided over a comparatively short term (several months or several years depending upon a studied system).

What does occur in the case of a local climate system? First, there are a priori unknown constituents which are necessary to form a conceptual model. Each local climate system represents a unique object, in relation to which there are a lot of restrictions on experimental studies in comparison with the abovementioned abilities in relation to a technical system. In particular, there are essential restrictions on the controlled conditions, scenarios, test rate, and so on. Strictly speaking, 
there is only one evolution scenario (the observed scenario), where test rate is restricted by the following rule: one period is equal to one year. Let us compare this with the demands from the experience of the practice-oriented bifurcation analysis aimed at experimentally made identification (i.e. the identification by processing the data of instrumental measurements) of the stability margin for a technical system with a variable structure, for example Kolokolov \& Monovskava, 2013a, 2018]: usually, it is necessary to acquire several tens (and even hundreds) of samples in order to determine one fixed point of mapping for the experimental bifurcation diagram; at least several tens of the fixed points of mapping are necessary to build one realization of the experimental bifurcation diagram; building and processing several tens of such realizations are assumed in order to show the scenario of dynamics evolution with details necessary in practical applications. However, reliable temperature time series on daily means are about $60-140$ years which creates the extreme lack of necessary data. So, it seems to be promising to find a way to get maximum information by processing the existing meteorological data.

We believe that the ideology of the practiceoriented bifurcation analysis seems to be the most promising due to heightened attention to unbiased detailed analysis concerning dissonances between notions connected with natural evolution processes and desirable artificial events. In this context, any climate process is normal to Nature. Atypical climate behaviors represent deviations from human habits and desires. So, if we really would like to comprehend what has happened in climate changes, we should be unprejudiced in estimations. And then, at least the following important moments exist. First, it is a point of the temporal mismatches, for example: a habitual periodicity from human activities (a calendar year, months, seasons, and so on) has approximate relation to the physical essence of the periodicity of a dynamical system under control. Second, a point of the temperature mismatches, for example: averaged temperature characteristics used in traditional estimations on climate changes can represent essentially transformed signals on dynamical processes in local climate systems, where peculiarities of a regularity law and nonlinear properties are not taken into considerations. Third, a point of various opinions concerning climate emergency, where, strictly speaking, two levels exist. The first one relates to practice-oriented opinions on whether an unusual behavior seems to be extremely undesirable or is it just novel reality (Fig. 22)? The second

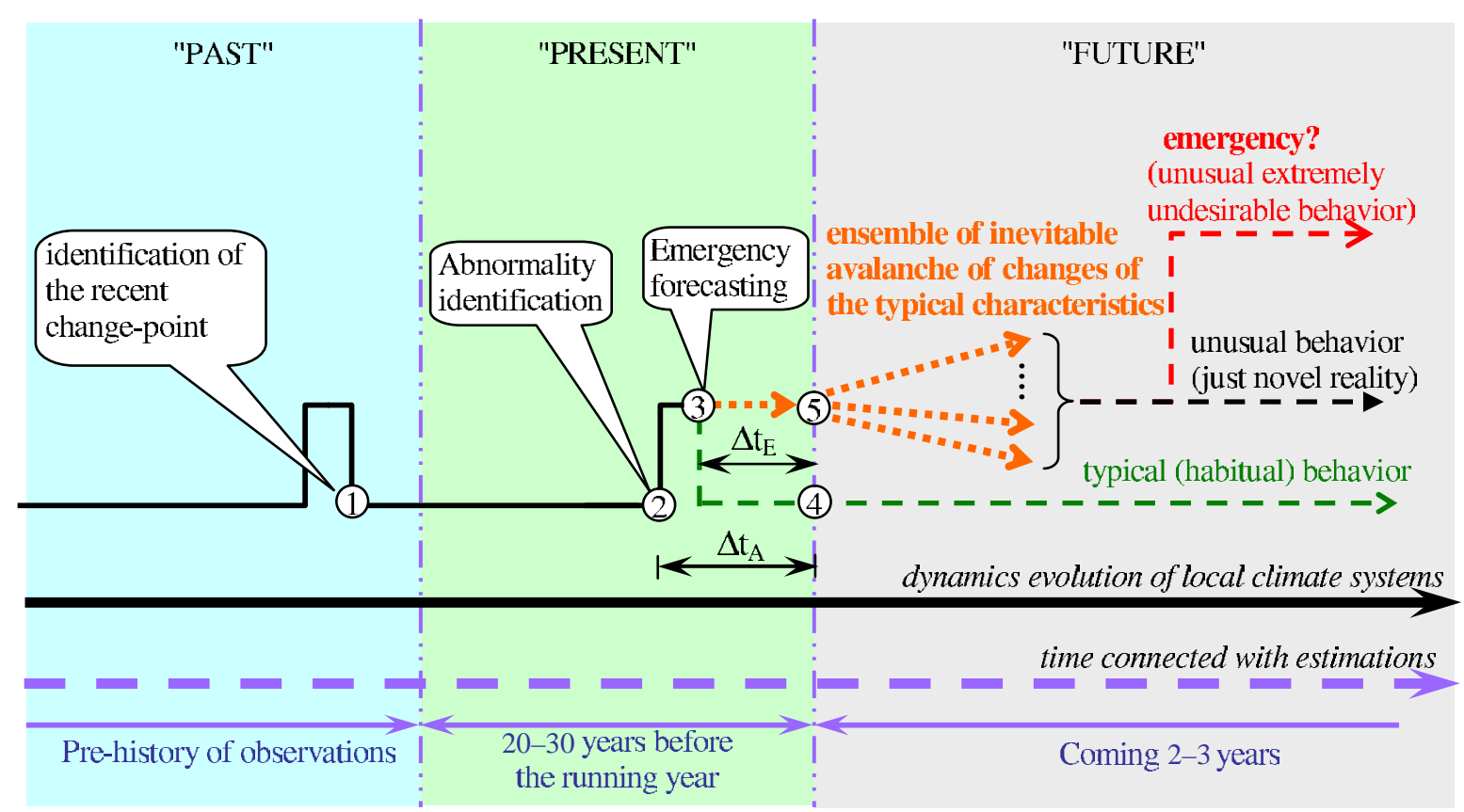

Fig. 22. Scheme to comment on the emergency forecasting in local climate dynamics from the practice-oriented bifurcation analysis, where the objective axis of dynamics evolution is synchronized with the time axis of the subjective terms connected with the existing scientific restrictions. 
relates to variable combinations of economic, social, political, and so on positions which influence these practice-oriented opinions.

Concerning the future outlook, we believe that the integrating idea on emergency forecasting proposed and developed within the practice-oriented bifurcation analvsis. for example [Kolokolov \& Monovskaya, 2018], seems promising in the application to solutions on local climate dynamics as well. Briefly, this idea can be translated by the scheme shown in Fig. 22. Here the abnormalities mirror natural evolutionary processes connected with the nonlinear properties of local climate dynamics [i.e. it is a point of causes in terms of Fig. 20(g)]; these causes inevitably initiate ensemble changes of typical characteristics which can be observed [i.e. it is a point of effects in terms of Fig. [20(g)]. So, once one of these abnormalities is identified, the corresponding ensemble becomes known and it becomes possible to estimate the following: whether the oncoming characteristics lead to emergency [Fig. 20(h)]? We intend to devote the next paper to this topic.

\section{Acknowledgment}

Authors would like to thank reviewers and editors for the support and helpful comments.

\section{References}

Alexander, L. \& Perkins, S. [2013] "Debate heating up over changes in climate variability," Environ. Res. Lett. 8, 041001.

Alisov, B. P. [1956] Climate of the USSR (Moscow University, Moscow) (in Russian).

Argues, A. \& Vose, R. S. [2011] "The definition of the standard WMO climate normal: The key to deriving alternative climate normals," BAMS 92, 699-704.

Bartsev, S., Belolipetskii, P. \& Degermendzhi, A. [2017] "Multistable states in the biosphere-climate system: Towards conceptual models," IOP Conf. Ser.: Mater. Sci. Engin. 173, 012005.

Berge, P., Pomeau, Y. \& Vidal, C. [1988] Order Within Chaos (Wiley, Paris).

Berner, J., Ulrich Achatz, U., Batte, L., Bengtsson, L., de la Camara, A., Christensen, H. M., Colangeli, M., Coleman, D. R. B., Crommelin, D., Dolaptchiev, S. I., Franzke, C. L. E., Friederichs, P., Imkeller, P., Jarvinen, H., Juricke, S., Kitsios, V., Lott, F., Lucarini, V., Mahajan, S., Palmer, T. N., Penland, C., Sakradzija, M., von Storch, J.-S., Weisheimer, A., Weniger, M., Williams, P. D. \& Yano, J.-I. [2017] "Stochastic parameterization: Towards a new view of weather and climate models," BAMS, doi:10.1175/BAMS-D15-00268.1.

Blunden, J. \& Arndt, D. S. (eds.) [2016] "State of the climate in 2015," BAMS 97, S1-S275.

Bondur, V., Krapivin, V. \& Savinikh, V. [2009] Monitoring and Forecasting of the Natural Disasters (Scientific World Publishing House, Moscow) (in Russian).

Budyko, M. I. [1967] The Heat Balance of the Earth's Surface (US Dept. of Commerce, Weather Bureau, Washington).

Bulygina, O. N., Arzhanova, N. M. \& Groisman, P. Y. [2015] "Icing conditions over Northern Eurasia in changing climate," Environ. Res. Lett. 10, 025003.

Buontempo, C., Hewitt, C. D., Doblas-Reyes, F. J. \& Dessai, S. [2014] "Climate service development, delivery and use in Europe at monthly to inter-annual timescales," Clim. Risk Manag. 6, 1-5.

Camus, P., Losada, I. J., Izaguirre, C., Espejo, A., Menendez, M. \& Perez, J. [2017] "Statistical wave climate projections for coastal impact assessments," Earth's Future 5, 918-933.

Cimatoribus, A. A., Drijfhout, S. S. \& Dijkstra, H. A. [2014] "Meridional overturning circulation: Stability and ocean feedbacks in a box model," Clim. Dyn. 42, 311-328.

de Saedeleer, B., Crucifix, M. \& Wieczorek, S. [2013] "Is the astronomical forcing a reliable and unique pacemaker for climate? A conceptual model study," Clim. Dyn. 40, 273-294.

Dijkstra, H. A. [2013] Nonlinear Climate Dynamics (Cambrige Univ. Press, NY).

Dutton, J. A., James, R. P. \& Ross, J. D. [2013] "Calibration and combination of dynamical seasonal forecasts to enhance the value of predicted probabilities for managing risk," Clim. Dyn. 40, 3089-3105.

EFFIS [2018] On-line web page on Long-term seasonal forecast of temperature and rainfall anomalies of European Forest Fire Information System (EFFIS) at the website of the European Commission (http://effis. jrc.ec.europa.eu/applications/seasonal-forecast/).

Eisenman, I. \& Wettlaufer, J. S. [2009] "Nonlinear threshold behavior during the loss of Arctic sea ice," Proc. Natl. Acad. Sci. USA 106, 28-32.

El Aroudi, A., Debbat, M., Giral, R., Olivar, G., Benadero, L. \& Toribio, E. [2005] "Bifurcations in DC-DC switching converters: Review of methods and applications," Int. J. Bifurcation and Chaos 15, 15491578.

Environment and natural resources [2018] On-line web page on Temperature and precipitation probabilistic forecasts of environment and natural resources at the website of the Government of Canada (https:// weather.gc.ca/saisons/prob_e.html).

Essex, C. [2011] "Climate theory versus a theory for climate," Int. J. Bifurcation and Chaos 21, 3477-3487. 
Faghih-Naini, S. \& Shen, B.-W. [2018] "Quasi-periodic orbits in the five-dimensional nondissipative Lorenz model: The role of the extended nonlinear feedback loop," Int. J. Bifurcation and Chaos 28, 1850072-120.

Feigin, M. I. [1978] "On the structure of C-bifurcation boundaries of piecewise continuous systems," J. Appl. Math. Mech. 2, 820-829.

Gallanger, C., Lund, R. \& Robbins, M. [2013] "Changepoint detection in climate time series with long-term trends," J. Clim. 26, 4994-5006.

Glahn, B. [2014] "Determining an optimal decay factor for bias-correcting MOS temperature and dewpoint forecasts," Weather Forecast. 29, 10761090 .

Gockede, M., Kittler, F., Kwon, M. J., Burjack, I., Heimann, M., Kolle, O., Zimov, N. \& Zimov, S. [2017] "Shifted energy fluxes, increased Bowen ratios, and reduced thaw depths linked with drainage-induced changes in permafrost ecosystem structure," The Cryosphere 11, 2975-2996.

Goddard, L., Hurrell, J. W., Kirtman, B. P., Murphy, J., Stockdale, T. \& Vera, C. [2012] "Two time scales for the price of one (almost)," BAMS 93, 621-629.

Grimm, N. B., Staudiger, M. D., Staudt, A., Carter, S. L., Chapin III, F. S., Kareiva, P., Ruckelshaus, M. \& Stein, B. A. [2013a] "Climate-change impacts on ecological systems: Introduction to a US assessment," Front. Ecol. Environ. 11, 456-464.

Grimm, N. B., Chapin III, F. S., Bierwagen, B., Gonzalez, P., Groffman, P. M., Luo, Y., Melton, F., Nadelhoffer, K., Pairis, A., Raymond, P. A., Schimeland, L. \& Williamson, C. E. [2013b] "The impacts of climate change on ecosystem structure and function," Front. Ecol. Environ. 11, 474-482.

Gruza, C. V. \& Rankova, E. Y. [2012] Observed and Expected Climate Change Over Russia: Surface Air Temperature (Institute of Global Climate and Ecology of ROSHYDROMET and Russian Academy of Sciences, Moscow) (in Russian).

Hawkins, E., Ortega, P., Suckling, E., Schurer, A., Hegerl, G., Jones, P., Joshi, M., Osborn, T. J., Masson-Delmotte, V., Mignot, J., Thorne, P. \& van Oldenborgh, G. J. [2016] "Estimating changes in global temperature since the preindustrial period," $B A M S$, doi:10.1175/BAMS-D-16-0007.1.

Hong Kong Observatory [2018] On-line web page on Seasonal forecast for winter 2018/19 at the website of the Hong Kong Observatory (http://www.hko.gov. hk/wxinfo/season/season.htm).

Hourdin, F., Mauritsen, T., Gettelman, A., Golaz, J.-C., Balaji, V., Duan, Q., Folini, D., Ji, D., Klocke, D., Qian, Y., Rauser, F., Rio, C., Tomassini, L., Watanabe, M. \& Williamson, D. [2017] "The art and science of climate model tuning," BAMS 3, 589-602.
Huybers, P., McKinnon, K. A., Rhines, A. \& Tingley, M. [2014] "US daily temperatures: The meaning of extremes in the context of non-normality," J. Clim. 27, 7368-7384.

Hydrometeorological Center of Russia [2018a] The website of the Hydrometeorological Research Center of Russian Federation (www.meteoinfo.ru).

Hydrometeorological Center of Russia [2018b] On-line web page on Seasonal forecast with 1-month lead time at the website of the Hydrometeorological Research Center of Russian Federation (https://meteoinfo.ru/ en/climate/seasonal-forecasts).

Hydrometeorological Center of Russia [2018c] Online web page on Climate of capitals of the world at the website of the Hydrometeorological Research Center of Russian Federation (https:// meteoinfo.ru/en/climate/ climate-for-capitals-of-theworld).

Hydrometeorological Center of Russia [2018d] Online web page on Climate of cities of the world (monthly data) at the website of the Hydrometeorological Research Center of Russian Federation (https://meteoinfo.ru/climatcities).

IPCC Press release [2018] Summary for policymakers of IPCC special report on global warming of $1.5^{\circ} \mathrm{C}$ approved by governments (https://www.ipcc.ch/site/ assets/uploads/2018/11/pr_181008_P48_spm_en.pdf).

Ji, F., Wu, Z., Huang, J. \& Chassignet, E. P. [2014] "Evolution of land surface air temperature trend," Nat. Clim. Change 4, 462-466.

Kattsov, B. M. (ed.) [2017] A Report on Climatic Risk on the Territory of Russian Federation (Voeikov Main Geophysical Observatory of Russia, St. Petersburg) (in Russian).

Katz, R. W., Graidmile, P. F., Guttorp, P., Haran, M., Sanso, B. \& Stein, M. L. [2013] "Uncertainty analysis in climate change assessment," Nat. Clim. Change $\mathbf{3}$, 769-771.

Kim, K.-Y., Hamlington, B. \& Na, H. [2015] "Theoretical foundation of cyclostationary EOF analysis for geophysical and climatic variables: Concepts and examples," Earth-Sci. Rev. 150, 201-218.

Kirtman, B., Power, S. B., Adedoyin, J. A., Boer, G. J., Bojariu, R., Camilloni, I., Doblas-Reyes, F. J., Fiore, A. M., Kimoto, M., Meehl, G. A., Prather, M., Sarr, A., Schar, C., Sutton, R., van Oldenborgh, G. J., Vecchi, G. \& Wang, H. J. [2013] "2013: Near-term climate change: Projections and predictability," in Climate Change 2013: The Physical Science Basis. Contribution of Working Group I to the Fifth Assessment Report of the Intergovernmental Panel on Climate Change, eds. Stocker, T. F., Qin, D., Plattner, G.-K., Tignor, M., Allen, S. K., Boschung, J., Nauels, A., Xia, Y., Bex, V. \& Midgley, P. M., Chapter 11 (Cambridge University Press, Cambridge), pp. 953-1028. 
Klimenko, V. V., Fedotova, E. V. \& Tereshin, A. G. [2018] "Vulnerability of the Russian power industry to the climate change," Energy 142, 1010-1022.

Kolokolov, Yu., Kukin, A. \& Zhusubaliev, Zh. [1991] "Author's certificate SU 1533904. Method of DC drive current control," (Priority date 14.01.1987, Publication date 15.03.1991) Bull. Invent. USSR 10 (in Russian).

Kolokolov, Yu. V., Koschinsky, S. L., Hatziadoniu, C. \& Galanos, G. [2003] "Dynamics of current regulator with hysteresis control and clocked commutation in application to power electronic systems," IEEE Int. Conf. Physics and Control, pp. 1165-1169.

Kolokolov, Yu. \& Monovskaya, A. [2006] "Modified bifurcation diagrams in an approach to on-line pulse system dynamics forecasting," Int. J. Bifurcation and Chaos 16, 85-100.

Kolokolov, Yu. \& Monovskaya, A. [2013a] "From modifications of experimental bifurcation diagrams to operating process stability margin," Int. J. Bifurcation and Chaos 23, 1330024-1-20.

Kolokolov, Yu. \& Monovskaya, A. [2013b] "Fractal approach, bifurcation poker and SUC-logic for nonlinear dynamics forecasting," Int. J. Bifurcation and Chaos 23, 1350201-1-18.

Kolokolov, Yu. \& Monovskaya, A. [2015a] "Guess-work and reasonings on centennial evolution of surface air temperature in Russia: Is it possible to build bifurcation diagrams based on extra-short local observations?" Int. J. Bifurcation and Chaos 25, 1550084-124.

Kolokolov, Yu. \& Monovskaya, A. [2015b] "Modified bifurcation diagrams to analyse the intermittency observed in local climate dynamics," IEEE Int. Conf. Intelligent Data Acquisition and Advanced Computing Systems: Technology and Applications, pp. 624-630.

Kolokolov, Yu. \& Monovskaya, A. [2015c] "Estimating of temperature abnormalities in local climate dynamics," IEEE Int. Conf. Intelligent Data Acquisition and Advanced Computing Systems: Technology and Applications, pp. 598-604.

Kolokolov, Yu. \& Monovskaya, A. [2015d] "Multidimensional analysis of dynamics of annual warmingcooling cycles on the basis of index model of temperature observations," IEEE Int. Conf. Intelligent Data Acquisition and Advanced Computing Systems: Technology and Applications, pp. 631-637.

Kolokolov, Yu. \& Monovskaya, A. [2015e] "Mechanism of latent abnormalities in dynamics of hysteresis regulator with double synchronization," IEEE Int. Conf. Intelligent Data Acquisition and Advanced Computing Systems: Technology and Applications, pp. 587592.

Kolokolov, Yu. \& Monovskaya, A. [2015f] "Startup peculiarities in dynamics of hysteresis regulator with double synchronization," IFAC-PapersOnline 48, 964-969.

Kolokolov, Yu. \& Monovskaya, A. [2016a] "Guess-work and reasonings on centennial evolution of surface air temperature in Russia. Part II: Is it possible to research both local peculiarities and regional tendencies from the bifurcation analysis viewpoint?" Int. J. Bifurcation and Chaos 26, 1650071-1-13.

Kolokolov, Yu. \& Monovskaya, A. [2016b] "Guess-work and reasonings on centennial evolution of surface air temperature in Russia. Part III: Where is the joint between norms and hazards from the bifurcation analysis viewpoint?" Int. J. Bifurcation and Chaos 26, 1650122-1-20.

Kolokolov, Yu. \& Monovskaya, A. [2016c] "Guess-work and reasonings on centennial evolution of surface air temperature in Russia. Part IV: Towards economic estimations of climate-related damages from the bifurcation analysis viewpoint," Int. J. Bifurcation and Chaos 26, 1630033-1-17.

Kolokolov, Yu. \& Monovskaya, A. [2017a] "Observationsbased computational analytics on local climate dynamics: Change-points," Int. J. Computing 16, 89-96.

Kolokolov, Yu. \& Monovskaya, A. [2017b] "Observations-based computational analytics on local climate dynamics. Part 2: Seasonality," Int. J. Computing 16, 152-159.

Kolokolov, Yu. \& Monovskaya, A. [2017c] "Observationsbased computational analytics on local climate dynamics. Part 3: Forecasting," Int. J. Computing 16, 210-218.

Kolokolov, Yu. \& Monovskaya, A. [2018] "A practiceoriented bifurcation analysis for pulse energy converters. Part 4: Emergency forecasting," Int. J. Bifurcation and Chaos 28, 1850152-1-27.

Kose, N., Guner, H. T., Harley, G. L. \& Guiot, J. [2017] "Spring temperature variability over Turkey since 1800CE reconstructed from a broad network of tree-ring data," Climate of the Past 13, 1-15.

Krauskopf, B. \& Sieber, J. [2014] "Bifurcation analysis of delay-induced resonances of the El-Nino Southern Oscillations," Proc. Roy. Soc. A 470, 20140348.

Lorenz, E. N. [1963] "Deterministic nonperiod flow," J. Atmos. Sci. 20, 130-141.

Lucarini, V. \& Bodai, T. [2017] "Edge states in the climate system: Exploring global instabilities and critical transitions," Nonlinearity 30, R32-R66.

Mantua, N. J., Hare, S. R., Zhang, Y., Wallace, J. M. \& Francis, R. C. [1997] "A Pacific decadal climate oscillation with impacts on salmon," BAMS 78, 10691079.

Margaritelli, G., Cisneros, M., Cacho, I., Capotondi, L., Vallefuoco, M., Rettori, R. \& Lirer, F. [2018] "Climatic variability over the last 3000 years in the 
central-western mediterranean sea (Menorca Basin) detected by planktonic foraminifera and stable isotope records," Global and Planetary Change 169, 179-187.

Mayewski, P. A., Carleton, A. M., Birkel, S. D., Dixon, D., Kurbatov, A. V., Korotkikh, E., Mc Connell, J., Curran, M., Cole-Dai, J., Jiang, S., Plummer, C., Vance, T., Maasch, K. A., Sneed, S. B. \& Handley, M. [2017] "Ice core and climate reanalysis analogs to predict Antarctic and Southern Hemisphere climate changes," Quater. Sci. Rev. 155, 20-66.

MGO [2018] On-line web page on Climate changes in Russia in the 21-st century (CMIP5) at the website of the Voeikov Main Geophysical Observatory of Russia (MGO), St. Petersburg (http://voeikovmgo. $\mathrm{ru} /$ index.php?option $=$ com_content\&view $=$ article\&id $=613 \&$ Itemid $=236 \&$ lang $=\mathrm{ru}$ ).

NOAA [2018] On-line web page on Experimental unofficial two-class monthly and seasonal climate outlooks of the Climate Prediction Center of the U.S. National Weather Service at the website of National Oceanic and Atmospheric Administration (NOAA) (http://www.cpc.ncep.noaa.gov/products/predictions/ long_range/two_class.php).

NOAA Fisheries [2018] On-line web page on Pacific Decadal Oscillation of Northwest Fisheries Science Center at the website of National Oceanic and Atmospheric Administration (NOAA) (http://www.nwfsc. noaa.gov/research/divisions/fe/estuarine/oeip/ca-pdo. cfm).

Novak, D. R., Bailey, C., Brill, K. F., Burke, P., Hogsett, W. A., Rausch, R. \& Schichtel, M. [2014] "Precipitation and temperature forecast performance at the weather prediction center," Weather and Forecasting 29, 489-504.

Oh, J., Reischmann, E. \& Rial, J. A. [2014] "Polar synchronization and the synchronized climatic history of Greenland and Antarctica," Quater. Sci. Rev. 83, 129-142.

Palmer, T. N. [2014] "More reliable forecasts with less precise computations: A fast-track route to cloudresolve weather and climate simulators?" Philos. Trans. Roy. Soc. A 372, 20130391.

Palmer, T. N., Doring, A. \& Seregin, G. [2014] "The real butterfly effect?" Nonlinearity 27, R123-R141.

Pezzulli, S., Stephenson, D. B. \& Hannachi, A. [2005] "The variability of seasonality," J. Clim. 18, $71-88$.

Rial, J. A., Pielke Sr., R. A., Beniston, M., Claussen, M., Canadell, J., Cox, P., Held, H., de NobletDucoudre, N., Prinn, R., Reynolds, J. F. \& Salas, J. D. [2004] "Nonlinearities, feedbacks and critical thresholds within the Earth's climate system," Clim. Change 65, 11-38.
RIHMI-WDC [2018a] The website of All-Russian Research Institute of Hydrometeorological Information - World Data Center (RIHMI-WDC) (www.meteo.ru).

RIHMI-WDC [2018b] On-line web page on Climate conditions over Russia at the website of All-Russian Research Institute of Hydrometeorological Information - World Data Center (RIHMI-WDC) (http:// meteo.ru/pogoda-i-klimate/93-klimaticheskie-usloviya/ 179-klimaticheskie-usloviya-na-territorii-rossii).

RT news [2018] (https://www.rt.com/business/423913northern-sea-route-us/).

Powers, J. G., Klemp, J. P., Skamarock, W. C., Davis, C. A., Dudhia, J., Gill, D. O., Coen, J. L., Gochis, D. J., Ahmadov, R., Peckham, S. E., Grell, G. A., Michalakes, J., Trahan, S., Benjamin, S. J., Alexander, C. R., Dimego, G. J., Wang, W., Schwartz, C. S., Romine, G. S., Liu, Z., Snyder, C., Chen, F., Barlage, M. J., Yu, W. \& Duda, M. G. [2017] "The weather research and forecasting model. Overview, system efforts, and future directions," BAMS $\mathbf{8}, 1717-$ 1737.

Rueda, A., Vitousek, S., Camus, P., Tomas, A., Espejo, A., Losada, I. J., Barnard, P. L., Erikson, L. H., Ruggiero, P., Reguero, B. G. \& Mendez, F. J. [2017] "A global classification of coastal flood hazard climates associated with large-scale oceanographic forcing," Sci. Rep. 7, 5038, doi:10.1038/s41598-017-05090-w.

Ruff, T. W. \& Neelin, J. D. [2012] "Long tails in regional surface temperature probability distributions with implications for extremes under global warming," Geophys. Res. Lett. 39, L04704.

Scheffer, M., Bascompte, J., Brock, W. A., Brovkin, V., Carpenter, S. R., Dacos, V., Held, H., van Nes, E. H., Rietkerk, M. \& Sugihara, G. [2009] "Early-warning signals for critical transitions. Reviews," Nature 461, $53-59$.

Sevellec, F. \& Drijfhout, S. S. [2018] "A novel probabilistic forecast system predicting anomalously warm 2018-2022 reinforcing the long-term global warming trend," Nat. Commun. 9, 3024, doi: 10.1038/s41467018-05442-8.

Sillmann, J., Russo, S., Sippel, S. \& Alnes, K. [2018] "From hazard to risk," BAMS 8, 1689-1693.

Sippel, S., Zscheischler, J., Heimann, M., Otto, F. E. L., Peters, J. \& Mahecha, M. D. [2015] "Quantifying changes in climate variability and extremes: Pitfalls and their overcoming," Geophys. Res. Lett. 42, 99909998.

Sippel, S., Reichstein, M., Ma, X., Mahecha, M. D., Lange, H., Flach, M. \& Frank, D. [2018] "Drought, heat, and the carbon cycle: A review," Curr. Clim. Change Rep., https://doi.org/10.1007/s40641-018-0103-4. 
Soares, M. B. \& Dessai, S. [2015] "Exploring the use of seasonal climate forecasts in Europe through expert elicitation," Clim. Risk Assess. 10, 8-16.

Song, H., Liu, Y., Li, Q., Gao, N., Ma, Y. \& Zhang, Y. [2014] "Tree-ring based May-July temperature reconstruction since AD 1630 on the Western loess plateau, China," PLoS ONE 9, e93504, doi: 10.1371/journal.pone.0093504.

Sooraksa, P. \& Chen, G. [2018] "Chen system as a controlled weather model — Physical principles, engineering design and real applications," Int. J. Bifurcation and Chaos 28, 1830009-1-12.

Stephens, G. L., Li, J., Wild, M., Clayson, C. A., Loeb, N., Kato, S., L'Ecuyeer, T., Stackhous Jr., P. W., Lebsock, M. \& Andrews, T. [2012] "An update on Earth's energy balance in light of the latest global observations," Nat. Geosci. 5, 691-696.

Stine, A. S. \& Huybers, P. [2012] "Changes in the seasonal cycle of temperature and atmospheric circulation," J. Clim. 25, 7362-7380.

Stocker, T. F. et al. (eds.) [2013] "Summary for Policymakers," Climate Change 2013: The Physical Science Basis. Contribution of Working Group I to the Fifth Assessment Report of the Intergovernmental Panel on Climate Change (Cambridge Univercity Press, Cambridge), Chapter SPM, pp. 3-29.

Sundqvist, H. S., Kaufman, D. S., McKay, N. P., Balascio, N. L., Briner, J. P., Cwynar, L. C., Sejrup, H. P., Seppa, H., Subetto, D. A., Andrews, J. T., Axford, Y., Bakke, J., Birks, H. J. B., Brooks, S. J., de Vernal, A., Jennings, A. E., Ljungqvist, F. C., Ruhland, K. M., Saenger, C., Smo, J. P. \& Viau, A. E. [2014] "Arctic Holocene proxy climate database - New approaches to assessing geochronological accuracy and encoding climate variables," Climate of the Past 10, 1605-1631.

Thompson, J. M. \& Sieber, J. [2011] "Predicting climate tipping as a noisy bifurcation: A review," Int. J. Bifurcation and Chaos 21, 399-423.

Tol, R. S. J. [2016] "The impacts of climate change according to the IPCC," Clim. Change Econ. 7, 1640004.

Trevisan, A. \& Palatella, L. [2011] "Chaos and weather forecasting: The role of the unstable subspace in predictability and state estimation problems," Int. J. Bifurcation and Chaos 21, 3389-3415.

Tse, C. K. [2004] Complex Behavior of Switching Power Converters (CRC Press, Boca Raton, FL).
Uboldi, F. \& Trevisan, A. [2015] "Multi-scale error growth in a convection-resolving model," Nonlin. Process. Geophys. 22, 1-13.

von Schuckmann, K., Palmer, M. D., Trenberth, K. E., Cazenave, A., Chambers, D., Champollion, N., Hansen, J., Josey, S. A., Loeb, N., Mathieu, P.-P., Meyssignac, B. \& Wild, M. [2012] "An imperative to monitoring Earth's energy imbalance," Nat. Clim. Change 6, doi: 10.1038/NCLMATE2876.

Westervelt, D. M., Horowitz, L. W., Naik, V., Golaz, J.-C. \& Mauzerall, D. L. [2015] "Radiative forcing and climate response to projected 21st century aerosol decreases," Atmos. Chem. Phys. 15, 12681-12703.

Williamson, M. S., Bathiany, S. \& Lenton, T. M. [2016] "Early warming signals of tipping points in periodically forced systems," Earth Syst. Dyn. 7, 313-326.

WMO-No.1137 [2014] Documents of the 16th Session of the Commission for Climatology, Heidelberg, Germany, 3-8 July 2014: CCL-16/Doc.8.1. Final Report with Resolutions and Recommendations (World Meteorological Organization, Geneva).

WMO-Bulletin 66(2) [2017] Weather Ready, Climate Smart - Supporting the 2030 Agenda for Sustainable Development (World Meteorological Organization, Geneva).

WMO-No.1212 [2018] Statement on the State of the Global Climate in 2017 (World Meteorological Organization, Geneva).

Yakovenko, M. E. (ed.) [2018] A Report on Climate Features on the Territory of the Russian Federation in 2017 (Russian Federal Service for Hydrometeorology and Environmental Monitoring, Moscow) (in Russian).

Yin, J., Gentine, P., Zhou, S., Sullivan, S. C., Wang, R., Zhang, Y. \& Guo, S. [2018] "Large increase in global storm runoff extremes driven by climate and anthropogenic changes," Nat. Commun. 9, 4389, doi:10.1038/s41467-018-06765-2.

Zaytsev, A. P., Podlyagin, V. A., Kolokolov, Yu. V., Veitsman, L. Yu. \& Barsky, M. P. [1975] "Author's certificate SU 481476: Hysteresis regulator of electric motor current," (Priority date 27.12.1973, Publication date 25.08.1975) Bull. Invent. USSR 31 (in Russian).

Zhou, G., Xu, J. \& Bao, B. [2012] "Symmetrical dynamics of current-mode controlled switching DC-DC converters," Int. J. Bifurcation and Chaos 22, 1250008$1-11$. 